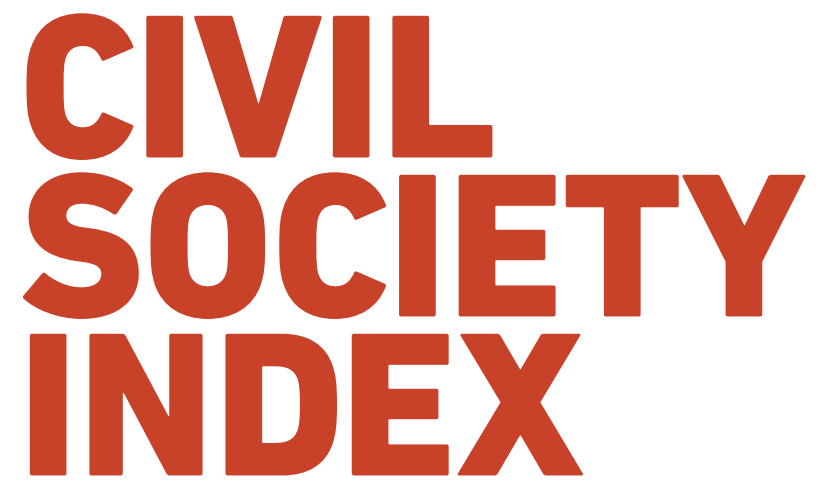

Rapid Assessment

RAHMENBEDINGUNGEN FÜR DIE

ZIVILGESELLSCHAFT IN ÖSTERREICH

Eva More-Hollerweger, Ruth Simsa, Günther Kainz, Franz Neunteufl, Romy Grasgruber-Kerl,

Felix Sebastian Wohlgemuth

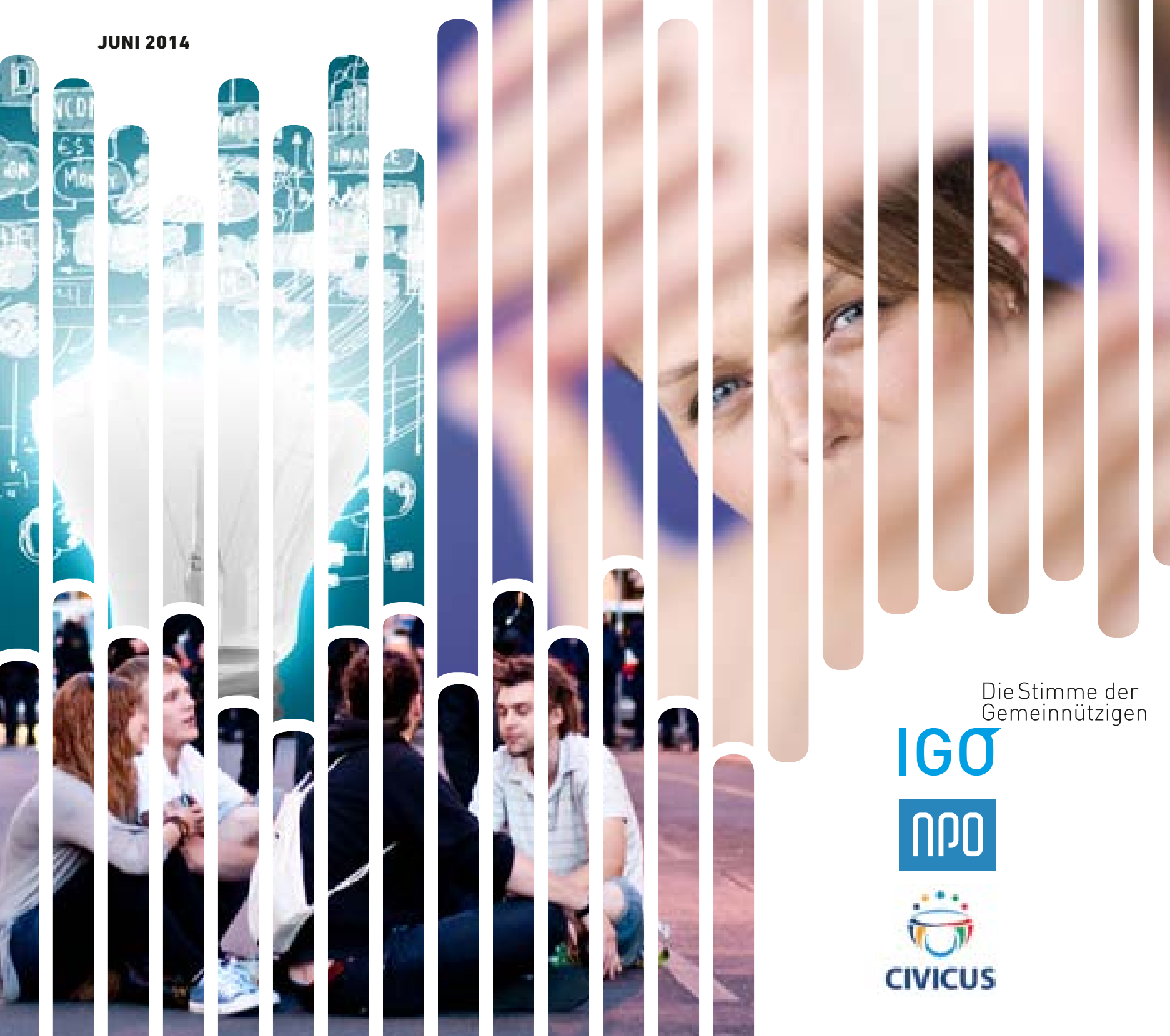




\section{CIVIL SOCIETY INDEX}

Rapid Assessment

RAHMENBEDINGUNGEN

FÜR DIE

ZIVILGESELLSCHAFT

IN OSTERREICH

Eva More-Hollerweger, Ruth Simsa,

Günther Kainz, Franz Neunteufl,

Romy Grasgruber-Kerl,

Felix Sebastian Wohlgemuth

JUNI 2014 


\section{INHALT}

\section{MPRESSUM:}

ht ist in Kooperation zwischen

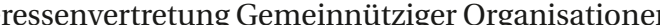

Kompetenzzentrum für Nonprofit Organisationen und Social Entrepreneurship und CIVICUS - World Alliance for Citizen Participation entstanden.

\section{IGO}

nteressenvertretung Gemeinnütziger Organisationen

Stubenring $2 / 4$

IоIо Wien

www.gemeinnuetzig.at

Kontakt: office@gemeinnuetzig.at

\section{กก $\mathbf{W} \boldsymbol{U}_{\overline{\mathrm{I}}}$}

Kompetenzzentrum für Nonprofit Organisationen

und Social Entrepreneurship

Wirtschaftsuniversitât

Welthandelsplatz

ro20 Wien

Kontakt: eva.more@wu.ac.at

\section{(*) \\ civicus}

COVIld Alliance for Citizen Participation

PO BOX 933, Southdale 2135, JHB, South Africa

Kontakt: patricia.deniz@civicus.or

Copyright $\odot$ NPO \&SE Kompetenzzentrum,

Interessenvertretung Gemeinnütziger Organisationen

Gestaltung: Peter Kratzer $\cdot$ www.co-des.at

Print CO2 geprüft / ISO I400I Zertifizierung / Osterreichisches Umweltzeichen /

PEFC Zertizierung 06-39-17/ Qualtatsmanagenent-System IsO goor:2001/Prozess

Standard Offsetdruck ISO I2647-2 / FSC-Zertifizierung Ci08222 / Konformitätserklärung 


\section{Vorwort}

— ine lebendige Zivilgesellschaft ist wichtig für gelebte Demokratie, für sozialen Zusammenhalt und für gesellschaftliche Innovationen. Zivilgesellschaftliche Organisationen bieten darüber hinaus wichtige Dienstleistungen an. Insbesondere in Krisituation vieler Menschen dienen.

Damit die Potenziale der Zivilgesellschaf moglichst gut entfaltet und genutzt werden kônnen, braucht es geeignete Rahmenbedingungen. Der Forschung ist dies schon lange bekannt und auch die Politik entdeckt zunehmend die Chancen, die in der Schaffung von guten Bedingungen für die Zivilgesellschaft liegen.

Index - Rapid Assessment (CSI-RA) wurde daher untersucht, wie es um das Klima und die Rahmenbedingungen für zivilgesellschaftliche Initiativen und Organisationen in Osterreich bestellt ist. Förderliche wie auch hemmende Faktoren wurden aus der Sicht von Exper-

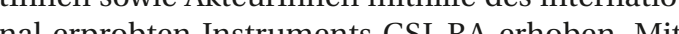

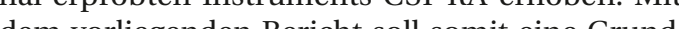

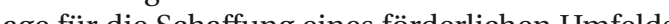
geboten werden.

\section{Danksagung}

eser Bericht ist in einer Kooperation zwischen der Interessenvertretung Gemeinnütziger Organisationen (IGO) und dem NPO Kompetenzzentrum für Nonprofit Organisationen und Social Entrepreneurship entstanden. Die Methode wurde von CIVICUS World Alliance for Citizen Participation entwickelt.

Der Civil Society Index - Rapid Assessmen wurde unter Mitwirkung einer großen Anzahl von Menschen realisiert, bei denen wir uns an dieser Stelle herzlich bedanken môchten. Unser Dank geh an die Mitglieder der National Advisory Group für deren Mitwirkung bei der thematischen Weichenstellung und Interpretation der Studienergebnisse. Es handelt sich dabei um Karin Abram, Rober Dempfer, Nikolaus Dimmel, Gerald Fitz, Gabriele Gerbasits, Katharina Meichenitsch, Filip Radunovic Hanna Simons, Philipp Sonderegger, Babara Webe und Winhelm Zwirner.

Außerdem danken wir den mehr als 20 Interwewparte in sungen fir die Abit gesellschaft ermöglichten.$$
\text { Weiters bedanken wir }
$$

wir uns bei Katrin Hora, Klinghofer für die Mitarbei beim Führen und Transkribieren der Interviews. Gottfried Sulz, Andreas Lummerstorfer und Philipp Hense danken wir für ihre Expertise aus Theorie und Praxis, die uns bei der Finalisierung des Berichts sehr geholfen hat

Last but not least möchten wir jenen Institutionen danken, durch deren Unterstützung dieses für Österreich einzigartige Projekt erst möglich wurde, nämlich der RD Foundation, der Erste Stiftung, der Erste Bank und der Allianz Gruppe in Österreich.

Eva More-Hollerweger, Romy Grasgruber-Kerl,

Franz Neunteufl und Ruth Simsa (National Implementation Coalition)

\begin{tabular}{|c|c|}
\hline ABKURL & GSVERLEICHNIS \\
\hline AGBG & $\begin{array}{l}\text { Allgemeines Bürgerliches } \\
\text { Gesetzbuch }\end{array}$ \\
\hline$\overline{\mathrm{BAO}}$ & Bundesabgabenordnung \\
\hline BVergG & Bundesvergabegesetz \\
\hline$\overline{B W G}$ & Bankwesengesetz \\
\hline BZÖ & Bündnis Zukunft Österreich \\
\hline CSI & Civil Society Index \\
\hline CSI-RA & $\begin{array}{l}\text { Civil Society Index- } \\
\text { Rapid Assessment }\end{array}$ \\
\hline CSO & Civil Society Organisation \\
\hline ECNL & $\begin{array}{l}\text { European Center for } \\
\text { Non-Profit Law }\end{array}$ \\
\hline EMRK & $\begin{array}{l}\text { Europäischen Menschenrechts } \\
\text { konvention }\end{array}$ \\
\hline$\overline{\mathrm{EStG}}$ & Einkommensteuergesetz \\
\hline FPÖ & Freiheitliche Partei Österreichs \\
\hline GmbH & $\begin{array}{l}\text { Gesellschaft mit beschränkter } \\
\text { Haftung }\end{array}$ \\
\hline IGO & $\begin{array}{l}\text { Interessenvertretung } \\
\text { Gemeinnütziger Organisatione }\end{array}$ \\
\hline
\end{tabular}

\begin{tabular}{ll} 
KESt & Kapitalertragsteuer \\
\hline KommStG & Kommunalsteuergesetz \\
\hline KStG & Körperschaftsteuergesetz \\
\hline NAG & National Advisory Group \\
\hline NGO & Non Governmental Organisation \\
\hline NPO & Nonprofit Organisation \\
\hline ORF & Österreichische Rundfunk \\
\hline ORF-G & $\begin{array}{l}\text { Bundesgesetz über den } \\
\text { Österreichischen Rundfunk }\end{array}$ \\
\hline ÖVP & Österreichische Volkspartei \\
\hline PSG & Privatstiftungsgesetz \\
\hline SPÖ & Sozialdemokratische Partei \\
\hline SPG & Sicherheitspolizeigesetz \\
\hline SROI & Social Return on Investment \\
\hline StGB & Strafgesetzbuch \\
\hline StVO & Straßenverkehrsordnung \\
\hline UGB & Unternehmenssgesetzbuch \\
\hline VerG & Vereinsgesetz \\
\hline VersG & Versammlungsgesetz \\
\hline VGT & Verein gegen Tierfabriken
\end{tabular}

Abbildung I: Schwerpunktthemen der Studie

Abbildung 2: Democracy-Barometer: demokratische Qualität Österreichs

Abbildung 4: Wissensstand zu gemeinnützigen bzw. Non-Profit-Organisationen

Abbildung 5: Gesellschaftlicher Stellenwert von NPOs

Abbildung 6: Wahrnehmung möglicher zukünftiger Unterstützung für NPO 
Demokratie in Österreich ist der erste gewählte Themenbereich der Analyse. Die demokratische Qualität wird anhand des Democracy Barometers dargestellt, das 30 etablierte Demokratien entlang der drei Dimensionen Freiheit, Gleichheit und Kontrolle sowie je drei weiterer Subdimensionen vergleicht. Die Stärken der österreichischen Demokratie liegen in der Garantie und der Auslebung de individuellen Freiheiten sowie in der Rechtsstaatlichkeit. Die Partizipation ist hingegen nur durchschnittlich ausgeprägt. Vergleichsweise schlechte Noten erhält Österreich Bezug auf die öffentlich Sphäre, was vor allem durch die hohe Medienkonentration bedingt ist.

Das Themenfeld BürgerInnen- und Men schenrechte umfasst drei Subthemen, die Vereinigungsfreiheit, die Versammlungsfreiheit und den Zugang von CSOs zu finanziellen Ressourcen. Die Vereinigungsfreiheit ist in Österreich rechtlich gut verankert und wird in der Praxis auch vielfach umge setzt. Schwierigkeiten gab es in jüngerer Zeit vor allem in Verbindung mit dem Gesinnungsstrafrecht. Tierschüzerinnen wurde vorgeworfen, sich an de S 278a StGB beteiligt zu Oaben. Auch in Bezue des die Vers bezug au die Versammlungsfreiheit stellen die rechtlichen Bestimnungen eine gute Basis dar. Eine explizite dernisse der Europäischen Menschenrechtskonvention wäre jedoch notwendig, um mehr Klarheit in den Bereich zu bringen. Versammlungen sind in Österreich nicht bewilligungspflichtig, bedürfen jedoch einer Nichtuntersagung. Kritisch beurteilt wurde vor allem die Umsetzung der rechtlichen Regelungen in Bezug auf Untersagungen und das Verhalten der Exekutive bei Versammlungen. Der Zugang zu finanziellen Ressourcen ist für CSOs in Österreich weitgehend gewährleistet. Einschränkungen müssen CSOs dann in Kauf nehmen, wenn sie den Status der Gemeinnützigkeit erhalten wollen da dieser mit Steuererleichterungen verbunden ist. Eine österreichische Besonderheit stellt das Privatstiftungsrecht dar, das bislang wenig auf Gemeinnützigkeit ausgerichtet ist und eher eigennützige Privatstiftungen fördert. Entsprechend gering ist die Zahl der gemeinnützigen Stiftungen, wodurch auch eine in anderen Ländern wichtige Finanzierungsquelle für CSOs wegfällt. Derzeit gibt es Bestrebungen, ein eigenes Gesetz für gemeinnützige Privatstif-
tungen zu etablieren.

Fragen des Steuerrechts werden im Rahmen des dritten Themenbereichs erlautert, wobei hie der Schwerpunter dengesež̈lcherich liegt. Ih Osten und Steuervorteilen für remeinnë von mildtätige und kirchliche CSOs, bei deren untzige mildtatige und kircht es aber nur bedingte Rechtssicherbeit. dung gibt es abor nur bedingte Rechtssicherheit. deren teilweise willkürliche Interpretation durch die Finanzbehörden bei wirtschaftlichen Geschäftsbetrieben. Dem gegenüber steht die teilweise sehr rigorose Anwendung von formalen Anforderungen die, so sie in den Augen der Behörde nicht erfüllt sind, rückwirkend zum Verlust der steuerlichen Begünstigung führen und für die CSO zur Existenzbedrohung werden können.
Beschäftigung und Freiwilligenarbeit bilden Informationen über infrage kommende zukünftige den vierten Themenschwerpunkt, mit dem sich die Förderungen zu geben und den Einreichprozess zu Analyse befasst. Besonderes Merkmal von CSOs sind die vielfältigen Formen, in denen Menschen für CSOs tätig sind. Neben Erwerbstätigen sind das vor allem Freiwillige. Seit 2012 gibt es in Österreich ein Freiwilligengesetz, das verschiedene Formen des freiwilligen Engagements regelt. Dennoch bleibt ein gewisser Graubereich, für den wenig Rechtssicherheit besteht, beispielsweise bei mehrmonatigen freiwilligen Einsatzen im Ausland. Im Hinblick auf die Förderung des freiwilligen Engagements gibt es eine breite Palette an Verbesserungsvorschlagen, beispielsweise in Hinblick auf deren Versicherungsschutz (insbesondere Haftpflicht- und Unfallversicherung.

Der fünfte Themenbereich nimmt das Verhältnis zwischen öffentlicher Hand und CSOs in Bezug auf die Bereitstellung von Dienstleistungen ins Visier. CSOs stellen in Osterreich eine Vielzahl
an Dienstleistungen bereit, vielfach auch dadurch an Dienstleistungen bereit, vielfach auch dadurch bedingt, dass der Staat vormals selbst angebotene
Dienstleistung zunehmend an private Organisationen auslagert Die öffentliche Hand übernimmt zwar in weien Tilen die Finanzerch die Rabmenbedingungen daür lich. Grundoützlich kann die Finanzierung einerseits über Leistungsverträge und andererseits durch Förderungen erfolgen, wobei die Unterschiede in der Ausgestaltung derVerträge der beiden Finanzierungsformen fließend sind. Entscheidend ist dass für Leistungsverträge das Vergaberecht zum Tragen kommt, das im Wesentlichen darauf ausgerichtet ist, den Wettbewerb zu stärken. Problematisch ist, dass CSO hierbei oft mit gewinnorientierten Anbietern in Konkurrenz treten, die sich in ihren Zielsetzungen und in ihrer Art der Produktion sehr stark unterscheiden. CSOs erzeugen ihrem ideellen Zweck entsprechend haufig eine Vielfalt an positiven externen Effekten, die beim Vergabeverfahren jedoch nicht zum Tragen kommen. Diese Elekte entstehen beispielsweise durch den Einsatz von Freiwilligen oder durch die Erfüllung der Funktion der Interessenvertretung für Zielgruppen, für die sie auch Dienstleistungen erbringen. Bestrebungen gehen dahin, die Gemeinnützigkeit im Vergaberecht stärkerzu verankern, um gesellschaftlichen Zielen einen höheren Stellenwert zu geben. Bei Förderungen sind die Rahmenbedingungen für CSOs oft ebenfalls sehr schwierig. Zu den Herausforderungen zahlen die fehlende Indexanpassung, Einschrânchen rehende Han der öfentlichen Hand, sowie späte Zahlungen. Dies führt zu hohen ökonomischen Risiken bei CSOs, die vor allem für kleine CSOs oft kaum tragbar sind Neben der allgemeinen Beschreibung der Nusgestaltung der Beziehung zwischen öffentlicher Hand und CSOs wird der Transparenz ein eigenes Kapitel gewidmet. In den letzten Jahren gab es einige Bemühungen, die Transparenz bei derVergabe von Leistungsverträgen und Förderungen zu erhöhen, ein Beispiel dafür ist die - noch laufende - stufenweise Erstellung eines Transparenzportals, mit dem Ziel, eine einheitliche Darstellung aller bundesweit angebotenen Förderleistungen zu schaffen, nterstützen. Mehr Transparenz wurde seitens der

Der letzte Themenbereich umfasst die öffentliche Wahrnehmung zivilgesellschaftlicher Wirkungen. Dafür wurden in einer repräsentativen Telefonumfrage Wissensstand, Wahrnehmung und Einschătzung von gemeinnützigen bzw. Non-Profit-Organisationen in Osterreich untersucht. 70 Prozent der osterreichischen Bevölkerung schätzen sich diesbeztglich als gut informiert ein. 9 von ro Befragten schreiben den Organisationen einen hohen Stel. nung, dass die Bedeutung von NPOs in Zukunft 列 then hatten in irgendeiner Form bereits personnli-

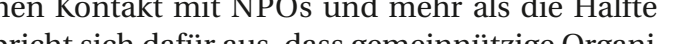
pationen durch direkte Förderungen oder Steganileichterungen stärker unterstützt werden. InterviewpartnerInnen in Bezug auf die Ablehnun on Anträgen bzw. Angeboten eingefordert. 


\section{AUFBAU DER ARBEIT}

er Schwerpunkt der vorliegenden Studie wurde Kapitel I beleuchtet den Projekthintergrund und unter Einbeziehung erfahrener AkteurInnen der beschreibt die methodische Herangehensweise, die Zivilgesellschaft auf die rechtlichen Rahmenbedin- im Rahmen des Civil Society Index - Rapid Assess-
gungen für zivilgesellschaftliches Engagement in
ment (CSI-RA) gewählt und umgesetzt wurde. Die gungen fur zivilgesellschaftliches Engagement in ment (CSI-RA) gewahlt und umgesetzt wurde. Die
Österreich sowie auf die Wahrnehmbarkeit zivilge- Ausführungen enthalten auch eine kurze Erläutesellschaftlicher Wirkungen gelegt. Die Themenbe- rung der Grenzen dieser Herangehensweise. reiche betreffen eine große Vielfalt an Organisatio-

nen und Initiativen, die in unterschiedlicher Weise Kapitel II gibt einen Überblick über die Zivilgesellzivilgesellschaftliche Funktionen erfullen. In For- schaft in Osterreich. Im ersten Abschnitt des Kapi-

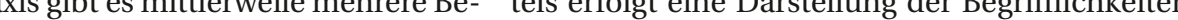
griffe, unter denen diese Organisationen zusammen- der insthutionellen Rahmenbedingungen und der gefasst werden.

\section{VERWENDETE BEGRIFFLICHKEITEN:}

Wer mit Organisationen der Zivilgesellschaft genau gemeint ist, hängt von der angewandten Definition
ab (Näheres dazu siehe Kapitel II). Weder die Beab (Näheres dazu siehe Kapitel II). Weder die Be-
griffe CSO (Civil Society Organisation) noch NPO griffe CSO (Civil Society Organisation) noch NPO
(Nonprofit Organisation) noch NGO (Nongovern(Nonprofit Organisation) noch NGO (Nongovern-
mental Organisation), die häufig weitgehend synomental Organisation), die hâufig weitgehend syno-
nym verwendet werden, stellen in Österreich eine konkrete Rechtsform dar - es handelt sich vielmehr um eine sehr heterogene Menge an Organisationen

mit unterschiedlichen Rechtsformen In der amtlChen Statistik gibt es erste Ansätze, NPOs als eigene bungen, die im Rahmen des CSI-RA durchgeführt quantitative Dimensionen der or Kanisiten Zive sellschaft auffezeigt werden, dieser Begriff verwen- Österreichs anhand verschiedener Dimensionen det wird. In Kapitel W kommt hingegen - in Anleh- beschrieben. Die weiteren Abschnitte befassen sich nung an CIVICUS - der BegriffCSO zur Anwendung mit den Themenbereichen Vereinigungsfreiheit, Versammlungsfreiheit Zugang zu finanziellen Ressourcen, Steuerrecht, Beschäftigung und Freiwilligenarbeit, Bereitstellung von Dienstleistungen und Transparenz bei der Vergabe von Leistungs- und Förderverträgen. Bei diesen Themen stehen vor allem die rechtlichen Rahmenbedingungen sowie deren Umsetzung in die Praxis im Vordergrund der Analyse. Der letzte Abschnitt von Kapitel III befasst sich mit der Wahrnehmbarkeit zivilgesellschaftlicher Wirkungen

Kapitel IV fasst die im Rahmen der Erhebungen diskutierten Stärken und Schwächen der österreichschen Zivilgesellschaft noch einmal zusammen.

Kapitel V enthält Handlungsempfehlungen, die in Kooperation mit der National Advisory Group (NAG) abgeleitet wurden.

GESCHLECHTERGERECHTE SCHREIBWEISE Im Sinne der geschlechtergerechten Schreibweise verwenden wir das Binnen-I (z. B. SpenderInnen), (z.B. Fördergeber) verzichten wir darauf im Sinne der Lesbarkeit.

\section{I.

\author{
CIVIL SOCIETY INDEX \\ PROJEKT \& \\ HERANGEHENSWEISE
}

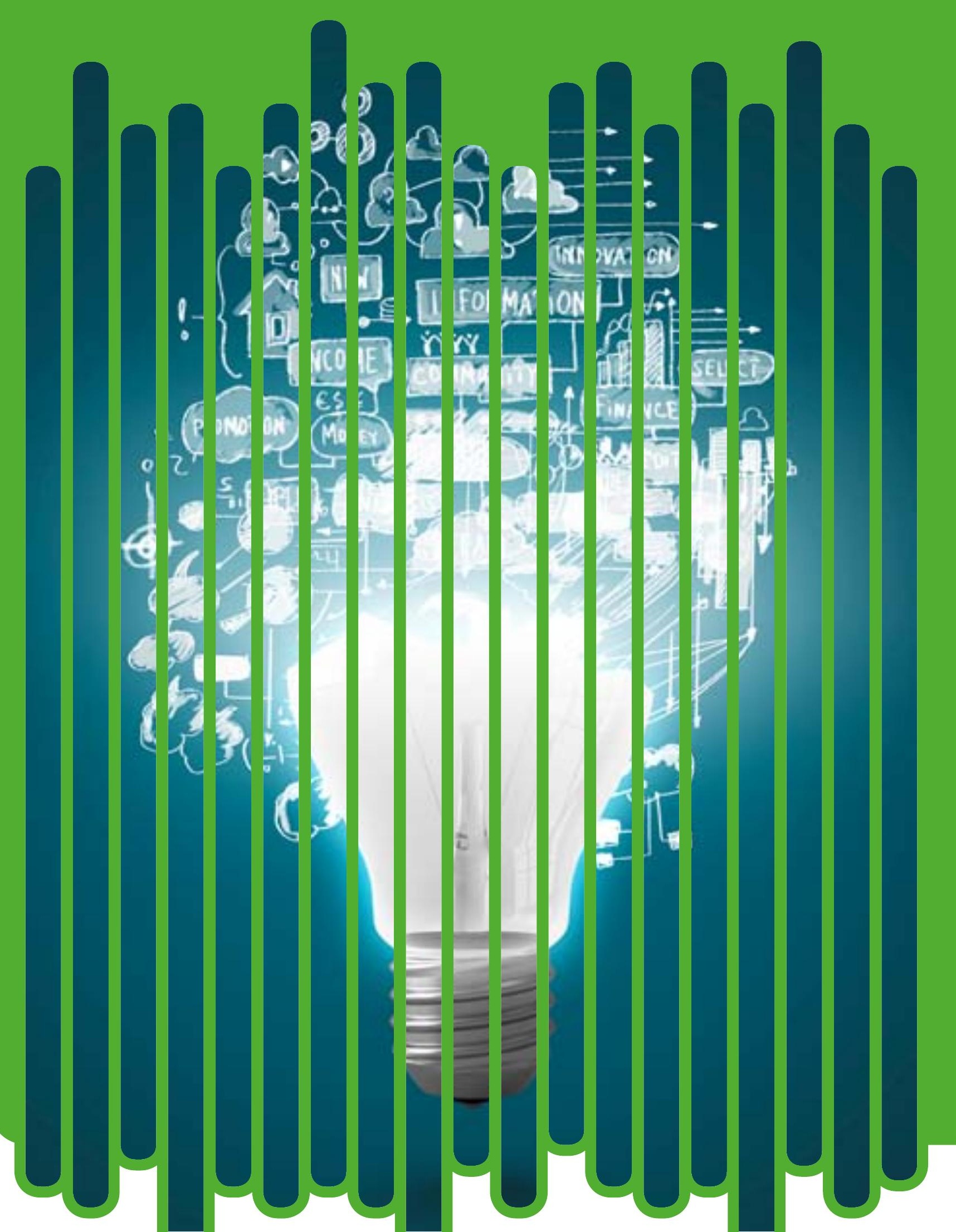




\section{CIVIL SOCIETY INDEX PROJEKT \& HERANGEHENSWEISE}

\section{Projekthintergrund}

7 ivilgesellschaftliche Organisationen tragen Un$\angle$ verzichtbares zu einer nachhaltigeren und solidarischeren Gesellschaft in Österreich und weltweit bei. Die Interessenvertretung Gemeinnütziger Organisationen (IGO) hat 2012 mit dem Bericht ,Zivilgesellschaft bewegt begonnen, das Wirken zivilgesellschaftlicher Initiativen und Organisatione systematisch zu untersuchen. Im Zuge der Arbeit
für den Bericht 2012 wurde deutlich, dass zivilgesellschaftliches Handeln und seine Rahmenbedi gungen in Österreich noch ungenügend beleuchtet

Es wurde daher entschieden, gemeinsam mit CIVICUS - World Alliance of Citizen Participation schaftsuniversität Wien die Rahmenbedingungen bzw. das politische und ökonomische Umfeld für z vilgesellschaftliches Engagement genauer untersuchen, konkret in Form des Civil Society Index - Rapid Assessment (CSI-RA).

\section{RAPID ASSESSMENT (CSI-RA)}

$\rightarrow \quad$ CIVICUS hat den CSI-RA aufbauend auf den jahrelangen Erfahrungen in 70 Ländern entwickelt. Der Forschungsprozess ist partizipativ, was zu einer Vergemeinschaftung von Erkenntnisinteresse, Forschungsprozess und Forschungsergebnis führt. Eine Besonderheit des CSI ist die am Ende des Projekts stehende Action Phase, die einen breiten Dialog des Berichts mit VertreterInnen von Politik, Medien und damit auch in der breiten Bevölkerung beinhaltet. Der CSI-RA ist ein flexibles Instrument, mit dem der 'Zustand` der Zivilgesellschaft in Österreich gemesse werden kann.

$\rightarrow$ Der CSI-RA ermöglicht eine Untersuchung der für die Zivilgesellschaft relevanten Faktoren, wie politische und ökonomische Rahmenbedingungen.

Der CSI-RA ist ent Instrument, das die Stärke, Kraft und Nachhaltigkeit von Zivilgesellschaft vergrößert, damit ihre Beiträge zu positivem sozialen Wandel intensiviert werde können.

$\rightarrow \quad$ Partizipation und Reflexion sind wichtige Elemente des CSI-RA Prozesses.

Im CSI-RA wird besonderes Augenmerk darauf gelegt, dass die Partner ihre Fähigkeit zur Zusammenarbei eigen, begreifen.

Im Zentrum der Untersuchung stehen im vorliegenden Bericht die Themen rechtliche Rahmenbedingungen/Rechtssicherheit und Wahrnehmbarkeit zivilgesellschaftlicher Wirkungen.

\section{Herangehensweise und}

\section{CSI Implementation}

er vorliegende CSI-Ansatz verbindet wissenschaftliche Erhebung mit Reflexion und ge-

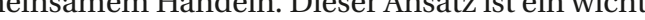
ger Referenzrahmen fúr die gesamte Arbeit. Als solches geht es im vorliegenden Bericht nicht da, Wissen um seiner selbst Willen zu produzieren sersersungen fur kens zu erzielen.

Am I4. Oktober 2013 fand an der Wirtschaftsuniversität Wien ein eintägiger Workshop statt, an dem neben Mitgliedern der Advisory Group außerdem noch Ulla Ebner (Journalistin beim Radiosen-

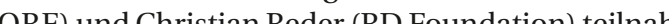

Der Workshop (Adaptation Meeting) diente der Identifikation möglicher Stoßrichtungen für die Forschung. Es wurden die Themen. , okonomische lung der Erfolge der zivilgesellschaftlichen Organsatione Forschungsgebiete identifiziert

In den Wochen nach dem Adaptation Meeting wurden sechs relevante Themenfelder entschieden Demokratie, BürgerInnen und Menschenrechte, Steuerrecht, Beschäftigung/Sozialversicherung, Vergaberecht und Förderungen sowie Wahrnehmbarkeit zivilgesellschaftlichen Wirkens. Der Schwerpunkt der Erhebung liegt auf den rechtlichen Rahmenbedingungen und der damit verbundenen Praxis. Den Themen sind Subthemen untergeordnet, die jeweils ein Unterkapitel im nachfolgenden Kapitel III bilden (siehe auch Abbildung I).

Als Grundlage für eine Einschätzung der Rahmenbedingungen wurden Indikatoren überlegt und recherchiert, mithilfe derer die Themenfelder systematisch untersucht werden konnten. Die vom European Center for Non-Profit Law (ECNL) für die Monitoring Matrix on Enabling Environment ${ }^{\mathrm{e}}$ erarbeitete Liste an Indikatoren zur Erfassung rechtliÖsterreich außerordentlich geeignet und als für Osterreich außeronentich gesignet und wurde un sowie Indikatoren von ECNL und Indikatoren wurde ein erstes Set fixiert. Errebnis waren sechs Themenfelder mit neun Subthemen einem nächsten Schritt wurde dieses Indikan. In Set von Mitgliedern der National Advisory Group ergänzt und abgestimmt. Die Indikatoren sind im jeweiligen Kapitel dargestellt und differenzieren zwischen rechtlichen Bestimmungen und der Umsetzung in die Praxis.

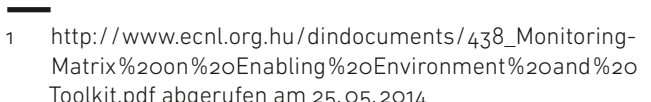

Abbildung 1:

SCHWERPUNIKTTHEMEN DER STUDIE

\begin{tabular}{|c|c|c|}
\hline Demokratie & 1. Demokratie & Desk-Research \\
\hline Bürgerlnnen- und & 2. Vereinigungsfreiheit & Desk-Research, 6 Interviews \\
\hline Menschenrechte & 3. Versammlungsfreiheit & Desk-Research, 6 Interviews \\
\hline & 4. Zugang zu finanziellen Ressourcen & Desk-Research, 2 Interviews \\
\hline$\overline{\text { Steuerrecht }}$ & 5. Steuerrecht & Desk-Research, 7 Interviews \\
\hline $\begin{array}{l}\text { Beschäftigung/ } \\
\text { Sozialversicherung }\end{array}$ & 6. Beschäftigung und Freiwilligenarbeit & Desk-Research, 7 Interviews \\
\hline Vergaberecht und & 7. Bereitstellung von Dienstleistungen & Desk-Research, 8 Interviews \\
\hline Förderungen & $\begin{array}{l}\text { 8. Transparenz bei der Vergabe von } \\
\text { Leistungsverträgen und Förderungen }\end{array}$ & Desk-Research, 8 Interviews \\
\hline Wahrnehmbarkeit & $\begin{array}{l}\text { 9. Wahrnehmbarkeit zivilgesellschaftlicher } \\
\text { Wirkungen }\end{array}$ & Quantitative Erhebung \\
\hline
\end{tabular}

Aufgrund des für die Beantwortung der Fragenotwendigen ExpertInnenwissens und der in vielen Fällen gegebenen Objektivierbarkeit durch Rechtsquellen wurde als methodische Hersearch undinsgesamt rund 20 Interviews mit RechtsexpertInnen und VertreterInnen von CSOs zu fokussieren. Die Indikatoren bildeten die Grundlage für die Auswahl von InterviewpartnerInnen und die Erstellung der jeweiligen Interview-Leitfäden. Aufgrund der Heterogenität der Themenbereiche mussten diese zwischen verschiedenen InterviewpartnerInnen aufgeteilt werden, wobei manche InterviewpartnerInnen (insbesondere die CSO-VertreterInnen) mehrere Themenfelder abdecken konnten.

Im Zeitraum Februar-April 2014 wurden 24 Interviews mit II Rechtsexperthnnen und I3 VertreterInnen von CSOs geführt. Erste Zwischenergebnisse der Erhebungen wurden im Rahmen eines Treffens der NAG Ende März diskutiert. Ergänzend wurde Feedback von Mitgliedern der NAG, die nicht an dem Treffen teilnehmen konnten schriftlich und in persönlichen Treffen eingeholt. 


\section{Grenzen der Studie}

ie Studie basiert einerseits auf einer Zusammendaten song von Sekundärliteratur und Sekund Die ausgewählten Indikatoren dienten als Orientierung in der Recherche sowie bei der Erstellung der Interviewleitfäden. Die gewählten Themenbereiche sind sehr heterogen und die Indikatorenliste sehr umfassend, weshalb nicht alle Indikatoren gleichermaßsen behandelt, sondern in den Interviews verschiedene Schwerpunkte gesetzt wurden. Zudem sind die Bedingungen je nach Tatigkeitsfeld der betreffenden CSOs oft sehr unterschiedlich - dies trifft insbesondere auf die Ausschreibungs- und Förderbedingungen $\mathrm{zu}$, so dass die gemachten Aussagen nicht fur alle CSOs generalisiert werden konnen. Obwohl wir auch Interviews mit Vertreterlnnen des Sport- sowie des Kunst-und Kulturbereichs geführt haben, lag hier ein deutlicher Schwerpunkt bei Vertreterlnnen des Sozial- und Gesundheitsbereichs. bes bedeutet, dass die Ehschatzung der RahmenDen Singen reprentasiven Erhebung quantitatizeigte unters billicher Ausprïgung fïr vile de befragten CSOs zutreffen und a NAG bestätigt wurden.

\section{II. ZIVILGESELLSCHAFT IN ÖSTERREICH}

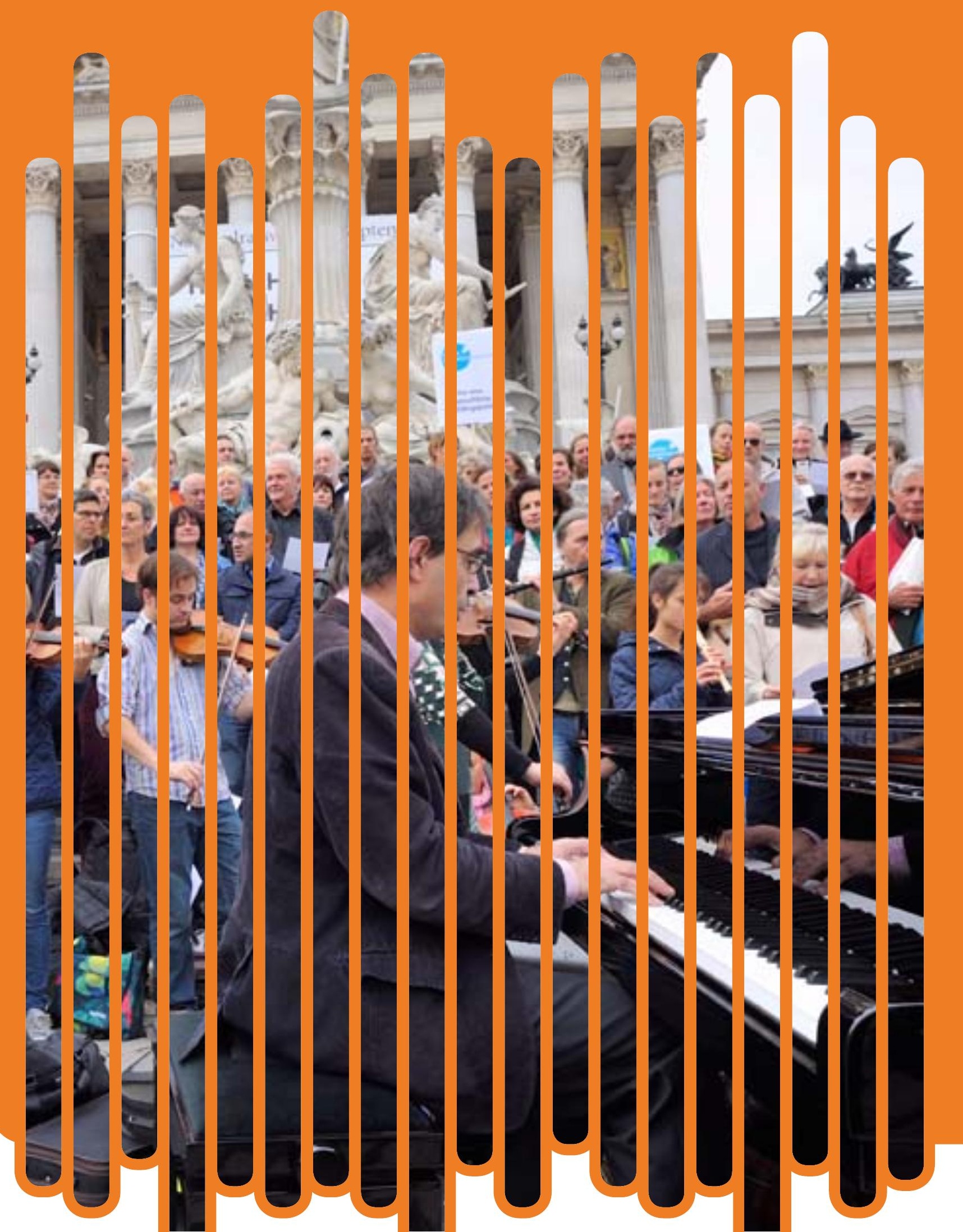




\section{ZIVILGESELLSCHAFT IN ÖSTERREICH}

1. Das Konzept der Zivilgesellschaft $Z_{\text {Wirtschaft und Privatem, in der Menschen ihre }}^{\text {ivilgesellschaft meint eine Späre }}$ Anliegen selbst vertreten und zu gestalten versuchen, meist verbunden mit Ideen von Partizipation, Priller 2007). Häufig wird die Zivilgesellschaft in Zusammenhang mit NPOs (Nonprofit Organisations) bzw. NGOs (Nongovernmental Organisations) gebracht (und zunehmend als CSOs (Civil Society Organisations) bezeichnet), also mit privaten, nichgewinnorientierten, formalen Organisationen $\mathrm{m}$ einem Mindestmaß an Freiwilligkeit. Kontrovers is wie weit Anforderungen an Pluralität, Toleranz und
Diskursivität konstitutives Element sind. Auch natiDiskursivität konstitutives Element sind. Auch nati-
onalistische und fundamentalistische oder sogar rechtsradikale AkteurInnen agieren in dieser - dann ls bivil society bezeichneten (Chambers/ Kopstein 200I)

Die Trennlinie zwischen Zivilgesellschaft und NPO-Sektor ist oft diffus. Auch die Soziologie hat eine unscharfe Begrifflichkeit (Klein/Rohde 2003) Sektor werden oft synonym verwendet (Sims Sektor werden oft synonym verwendet (Sims
2013a) und Forschung unter dem Titel Zivilgesellschaft wird oft auf die Erfassung von NPOs eingeschränkt. Auch wenn v. a. freiwillige Vereine oft Recht als organisatorischer Kern oder Infrastruktu der Zivilgesellschaft charakterisiert werden (Adlo 2005; Birkhölzer et al. 2005), und es historisch ein starke Uberschneidung des NPO-Sektors mit Ne werken sozialer Bewegungen gibt, kann diese Vo gangsweise der Vielfalt

Orientiert an der auch im internationalen $\mathrm{Ce}$ brauch gängigsten Definition (Salamon/Anhei 1992) werden NPOs hier definiert als Organisationen, die

durch ein Mindestmaß an formale Organisation gekennzeichnet sind

private, nicht staatliche Organisationen sind;

keine Gewinne an Eigentümer oder Mitglieder ausschütten;

en Minimum an Selbstverwaltung bzw.

Entshein

gekennzeichnet sind.

Hier soll Zivilgesellschaft definiert werden als die Summe von AkteurInnen und Handlungen, die ein Mindestmaß an Autonomie von Markt und Staa aufweisen, die auf die Gestaltung politischer Prozesse und/oder sozialer Lebensbedingungen richtet sind und im Rahmen kollektiven Handelns stattfinden. In anderen Worten: , Civil society is an open arena of participation, located beyond the fuzzy boundaries of state and market in which diferent types of individuals, groups, and organiztions cooperate or compete for visibility an relevance, in the pursuit of collective (though no necessarily shared) political and social goals and animated by a variety of values and interests (Fior monti/Thümler 20I3). Soziale Bewegungen, die an Themen kondensieren und sozialen Wandel durch Protest herbeifuhren wollen, konnen als Sonde form zivilgesellschaflichen Engagenents charakterisiert werden, die sich durch besonders hohen Grad an Vernetzung, Aktivitäts- und Interaktionsa

\section{ZIVILGESELLSCHAFT IN ÖSTERREICH -}

\section{促}

eiten der österreichischen Zivilg den nor österreichischen Zivilgesellschaft we allgemeiner Hintergrund gegeben werden.

In Bezug auf empirische Forschung wird in Österreich unter Zivilgesellschaft schwerpunktmäßig der NPO-Sektor behandelt, daher gibt es v. a. Datenmaterial dazu. NPOs stellen alledings Civil Society Organisations einen wesentlichen Teil der österreichischen Zivilgesellschaft dar. Soziale Bewegungen haben angefangen von der ArbeiterInnen- und der bürgerlichen Frauenbewegung bis zu neuen Bewegungen, wie der Umweltbewegun lange Tradition, die sich aber auch rasch in der Etablierung spezifischer NPOs ausdrückte.

NPOs spielen in Österreich eine große Rolle für das Alltagsleben vieler Menschen, für die politische Kultur und auch für die ökonomische Entwicklung. Beiträge für das Alltagsleben vieler Mensche werden oft nicht wahrgenommen. Aus den vielfältigen Leistungen seien daher hier ein paar ausgewählte Zahlen genannt. So wurden 2010 insgesam über I5,5 Millionen Leistungsstunden in mobilen Diensten geleistet und dabei insgesamt I27.89 Menschen betreut, in 73 Beschäftigungsprojekten der Caritas wurden 894 Arbeitsplätze für Langzeiarbeitslose geschaffen, die SOS Kinderdörfer haben 20 I I an die 6.400 Personen in Österreich betreu weltweit ein dauerhaftes Zuhause für ca. 80.00 Kinder und Jugendliche geschaffen und insgesam jährlich mehr als zwei Millionen Menschen mi inren Programmen erreicht. Licht fur die Welt hat geren und die Einsatzfahrzeuge des Arbeitergameriter meter pro Jah zune mehr als 18 Mithonen Kiloum die um die Erde entspricht, durchsc mal pro Tag angefordert.

Der Sektor hat also eine Fülle an sozialen Wi gesellschaftliche Vielfalt, Partizipation und Innovation. Die meisten dieser Wirkungen sind nich direkt messbar-sie discheinen in kein sind nich Statistik. Insbesondere aber die hohe ökonomische Bedeutung des Sozialbereichs wird systematisch unterschätzt - ManagerInnen aus Sozialorganisationen beklagen immer wieder, dass ihre Branche als 'Fass ohne Boden` bzw. als rreiner Kostenfaktor wahrgenommen wird.

\section{RECHTLICHER RAHMEN}

erglichen mit anderen Ländern sind rechtliche Rahmenbedingungen tendenziell freundlich ‘ür NPOs, BürgerInnenrechte und Demokratie sind relativ gut ausgeprägt. Spezielle Restriktionen und Verbesserungsmöglichkeiten gibt es natür

Es gibt im österreichischen Rechtssystem keine eigene Kategorie für NPOs. Der NPO-Status ist daher keine spezifische Rechtsform. Eine Reihe an Steuergesetzen ist allerdings dafür relevant, insbesondere Regeln zur Gemeinnützigkeit. Für NPOs kommen folgende Rechtsformen in Frage: Verein,

Stiftung, gemeinnützige
sellschaft und GmbH.

Eine Non Profit Organisation kann theoretisch jede Rechtsform annehmen, ausgenommen eine die nach Profitorientierung verlangt. (z. B. offene Handelsgesellschaft [OHG] oder Kommanditgesellschaft [KG]) Trotzdem sind die meisten NPOs, Kultur- und Sportorganisationen, Interessenverbände, politische Parteien und Selbsthilfegruppen einge schos (siehe unau zur) (siche sie keine strengen Anforderungen was Jahresberichte oder Rechnungslegung betrifft. Tatsächlich ist die Vereinsbewegung eine wichtige Basis für den österreichischen Non Profit Sektor. I867 wurde das verfassungsmäßige Recht sich in Vereinen zu das nisieren gewährt und seitdem weitreichend genutzt Aktuell gibt es mehr als I 10.000 Vereine in Österreich, davon gelten fast alle als Non Profit Organisationen. Im Juli 2002 wurde das Vereinsrecht das erste Mal seit 1952 novelliert, was zu strengeren Regelungen für große Vereine führte, z. B. strengere Regeln in Bezug auf Rechnungslegung und erweiterte Haftung für Vereinsmitglieder.

Neben der Vereinsform werden NPOs auch häufig als Stiftungen eingetragen, z. B. operative oder fördernde Stiftungen in den Bereichen soziale Dienstleistungen, Gesundheit, Bildung und Forschung. Trotzdem ist die Rechtsform der Stiftung in Osterreich nicht sehr gangig, was sich teilweise dadurch erklären lässt, dass private Stiftungen erst seit 1993, also seit der Einführung des Privatstiftungsge setzes, zugelassen sind. NPOs können auch die Rechtsform einer Genossenschaft annehmen. Jedoch hat sich die Geneser Genossenschaften mit der Zeit stank verandent, da viele nitllerwelle profiProfich des genoshenschaftichen Sektors (Wohnbau Genossenschaften) kann immer noch als nicht profiorientiert bezeichnet werden. Andere Rechtoformen wie Gesellschaft mit beschränkter Haftung (GmbH) oder Aktiengesellschaft (AG) werden selten von Non Profit Organisationen genutzt.

\section{ESTIMMENDE GESELLSCHAFTLICHE}

ektor ist nicht nur relativ roß, sondern auch sehr heterogen. NPOs untercheiden sich nach Größe, Rechtsform, Tätigkeitseld, finanzieller Situation, Struktur und andere Merkmalen. Die folgenden Prinzipien und Besonderheiten sind wesentlich für die konkrete Gestalt des Sektors:

Föderalismus und Selbstverwaltung

ie föderalistische Struktur Osterreichs, d. h. die Giederung nach neun Bundeslândern, spiegelt sich auch im Aufbau des NPO-Sektors wider. Viele NPO gieren auf Landesebene, haben ihre Ansprechparter in der Landesverwaltung und agieren auf Basis endesspezifischer Sozialgesetze und Finanzieungsmodi. Bundesweite NPOs haben gegenüber hren Landesorganisationen oft eine relativ schwacio Stellung. Auf Landesebene agierende NPOs konnen sich von ihren Schwesterorganisationen in

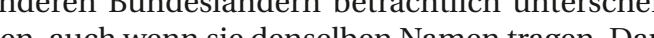
cin ing eine withe Basis des Nonprofit Sektors in dic sich Wuns nach Autono (i) rwaltung ausdrückt (Pennerstorfer et al. 20I3).

\section{Korporatismus}

. ohen Einfluss und z. T. den Charakter von gesetzchen Interessenvertretungen (z. B. Wirtschaftsandwirtschaftskammern), bei denen die Mitgliedschaft gesetzlich verpflichtend ist. Teilweise handelt sich um freie Berufsvereinigungen wie den Osterung der Osterreichischen Industrie. Die Beteiliang dieser Interessenvertretungen an politischen ntscheidungsprozessen wird als Korporatismu

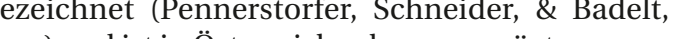
Un in Ö. NPOs úben aufgrund des Korporatisme in Ostereich einen im internationalen Vergleich stark ausgeprägten politischen Einfluss aus. Wir die auß ungen agierenden NPOs nicht leicht, ihre gesellchaftliche Position zu definieren und Einfluss zu enver ld anderer NPOs hineinwirken slbst Leistungen anbieten oder unterstïtzen (z. B. Kulturförderung der Arbeiterkammern), sei (z. B. deren standespolitisches Lobbying die wirtschaftlichen Grundlagen mancher NPOs wesentlich betrifft. Drittens sind auch im Nonprofit Sektor sowohl die Seite der ArbeitgeberInnen als auch die der 
ArbeitnehmerInnen zunehmend in Interessenvertretungen organisiert.

Zudem sind v. a. große oder traditionsreiche NPOs oft in Dachverbänden zusammengefasst, die sowohl innerhalb einer Organisation (etwa als Bunbände) als auch ür bestimmte Branchen organistionsübergreifend (z. B. Bundessportorganisation Landesfeuerwehrverbände, Dachverbände von Sozial- und Gesundheitsdiensten) gebildet werden. jüngster Zeit haben sich darüber hinaus auch branchenübergreifende Dachverbände entwickelt (z. B. die Interessenvertretung Gemeinnütziger Organisaan bestimmten Aufgaben orientiert (z. B. Fundrasing, ArbeitgeberInnenfunktionen etc.).

Schließlich stellen die starken Berufsverbände für NPOs, die sich selbst primär als Interessenvereine Art unausgesprochen Referenzgröße dar.

\section{c.Weitgehende Nähe zu politische Parteien}

Der österreichische Parlamentarismus ist durch ein System der reprasentiven Parteiendentratie gereichen politischen Parteien (SozildemoktischPartei [sPö], ÖsterreichischeVolkspartei [ÖvP]) pagen das gesellschaftliche System.

Ein großer Teil der traditionsreichen und wirtschaftlich starken NPOs steht direkt oder indirek im Einflussbereich der Großparteien. In manchen Leistungsfeldern des NPO-Sektors gibt es sog eine politische, Marktaufteilung $<$ zwischen den Einflusssphären neben denen unabhängige Organistionen oft nur schwer überleben können. So komm es, dass noch heute nicht nur im Sozialwesen, sondern auch im Sport, bei den Alpinvereinen, im Katastrophenhilfsdienst, bei den Interessenvertretungen der AutofahrerInnen, bei Kulturvereinigungen und Erwachsenenbildungseinrichtungen sowie in Genossenschaftswesen schwarzer (ÖVP) und 'roter (SPÖ) NPOs existieren. Tendenziell lost sich diese zum Teil zu beobachtende Dichotomie des NPOSektors auf. Bestehende NPOs sind vermehrt au politische Unabhängigkeit bedacht, ebenso entstehen neue, politisch unabhängige NPOs.

Generell gibt es wichtige Wechselwirkungen zur Politik. Einerseits üben manche NPOs direkten Einfluss auf Gesetzgebung und Verwaltung aus (z. B. bei der Definition von Forderngskriterien). Andepolitischerich politsch tiert auch eine viefütige und üb Szen a und lokalenVereinen, die sich so lenge von den oben skizzierten Machtstrukturen abkoppeln können, sie keinerlei umfassenden Ressourcenansprüche entwickeln und sich auf sachlich und/oder region klar definierte Aufgaben beschränken. Dieser Teil des Sektors kann zum Teil auf langü̈hrige Traditionen zurückblicken (z. B. in Form der lokal agieren- den Kulturvereinigungen wie Musikkapellen, Sportvereine etc. zialen Bewegungen wie BürgerInneninitiativen Selbsthilfegruppen etc. zum Ausdruck.

Die Rolle der Kirche(n)

eben den politischen Parteien prägen auch die Kirchen - vor allem die katholische - wichtige Teile des Nonprofit Sektors. Zunächst sind kirchliche Institutonen mit den von ihnen angebotenen oder organtien tungensers als Bestandteile des Nonprofit Sektors erbände, z. B. im Familien- oder Sozialwesen.

Neben den religiơsen Dienstleistungen treten Kirchen vor allem im Sozial-, Gesundheits- und Bildungswesen als Anbieter von Dienstleistungen auf, wo sie in manchen Arbeitsfeldern die größten Anbieter innerhalb des Nonprofit Sektors sind. Wichtige Beispiele darur sind das Erziehungswesen (Vorschulerziehung, Schulwesen, außerschulische Jugencitic sowie sozialen

Bei den Sozialen Diensten agieren die Kirchen Ber Organisation (z B. Caritas, Diakonie) Dazkon作 tionen (z. B. PfadfinderInnen, Malteser Hilfsdienst, Kolpingwerk). Manche NPOs stehen sowohl im Vorfeld politischer Parteien - insbesondere der övp als auch der Kirchen (z. B. katholische Studentenverbindungen) (Pennerstorfer et al., 2013).

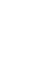

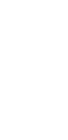

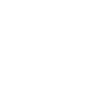

.

.

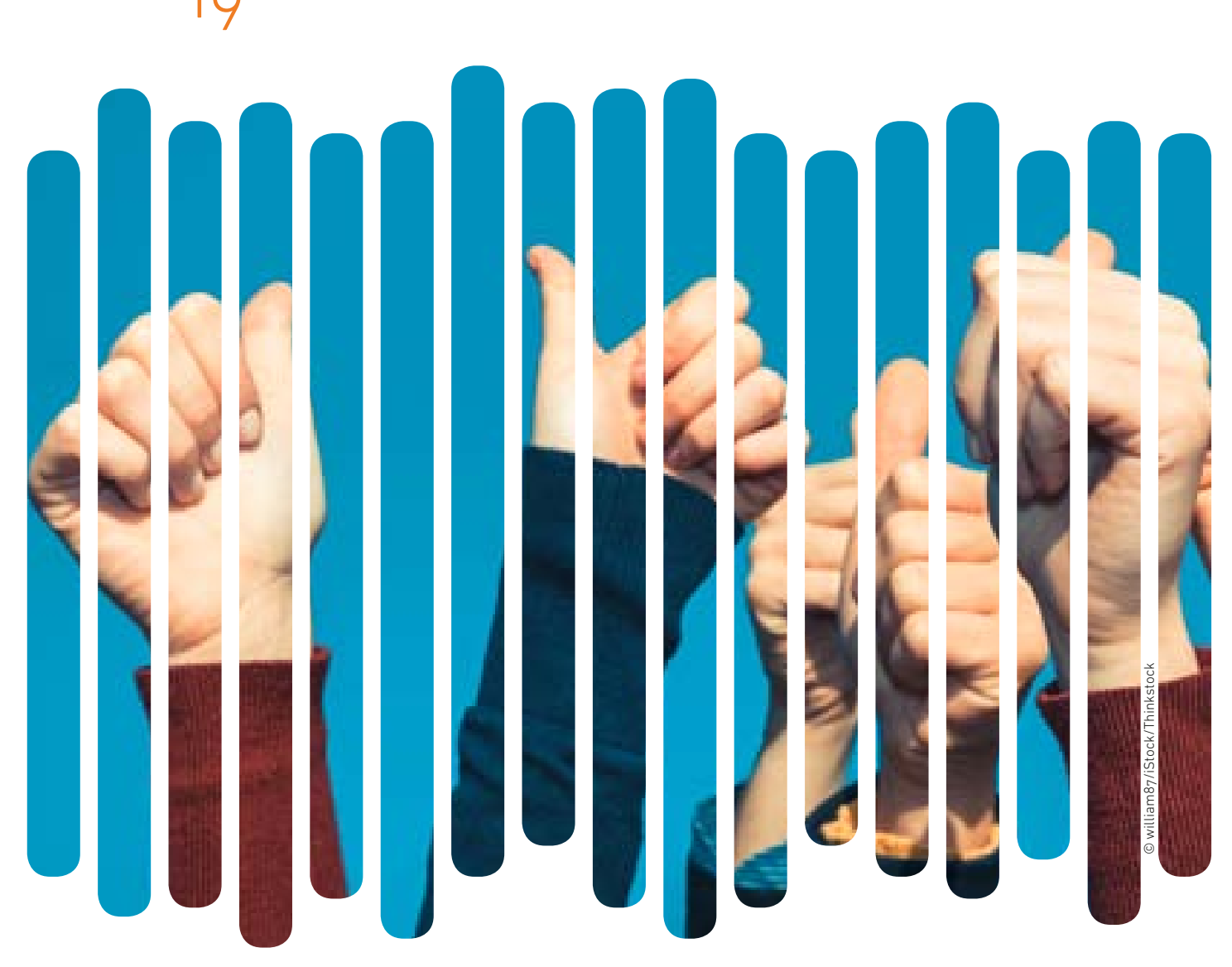

e. Genossenschaften und Gemeinwirtschaft

Genossenschaften spielten in der Entwicklung des Sektors eine große Rolle, haben in den letzten Jahren aber zusehends an Bedeutung verloren. Auch in Österreich hat sich das Genossenschaftswesen im heutigen Sinn in der Mitte des 19. Jahrhunderts als eine der Antworten auf die massiven wirtschaftlichen und sozialen Umbrüche dieser Zeit entwickelt. Der genossenschaftliche Grundgedanke - der freiwillige Zusammenschluss zur wirtschaftlichen Selbsthilfe hat seit dem ersten osterreichischen Genossen-
schaftsgesetz (1873) in vier großen Aktionsbereischaftsgesetz (1873) in vier großen Aktionsbereichen - namlich Landwirtschaft, Gewerbe, Konsum Spitzenverbänden des Genossenschaftswesens zum Ausdruck kommt.

Während die traditionsreichen Genossenschaften durch Kommerzialisierung, Konzentration und rescistion tätswandele nicht mehr als Teil der Zivilgesellschaft angesehen werden können, hat der Genossenschaftsgedanke im Klinen in den letzten Jahren werte Ren in den letron Jün wert ist dabei fließend (Pennerstorfer et al, 2013).

\section{f. Vereinswesen}

In der österreichischen Gesellschaft spielt das freie Vereinswesen eine tragende Rolle. Historisch gesehen waren die Möglichkeiten, Vereine zu bilden über weite Strecken ein Ausdruck der Liberalität des politischen Systems. Erst mit dem Staatsgrundgesetz von I867 wurde die Vereins- und Versammlungsfreiheit in den verfassungsrechtlichen Grundrechtskatalog aufgenommen. Das Vereinswesen ist insbesondere für die Alltagskultur in Osterreich von großer Bedeutung. Vereine können relativ leicht und ohne großen Kapitaleinsatz gegründet werden (siehe auch Kapitel III/2).

g. Stiftungen

Osterreich nimmt hinsichtlich der Stiftungen eine Sonderstellung ein, da es hier nicht nur gemeinnützige sondern auch eigennützige Stiftungen gibt. Seit 1993 gibt es die Möglichkeit, Privatstiftungen zu
gründen, die nicht zwingend gemeinnützig sein müssen. Insgesamt sind etwa nur $20 \%$ aller in Österreich existierenden Stiftungen 'vorrangig، gemeinnützig (vgl. [H. Schneider et al., 20I0]: I I). Die gemeinnützigen Stiftungen fördern v. a. Aktivitäten im Bereich Bildung, Soziales und Kultur. Die im Dezember 2013 angelobte Koalitionsregierung aus SPO und OvP hat in ihr Arbeitsprogramm das Vorhaben aufgenommen, eine neue rechtliche Grundlage für gemeinnutzzige Stiftungen zu schaffen und so zubatzlche Geldmittel fưr bestimmte Bereiche zu mobuch bei anderen NPOs von den Finanzbehörde zu bei aren NPOs von den Finanzbehơrden keinem öffentlich zugänglichen Register erfasst.

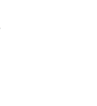

.

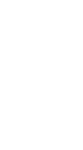




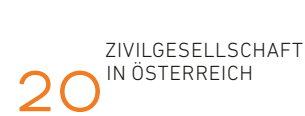

2. Historische Entwicklungen

er Non Profit Sektor in Österreich hat eine lange ren Ursprung im I9. Jahrhundert. Er hat daben ihmeinsame Wurzeln und Traditionen mit Non Profit Sektoren in anderen zentraleuropäischen Staaten,
die Teil der österreich-ungarischen Monarchie waren, wie Ungarn, der Slowakei und Tschechien. Eine Reihe von Faktoren haben die Entwicklung und Charakteristika des Sektors in Österreich geprägt. Vor den zwei Weltkriegen war das österreichidie Aristokratie, das Militär und die Kirche einen enormen Einfluss auf die Gesellschaft hatten. Da diese Gruppen in enger Verbindung zum Staat standen, führten ihre gemeinsamen Interessen zu einem starken Etatismus und einer dominanten Stellung für Beamte in Osterreich. Dieser Hintergrund be einflusste die Größe, Form und Charakteristika des Sektors und resultierte in engen Beziehung
viele NPOs bis heute mit dem Staat haben.

Gleichzeitig wurden viele zivilgesellschaftliche Organisationen in Verbindung zur Arbeiterbewegung gegründet. Folglich waren einige Vorläufer der heutigen großen und etablierten NPOs, z. B. Gewerkschaften oder große im sozialen Dienstleisungsbereich tätige Organisationen, ursprünglich der Kirche. Viele Vereine wurden 20. Jahrhunderts von der sozialdemokratischen Bewegung den konservativen Christdemokraten oder der Kirche gegründet. Damit versuchte man die Gefolgsleute auf verschiedenen Ebenen an die jeweilige Ideologie zu binden.

Viele Selbsthilfegruppen und private Wohlfahrtsverbände können als Vorläufer der heutigen NPOs bezeichnet werden. Für die ideologischen Gruppierungen war entscheidend, dass ihre Mitglieder an ihrer jeweiligen Partei oder Institution festhielten - Mitglieder wurden 'von der Wiege bis zur Bahre` protegiert und betreut. Zwischen den politischen Lagern kam es kaum zu Austausch: Mitgliedern der Sozialdemokraten war es sogar verboten Sportvereinen beizutreten, die mit den konservativen Christdemokraten oder der Kirche in Verbindung standen.

In den I930er Jahren war die Zivilgesellschaft in zwei Teile gespalten. Viele NPOs wurde vom Nazi Regime verboten und horten damit entweder auf zu existieren oder mussten im Untergrund weiterarvom Rerime vereinn wm, um politische Aufgaben zu erfüln Die Mitlied, un in dien zum wesentich a da bei die Nazi-Kulturund tik zu verbreiten.
Nach 1945 wurden die alten Traditionen des ektors wieder aufgenommen, wenn auch leich schaft spielte fortan eine wichtige Rolle in der östereichischen Gesellschaft. Weiters trug die Soziaartnerschaft, die von RepräsentantInnen derbeide politischen Lager aufgebaut wurde und somit die neokorporatistischen politischen Inhalte institutionalisierte, wesentlich dazu bei, die Kluft zwische dem linken und dem rechten Flügels des NPO-Sekors zu überbrücken. Die zwei ideologischen Bewegungen waren nicht mehr in dem Ausmaß vonein政 er Fall gewesen war. Nichtsdestotrotzwar ihre Mach norm und viele Vereine wurden als Vorfeldorgan sationen für eine der politischen Parteien oder die Irrche genutzt. Das fuhrte zu der weitverbreitete Annahme, dass in Osterreich nichts ohne die Untestützung einer der beiden Großparteien erreich gung ausgenommen, waren Non Profit Institutiogung ausgenommen, waren Non Profit Institutiototrotz haben sie ihr Potenzial sozialen Wandel herbeizuführen, behalten

Erst viel später, in den I980er Jahren und mit em Erwachen neuer sozialer Bewegungen, entNPOs, die weder mit den etablierte varen Diese Organisach mit der Kirche verbunden Bereichen: Frauenbewegung, Umwelt oder Menchenrechte Entwicklungszusammenarbeit Mnd inernationale Beziehungen aktiv. Sie wurden für die osterreichische Zivilgesellschaft sehr wichtig und haben deren Kultur und Gewohnheiten geprägt. We gen ihrer Distanz zum Establishment. erhielten sie jedoch weniger öffentliche Förderungen. Dahe wird ihre Bedeutung in Statistiken über den NPOSektor nicht widergespiegelt. Dieses Segment der sterreichischen Zivilgesellschaft ist noch immer sehr instabil.

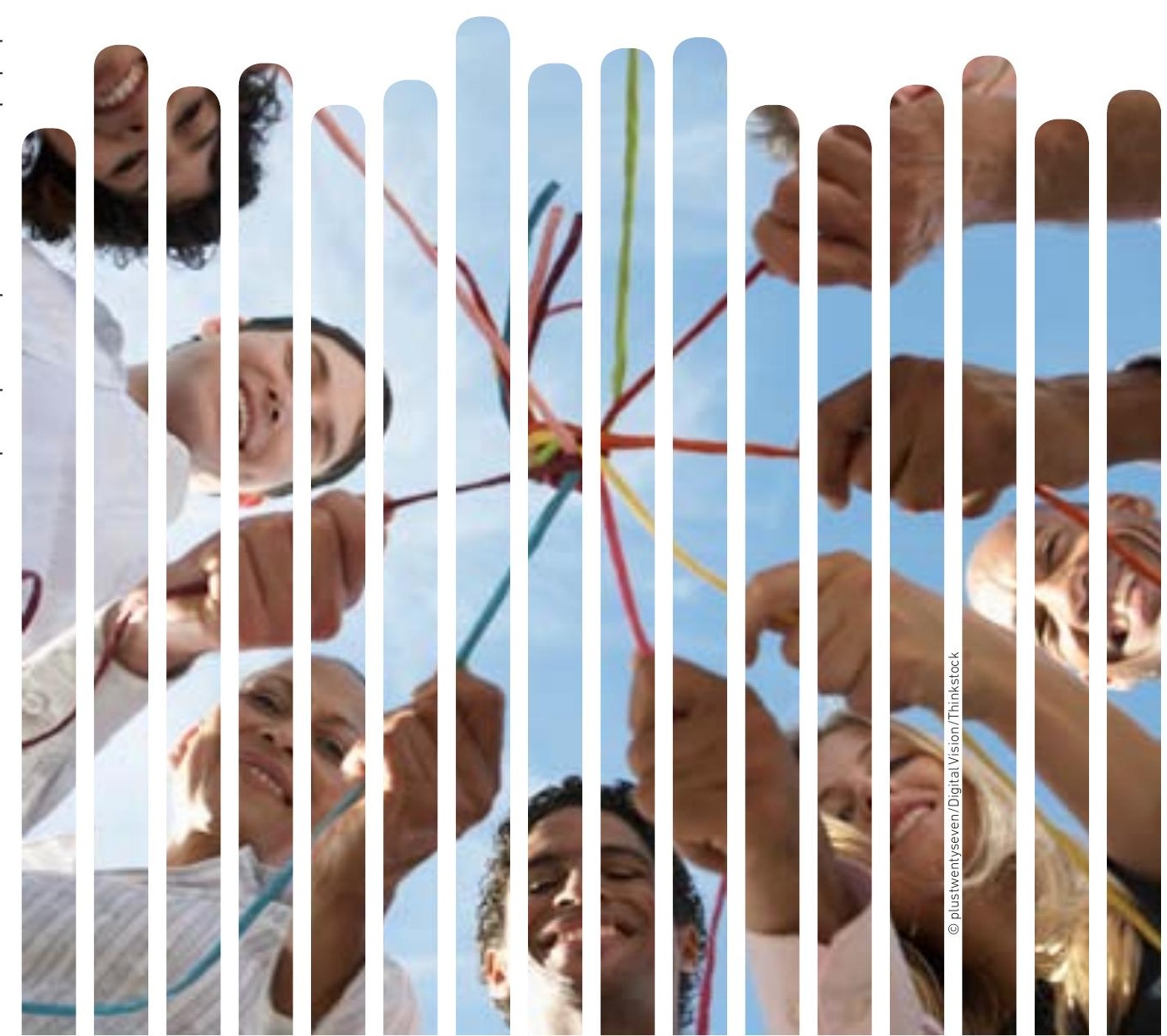

3. Abbildung der Zivilgesellschaft Der NPO-Sektor in Österreich

Verlässliches Datenmaterial gibt es nur für den NPO-Sektor. Für nichtformalisiertes zivilgesellmonstranten, AktivistInnen und BloggerInnen, die am politischen Diskurs und an Entscheidungsprozessen teilhaben wollen, sich aber dem Zugriff vo etablierten Bewegungen und Institutionen wie Pa teien, Gewerkschaften, aber auch NPOs, entziehen (Anheier 2013; Simsa 2013b) gibt es keine Statistiken.

Der folgende Abschnit konzentriert sich al auf Nonprofit-Organisationen. Vorausgeschick werden muss, dass auch die Datenlage in Bezug au NPOs unbefriedigend ist (Schneider/Haider 2009). Im Folgenden werden die derzeit aktuellsten verfügbaren Daten aus unterschiedilchen Studien un Statistiken angeführt, z. T. kann dabei nur auf die Sozialwirtschaft Bezug genommen werden, die gemessen an Beschättigten einen großen Anteil an NPO-Sektor einnimmt, oder auf jene NPOs, di Dienststaten sind, also zumindest eine Person baten in diesem Kapitel anders genannt, sind 2013 entnommen.

\section{ANZAHL DER ORGANISATIONEN}

Vereine sind quantitativ betrachtet die mit Abstand wichtigste Rechtsform österreichischer NPOs. Im Jahr 20I0 waren in Österreich II6.556 Vereine in Osterreich registriert (Statistik Austria 2012). Die fast verdreifacht. Im Jahr I960 waren 42.269 Vereine registriert, seitdem gibt es eine kontinuierliche Zunahme von Vereinen in Österreich.

Im Jahr 2003 waren die größten Gruppen an ereinen Sportvereine, Sparvereine und Kulturvereine (Musik, Theater, Gesangsvereine) - seit dem neuen Vereinsregister werden die

Insgesamt gab es 2008 3.I4I Privatstiftungen, von denen lediglich 210 rein gemeinnützig sind sowie 34 Sparkassenstiftungen mit vorrangig gemeinnützigen Zielsetzungen. Zusătzlich gab es 246 Landes und 223 Bundesstiftungen, die ausschließlic gemeinnützige oder mildtätige Zwecke verfolgen
dürfen. Demnach sind nur ca. 20\% aller Stiftungen dürfen. Demnach sind nur ca. $\mathbf{2 0} \%$ aller Stiftungen
vorranging der Gemeinnützigkeit verschrieben vorranging der Gemeinn
(Schneider et al. 2010).

Die Anzahl der NPOs mit anderen Rechtsformen, alsogemeinnützige Aktiengesellschaften, $\mathrm{C}$ angegeben werden, da nur jene erfasst werden wo der Begriff gemeinnützig im Namen - und somit in Firmenbuch - aufscheint. Insgesamt gab es zu Beginn $20125 \mathrm{I} 4$ dieser Organisationen (Pennerstorfer et al. 2013) - die Anzahl von NPOs dürfte aber deutlich höher liegen.

Im Bereich der Genossenschaften finden wir m Jahr 20I0 I.8I7 Genossenschaften in Österreich (Statistik Austria 20I2), nur 95 davon
zeichnung 'gemeinnützig‘ im Namen.
BESCHÄFTIGTE

b im Jahr 20I0 Hochrechnungen zufolge ca. 2I 2.00o Vertragsverhältnisse im österreichischen Nonprofit Sektor, was einem Anteil von $5,2 \%$ aller in Erwerbstätigen über I5 Jahren entspricht. Davon entfiel mehr als ein Drittel (36\%) auf das Sozialwesen, weitere $20 \%$ auf den Bereich Interessensvertretungen sowie kirchliche und sonstige religiôse Vereinigungen`. Das Unterrichtswesen is der nach der Beschäftigung drittwichtigste Bereich Nonprofit Sektor. Zusäzlich abeiten ca. 2 Millionen Menschen freZillig in Sektor, ihre Arbeitsleistung im Rahmen

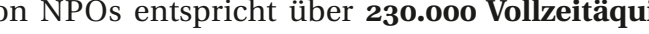

Auffallend ist die deutliche Zunahme der Vertragsverhältnisse. Ihre Anzahl stieg zwischen dem Jahr 2000 und 2010 um rund $39 \%{ }^{2}$ an, das Wachstum fand vor allem in den Branchen ,Kindergärten (93\% Zunahme) und Sozialwesen ( $76 \%$ ) statt, eine tor Unterhaltung statt (-26\%)

Die meisten Beschäftigten arbeiten im Bereich Krankenanstalten, Altenheime, Rettungsdienste, Interessensvertretungen organisationen und religiösen Vereinigungen, in weiterer weiterer gio

Auch hier ist die statistische Erfassung unzuichend. Eine Erfassung der NPOs und ihrer Beschäftigten entsprechend der Internationalen Klassifikation von NPOs - ICNPO (Meyer et al. 2010) würde die Rolle des Sektors besser verdeutlichen und internationale Vergleichbarkeit schaffen. In Österreich wird der Tätigkeitsbereich von NPOs nur nach ONACE erfasst, nach der statistischen SysteUnion.

Zwischen 2000 und 2010 hat sich der Anteil der Vertragsverhältnisse erhöht in den Bereichen Sozialwesen $(+7 \%)$, sowie Forschung, Entwicklung und Unterrichtswesen $(+1,8 \%)$, anteilsmäßig gesunken ist er in den Bereichen Interessensvertretungen und religiöse Vereinigungen $(-5,2 \%), \mathrm{Ge}$ sundheitswesen $(-2,8 \%)$, Unterhaltung $(-2,5 \%$

\section{FREIWILLIGES ENGAGEMEN}

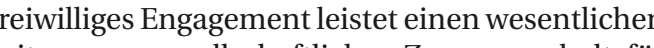
Integration und hicht zulaü die kostengüstige Erbringung

2006 waren $27.9 \%$ der Östrig

Rahmen von NPOs freiwillig tätig Sie leisteten dabei insgesamt 7.918.682 Stunden pro Woche (Daten aus diesem Kapitel: More-Hollerweger/Heimgartner 2009; More-Hollerweger/Rameder 2013).

Dies entspricht knapp 230.000 Vollzeitbeschäftigten oder aber $6 \%$ der unselbstständigen $\mathrm{Er}$ werbstätigen in Österreich. Der monetäre Wert der Freiwilligenarbeit ist ebenfalls hoch: Würde diese Arbeit zu Durchschnittslöhnen bezahlt, dann würde dies 4.727.I36.00o Euro kosten. Nach Berei- 
chen dominiert der Bereich der Katastrophenhilfe, der Kultur, der Religion und des Sports die Freiwilligenarbeit mit einer Beteiligungsquote von je $33,0 \%$, bei Frauen bei $23 \%$.

\section{EINNAHMEN UND AUSGABEN}

ie hochgerechneten Gesamteinnahmen der NPO betrugen 2005 rund 3,4 Milliarden Euro. Kranke hăuser und Schulen sind hier nicht enthalten. Die meisten Einnahmen beziehen österreichische NPOs chen Hand $(36 \%)$, gefolgt von Umsatzerlösen $(33 \%)$ und Subventionen (17\%), Spenden betragen $8 \%$ (Schneider/Haider 2009).

Insgesamt kommen also rund 53\% der Einnahmen von der öffentlichen Hand. $36 \%$ sin Markteinnahmen und i I\% Einnahmen aus Spo soring und Spenden.

Ein wesentlicher Faktor, der auch die Bedeutung von NPOs in den Augen der Bevölkerung zeig sind Spenden. Eine Erhebung dazu zeigt, dass in Unternehmen I50 Millionen Euro spendeten (Neumayr/Schober 20I2).

Die Ausgaben des österreichischen Nonprofit Sektors fur 2005 lion hochgect sonalausgaben mit über 2, Milliarden Euro einnehmen.

\section{LEISTUNGEN DES NONPROFIT SEKTORS \\ .Wertschöpfung}

ur Messung der wirtschaftlichen Leistungskra der NPOs ist die gesamtwirtschaftliche Wertschöpfung die beste Kennzahl. Dies ist der Wert, um den NPOs haben im Jahr 2010 5,9 Milliarden Euro Bruttowertschöpfung erwirtschaftet.

Die Berechnung stellt eine Untergrenze da da der Wert der ehrenamtlich geleisteten Arbe nicht einbezogen wird. In den letzten zeth ist die Wertschöpfung deutlich gestiegen.

Für den Bereich der Sozialwirtschaft, also Gesundheits-, Veterinär- und Sozialwesen, der aber auch die öffentlichen Einrichtungen umfasst, be rechneten Meyer/Neumayr einen Wertschöpfungsmultiplikator. Dieser ist wie folgt zu interpretieren: Die Erhöhung der Nachfrage nach ... um den Gesundheits- und Sozialbereich bedeutet das: Die Erhöhung der Nachfrage nach Dienstleistungen um I Mio. Euro löst eine Wertschöpfung von . für den Gesundheits- und Sozialbereich zeigt: Die Hï um I Mio. Euro benötigt I, 676 Mio. Euro an heimischen und importierten Gïtern.

Damit ist der Sozialbereich nach der öffentlichen Verwaltung und Landesverteidigung an zweitletzter Stelle. Er ist damit ein besonders nachhaltiger Bereich: Er schafft primär Arbeit, verbraucht dafür aber besonders wenig an Ressourcen bzw. rleistungen. Im Gegensatz dazu benötigt die chfrage um I Million Euro im Energiebereich Euro.

\section{b. Soziale Wirkungen des Sektors}

Der Sektor hat eine Fülle an sozialen Wirkungen. E erbringt Sozialleistungen, ermöglicht gesellschaftiche Vielfalt, Partizipation und Innovation - die meisten Innovationen im Sozialbereich sind im Rahmen von NPOs entstanden, auch die meisten nachst von Organisationen der Zivilgesellschaf aufgegriffen worden (Simsa 200I). Die meisten dieDen in keiner amtlichen Statistik.

Das Messen und Bewerten der Wirkungen von Nonprofit Organisationen gewinnt allerdings an Bedeutung. Damit haben in den letzten Jahren Analy sen des Social Return on Investment - SROI an Bedeutung gewonnen. Sie bieten eine sneue Kennzah den Sektor Die SROI-Analyse is in ih Spielart der Cost-Benast ist die Messung des Impacts, also die Grundidee in mit Geldwerten versehen. Ebenso werden die Inputs (verstanden als Investitionen) erfasst un bewertet. Der monetarisierte Impact wird nun in Relation zur Investition gesetzt (geobenenfalls abzüglich der Kapitalrendite für Investoren). Das Konzept des SROI zielt auf alle internen und externen Stakeholder ab, die durch die Aktivitäten posvoder auch negativ betroffen sind.

Der SROI eines Projekts ist somit eine Zahl, tete soziale Rückfluss sich aus einem in ein Projekt oder in eine Organisation investierten Euro rgibt (Schober et al. 2013).

Die Zahlen zum sozialen Rückfluss, die socherart bereits berechnet wurden, lassen sich nicht für den gesamten Sektor aggregieren. Sie zeigen abe sehr deutlich, dass sich Investitionen in NPO durchaus rechnen, vorsichtige Untersuchungen errechnen z. B. SROI-Werte in Höhe von 4,4I im Projekt starthilfe Wohnen (Rauscher/Pervan-Al So qauer 2012), bei der oberosterreichischen Feue(Schober et al. 2012) oder 3,4 hei der Casa Abraham.

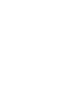

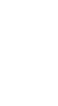

\section{AKTUELLE ENTWICKLUNGEN}

Engagement ist Österreich nicht gerade das aktivste Land. Verglichen mit den massiven Ausweitungen zivilgesellschaftlicher Proteste, Aktivismus und Selbstorganisation etwa in südeuropäischen Ländern, oder Bewegungen wie Occupy oder den Arabischen Frühling, ist es in Osterreich sehr ruhig. Bezogen auf die Rahmen von NPOs bietet sich ein anderes Bild. NPOs spielen eine zentrale Rolle in der österreichischen Gesellschaft. Die folgenden Entwicklungen konnen diesbezugich als zentral gesehen werden Meyer/Simsa 2013)

\section{a. Ökonomisierung}

Zivilgesellschaft ist zunehmend von Ökonomisierung, von einer sich in unterschiedlichen Facetten ausdrückenden Ubernahme der Logiken der Wirt schaft geprägt. Weiters scheint es, dass NPOs zu-
nehmend unter Generalverdacht geraten, sich vernehmend unter Generalverdacht geraten, sich ver-
mehrt legitimieren und beweisen müssen, effizient und wirtschaftlich zu handeln (Zimmer/Simsa und wirts.

Dorothea Greiling (2014) spricht vom Wandel von einer trust me- zu einer prove me-Kultur und Treitäts- und nelle Glaubwürdigkeitsbonus von NPOs ist demnach in den vergangenen Jahrzehnten sukzessive in Frage gestellt worden, Angesichts gegenwärtig deutlich werdender und mit Bedarf an massiver Unterstützung durch öffentliche Gelder einhergehender Misswirtschaft in zentralen Teilen des Wirtschaftssektors, ist es verwunderlich, dass gerade gemeinwohlorientierte Organisationen vermehrt ihre Berechtigung unter Beweis stellen müssen und dies noch dazu unter Rekurs auf Legitimationsmuster, die der Wirtschaft entstammen. In Osterreich gibt es gegenwartig im Zuge von Wirtschaftsforderung und Konsolidierungen massive stantiche Korrekturen der Märkte. Diese tragen nicht zur Entmystifizierung der neoliberalen Ideologie einer Glorifizierung von Mărkten, kurzfristigem Effizienzdenken und Engführung des Blicks auf rein ökonomische

In diesem Zusammenhang gewinnen die Themen Social Entrepreneurship, Wirkungsmessung und Philanthrokapitalismus an Bedeutung. Damit zeigt sich ein gewisses Verschwimmen der Grenzen duch tepte Public Private Partnerships und Venture PhilanthPop Dies gleichzeitig aber stärker an gesellschaftlichen zen orientiert als klassische Profitunternehmen. Treiber hinter dieser Entwicklung sind vielfach private PhilanthropInnen, Stiftungen, Impact Funds oder gesellschaftlich verantwortungsvolle Unternehmen: Sie investieren lieber in Organisationen die stärker markt- und auch gewinnorientiert agieren als in klassische NPOs.
Hintergrund dieser Entwicklung ist der aube, dass erwerbswirtschaftliche Handlungsmuster auch in sozialen und ökologischen Belangen den nicht-kommerziellen bzw. zivilgesellschaftlichen deutlich überlegen sind. In dem Zusammenhang steht auch die Forderung nach weitergehendem Managerialismus, d. h. nach weiterer Ubernahme von Tools aus der Betriebswirtschaft durch NPOs. Dadurch sind sicher noch vererrelt Efizienzgewinne von NPOs erreichbar. Im Sinn einer nachhaltigen okonomischen und sozialen Entwicklung wäre aber auf gesellschaftlicher Ebere eine stankere Orientierung an jenen Werten, die tendenziell der Zivilgesellschaft und NPOs zugeschrieben werden, jedenfalls hilfreicher als weitere einseitige Ökonomisierung.

\section{. Nonprofits and the government}

\section{More Work for Less?}

Der offentliche Sektor ist in vielen Bereichen wichigster Auftrag- und Geldgeber für NPOs. Damit . c Managenent Konzepten, die au Zielverinbrungen und Leistungsverträge zweitens von der angespannten La öffentlicher . ders kritisch auf der Ebene der Kommunen.

Contracting Out bedeutet die Übertragung öfentlicher Aufgaben an NPOs auf Vertragsbasis, also auf Basis von Leistungsverträgen zwischen einer PO und der öffentlichen Hand. Die Beziehung ungs-Gegenleistungs-Basis gestellt, was einen bedeutenden Unterschied gegenüber einer Fördeung der NPO durch Subventionen macht. So führt es zu spezifischen Problemstellungen für das Magement einer NPO und hat daruber hinaus eine

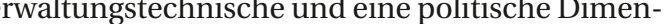
ton. Es kann eine Anderung der Aufgabenwahr-

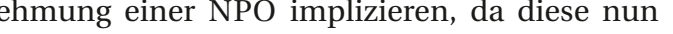

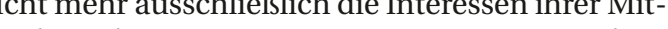
ensumentinnen zu vertreten hat, 列

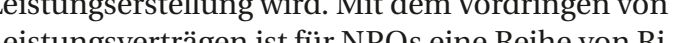

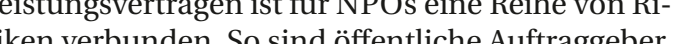
ff nicht in n durchzuführen, die tsä̈hlich

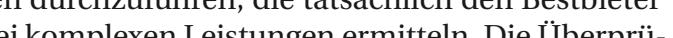
fung der sich NPOs durch die öffentlichen Avtr hen lossen müssen, reduziert sich auf Finanzierungs- und Angebotsebene. Output d nicht Outcome wird gemessen, oftmals werden er Bestbietern bevorzugt.

Die Finanzkrise öffentlicher Haushalte war chon vor der 2008 einsetzenden Wirtschaftskrise eine permanente Herausforderung für NPOs. Beiglich der Finanzierung durch die öffentliche Entwicklo es in den letzten Jahren eine eindeutige des Sektors stehen gleichbleibende bzw. rückläufige Finanzierungen durch die öffentliche Hand gegen- 
über. Zusätzlich werden Zahlungen bzw. Vertragsniger plentlich ungewisser, kurzfristiger und we 20r planbar (Meyer/Simsa 2013)

Der Hintergrund ist der Trend zur Deregulierung und Privatisierung sozialer Aufgaben. Die neoliberale Ideologie plädiert für eine radikale Deregulierung: Arbeit, Soziales und Demokratie werden damit der dominanten Logik der Wirtschaft untergeordnet, und der Staat soll grundsätzlich möglichst wenig in die Gesellschaft eingreifen. Die Sozialquoten, also der Anteil der Sozialausgaben am BIP, sind in der Folge trotz krisenbedingt höheren Beblieben bzw. sogar leicht gesunken: In Österreich sank die Sozialquote von $26,7 \%$ (2000) auf $26,4 \%$ (2010) (OECD 2010)

Ökonomische Analysen zeigen eine Umverteilung von Unten nach Oben, und die Kluft zwschen Reich und Arm hat sich in den letzten Jahren
drastisch verschärft (Marterbauer 20II). Die Wirtschaftskrise hat bereits zu einer Verschärfung der schaftskrise hat bereits zu einer Verschartung der
sozialen Unsicherheiten geführt, die Entwicklung sozialen Unsicherheiten gefuhrt, die Entwicklung
zu Prekarisierung, einer zunehmenden Kluft zwischen arm und reich und wachsender Exklusion is noch nicht beendet und die Frage nach gesellschaftlicher Integration und sozialer Gerechtigkeit stellt sich damit in verschärfter Form. Wohlgahrtsstatliche Absicherung und Solidarität verlieren also an Bedeutung zugunsten von Eigenverantwortung und privatem Engagement.

\section{c. Neue Betätigungsfelder durch}

neue Anforderungen

Die Entwicklung des Sektors wurde in den letzten Jahren durch neue Aufgabenfelder bzw. neue Ausprägungen von Tätigkeiten innerhalb eines Arbeitsfeldes geprägt. Umwelt und OKologie, Pflege un Altern, Migration, Gesundheit, Soziale Ungleichhei Jugend und Bildung haben in den letzten Jahren steigende Bedeutung erfahren, ein Trend, der sic vermulich fortsetzten wird, insbesondere in Bezug auf den Bereich Pflege und Altern, eine Einschäzung, die durch demographische Prognosen untermauert wird (European Commission, 2009).

Generell zeichnen sich zwei Entwicklungen ab: Auf der einen Seite werden Anforderungen an die Leistungen vieler NPOs höher. KientInnen oder ihre Angehorigen werden informierter und anspruchsvoller, sie erwarten hochprofessionelle, maßseschneidete und modene Angebote. Gleichzeitg entsteht in rolge der wachsenden sozialen rellen Arbertosing Klientel für NPOs. Auch die Einschrönkung sertlicher Infrastruk tur Auch dic Handung felder und Klientele von NPOsvë̈nden.

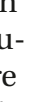
gement ist bereits vielfältiger geworden, projektfö̈miger, weniger stabil und wird verstärkt in Zusammenhang mit persönlichen Entwicklungszielen oder beruflichen Zielen gesehen. Neue Formen wie episodisches oder virtuelles Volunteering, online Volunteering und selbstorientiertes Volunteering werden in Zukunft vermutlich an Bedeutung gewinnen. Damit verandert sich nicht nur die Arbeit und organisationale Anbindung der Freiwilligen, sondern es entstehen auch neue Anforderungen an deren Management bzw. generell an NPOs. Es geh also darum, von Seiten der NPOs Gelegenheiten zu bieten, die den Ansprüchen potenzieller Freiwilliger an Selbstentfaltung, sporadisches Engagement und der Erhohung beruflicher Chancen gerecht werden und es wird verstarkt auch darum gehen, die dami einhergehende hônere Flexibilitát und Fluktuation organisatorisch zu be (neger 2009).

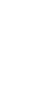

r-
. end, üt.

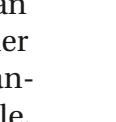


ANALYSE DER RAHMENBEDINGUNGEN FÜR DIE ZIVILGESELLSCHAFT

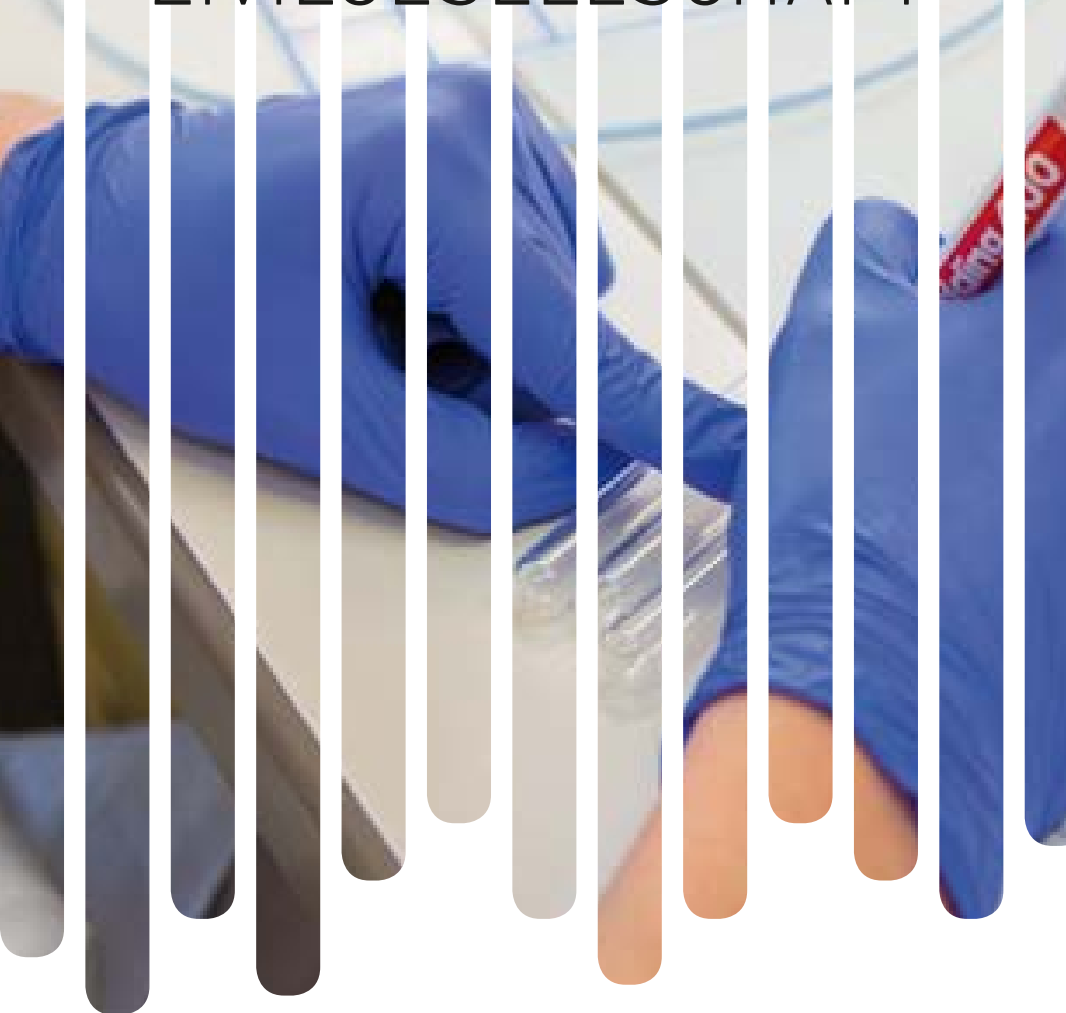

1. Demokratie in Österreich

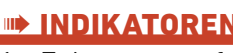

Existenz von freien und fairen Wahlen

2. Existenz von zielgruppenspezifischer Repräsentanz $\frac{\text { (z. B. Kinderparlament, Stadtteilparlament) }}{3 . \text { Existenz von Formen der direkten Demokratie }}$

(Volksbegehren, Volksabstimmung)

4. Wahlbeteiligung - aktives und passives Wahlrecht

politischer Partizipation

6. Die Expertise der CSOs wird beratend in Bezug auf Aktivi-

7. Level der Ex politischer Tonutert (z. B. M

politischem Konflikt

8. Ausmaß der Trennung der Gewalten (Exekutive/Legisla-

tive/ Judikative

\section{DAS POLITISCHE SYSTEM ÖSTERREICHS}

Die Republik Österreich ist ein Bundesstaat besteRend aus neun Bundesländern und wurde 1918 als auf der 1945 wiedereingesetzten Bundesverfassung von I920. Betrachtet man alleine die Verfassung mit dem/der direkt gewählten österreichischen Bundespräsidenten oder -präsidentin und seine/ihre Vielzahl von Machtkompetenzen sollte das politische System Österreichs als semi-präsidentiell eingeordnet werden. Jedoch verzichtet der/die österreichische Prasidentin traditionell auf seine/ih starke Rolle (Helms/Wineroither 2012). Die Regierung besteht deshalb aus dem/der durch das Parlament legitimierte/ $\mathrm{n}$ BundeskanzlerIn und den Ministermnnen. Verfassungsrealistisch ist Osterreich gewählten parlamentarischen Kammer, dem Nationalrat, und einer zweiten parlamentarischen Kammer, dem Bundesrat, dessen Mitglieder übe die neun Landesregierungen bestellt werden. Die Abgeordneten des Nationalrates werden in einem Verhältniswahlsystem von allen BürgerInnen übe I6 Jahre gewählt. 1992 wurde das Verhältniswahlrecht personalisiert und beinhaltet nun die Möglichkelt der Vergabe un Vorzugsstimmen auf Regonalwe Lan im Jahr 2007 womit Österreich als einzigen isches 2007 wonit Ostend anziges europaisches Land das generelle Wahlalter auf I6 Jahre gementsmehrheit bestimmt nach der Die ParlaRegierung, welche in der zweiten Republik meh heitlich aus großen Koalitionen der zwei Volksparteien sPö und övp bestand. Formell werden $\mathrm{der} /$ die BundeskanzlerIn und die Regierungsmitglieder von der Bundespräsidentin/dem Bundespräsidenten ernannt, jedoch kann der Nationalrat die gesamte Regierung und auch einzelne Mitglieder absetzen, dadurch besitzt er die endgültige politische Kontrolle über die Regierung. Die höchste juristische Kontrolle besitzt der Verfassungsgerichtshof mit der Kompetenz der Normenkontrolle von Gesetzen und Verordnungen
DIE DEMOKRATISCHE QUALITÄT ÖSTERREICHS

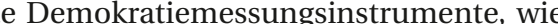
der ,Freedom in the world--Index von Freedom House und das 'Polity IV Project v von Monty G. Mahrshall und Ted Robert Gurr, stufen Osterreich als etablierte Demokratie ein. Im Polity Project, welches sich auf die verfassungsrechtliche Verankerung der Demokratie und auf die demokratische Qualität der Exekutive fokussiert, ist Osterreich seit dem Beginn der zweiten Republik eine ,vollständige Demokratie. Der Index hebt insbesondere formale Demokratieaspekte, wie die Kontrolle der Exekutive und de Östbewerb beider Besetzung hervor, wel-

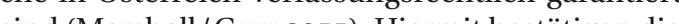
Autoren, dass die östricichische Verassung alle wichtigen denobatischen Aspeke Freedom in the wold I dex hingegen betacter die politischen und die bürgerlichen Feiter Österreich erreicht seit dem Beginn der Messung I972 immer die volle Punktzahl in beiden Dimensionen und gilt deshalb als frei (Freedom House 20I b; c) Der Status frei bedeutet dabei, dass Österreich ein kompetitives Mehrparteiensystem und allgemeines Wahlrecht für alle erwachsenen BürgerInnen besitzt, dass Wahlen regelmäßig und ohne Betrug durchgeführt werden, dass diese repräsentativ die öffentliche Meinung abbilden und dass die Möglichkeit gegeben ist das Elektorat offen über die Medien und politischen Kampagnen anzusprechen (Campbell/Barth 2009). Insgesamt kann gesagt werden, dass Österreich mehr als die demokrati- schen Minimalkriterien erfültt und seit dem Beginn der zweiten Republik als eine stabile und etablierte Demokratie gilt.

Die genannten Indizes schaffen einen guten entren Eindruck. Da ihre primäre Funktion jedoch der Abgrenzung zwischen Demokratien und $\mathrm{Au}$ tion darüber, wie die Österreichische Demokratie ausgestaltet ist. In den nächsten Abschnitten wird die demokratische Qualität Osterreichs an Hand von ausgewăhlten Aspekten näher betrachten. Die Darstellung folgt den Dimensionen der demokratschen Qualitat nach Hans Joachim Lauth: Freiheit, Gleichheit und Kontrolle (Lauth 2004). Abbildung stellt die Ergebnisse der Messung der demokrat schen Qualităt durch das Democracy Barometer da. Das Democracy Barometer vergleicht dreißig etablierte Demokratien an Hand der drei Dimensionen nach Lauth und je drei weiteren Subdimensionen: Individuelle Freiheiten, Rechtsstaatlichkeit und Offentlichkeit konstituieren die Dimension der Freiheit, Weitbewer, Gewaltenkontolle und Regie-

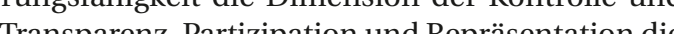
Dimension der Gleichheit. Gemessen waton die Subdimen bei die Skala relativ ist und roo die höchste empirische Ausprägung und o die niedrigste empirische Ausprägung darstellt.

mocracy-Barometer: demokratische Qualität Österreichs

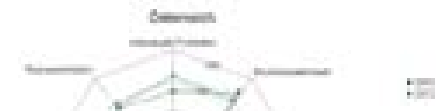

-

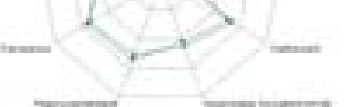

ocracy Barometer 20

Die Stärken der österreichischen Demokratie liegen in der Garantie auf die Auslebung der indivduellen Freiheiten und der Rechtsstaatlichkeit. Hingegen ist die Starke der Partizipation nur durchchnittlich und die Performanz in der offentlichen Sphäe beunruhigen. Die niedrige Ausprägung er-

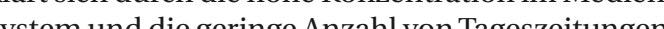
(Demorientration wird von mehreren AutorInen äs überoch eingestuft und insbesondere die Dominanz der Neuen Kronen Zeitung als beunuhigend für die demokratische Qualität gesehen Krabb 20I I; Freedom House 20I4a).

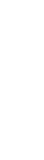

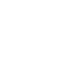




\section{A POLITISCHE FREIHEIT}

Wie schon vom Democracy Barometer dargestellt

(7) ist die Dimension der Freiheit mit Ausnahme der Medienkonzentration in Österreich überdurchschnittlich hoch. Insbesondere in der Rechtsstaa-
lichkeit hat Österreich hohe Werte. Rechtsstaatlichkeit beinhaltet ein effektives Justizsystem, Vertrauen der Gesellschaft in das Justizsystem und Gleichheit vor dem Gesetz. Die österreichischen BürgerInnen haben großes Vertrauen in die Justiz, in die Verwaltung und in die Polizei (Plasser/Seeber 2012). Eine besondere Institution ist die 1977 eingeführte Volksanwaltschaft, welche I982 verfassungsrechtlich verankert wurde (Ucakar/Gschiegl 2009). Die Volksanwaltschaft hat zwar keine effektive Entschedungsgewalt ist aber eine weitere rechtliche Kontrollinstitution und agiert als Ombudsorgan für alle BürgerInnen im Umgang mit der offentlichen Verwaltung. Damit wurde ein Instrument geschaffen das außerhalb der regularen Justizsystems die BürgerInnen vor der Willkür des Staates beschützt und Freiheitsrechte garantiert.

Eine freie öffentliche Sphäre und ein pluralisGründen wichtig für das Funktioniectiener mokratie Die Medien agieren als Kontrollinstanz der staatlichen Institutionen, aber auch als Informationsquelle der BürgerInnen für die Participation im politischen System. Damit schaffen sie die öffentliche Sphäre die es zum Ausleben der politischen Rechte benötigt. Deshalb sind die konzentrierten Besitzverhältnisse der Medien in Österreich, insbesondere bei Printmedien, als kritisch einzustufen. Dennoch haben die österreichischen Medien gerade in der Aufdeckung von Korruptionsfällen in den letzten Jahren ihre Kontrollfunktion gut erfüllt (Krabb 20I I). Der öffentliche rechtliche Rundfunk ORF ist gesetzlich der Objektivität und der Unparteilichkeit verpflichtet (vgl. ORF 2012 \$ I (3) \& \$ Io(5) ORF-G) ${ }^{3}$ und umgeht damit die negativen Konsequenzen der Medienkonzentration in Österreich. In der Praxis wird gerade bei der Besetzung von höheren Positionen im ORF parteipolitischer Einfluss vorgeworfen, wie bei der Bestellung der Büroleitung der Generaldirektion. Bemerkenswert war dabei die Reaktion der RedakteurInnen, die sich gegen den parteipolitischen Einfluss öffent lich gewehrt haben, was den Rückzug des Bewerbers zur Folge hatte. Generell ist die Auslebung der die demigkeit der Medïn ausschlaggebend fur die demokratische Qualitat der offentichen Sphäre Parteieinfluss erschwert wird.

Obwohl Österreich insgesamt hohe Werte für die Rechtsstaatlichkeit aufweist, ist im Hinblick auf die rechtliche Sicherung der politischen Freiheiten und der politischen Partizipation der $\$ 278$ des StGB bedenklich (siehe auch Kapitel II/3). Die breite Formulierung einer kriminellen Organisation und deren Tatbestand schaffen Auslegungsspielraum, welcher Ermittlungen gegen die kritische Zivilgesellschaft ermöglicht. Kritik an der Auslegung des Paragraphen, welcher zur Anklage von I3 TierschutzaktivistInnen führte, kam von einer Vielzahl von NGOs, PolitikerInnen und auch RechtswissenschaftlerInnen (Reindl-Krauskopf/Salimi 20I I).

\section{9}

len Organisationen ‘ bei einem Straftatverdacht prä- an politischen Prozessen beteiligen. Nach Verba et al. ventiv ermittelt werden kann, führte zu der breiten Überwachung der TierschutzaktivistInnen. Dies kann als Instrument gegen die kritische Zivilgesellschaft verwendet werden (Amnesty Internationa 20134). Das Bestreben der Regierung, weitere Kompetenzen zur Terrorismusprävention zu schaffe führte zu vergleichbaren Reaktionen der Zivilgesel schaft. Der Straftatbestand im Gesetzesentwurf war ähnlich breit formuliert und ließ damit Spielraum zu, der zur Anklage von NGOs und auch Journalisten hätte führen können (vgl. Reporter ohne Grenlität hat die Regierung auf die demokratische QuaStrafgesetzbuches die mögliche Einschränkung des Strafgesetzbuches die mögliche Einschränkung der Zivilgesellschaft durch $\$ 278$ a verkleinert (vgl. BGBl. I Nr. I34/20I3), jedoch muss kritisch beobachtet
werden ob neue Gesetze zurTerrorismusprävention die öffentliche Sphäre zu Gunsten von mehr Sicherheit einschränken (siehe auch Kapitel III/2-3) Ähnliches Missbrauchspotenzial wird in Bezug auf den $\$ 274$ StGB Landfriedensbruch gesehen, wie in Kapitel III/3 näher erläutert.

\section{GLEICHHEIT UND PARTIZIPATIONSFORMEN}

Im politischen Prozess müssen alle BürgerInnen gleiche Partizipationstechte besitzen, um effekt Österreich werden die I83 Abgeordneten des Nationalrates alle fünf Jahre gewählt. Dabei gelten das passive Wahlrecht von I6 Jahren und das aktive von I8 Jahren. Im europäischen Vergleich ist Österreich mit dem Wahlrecht aller BürgerInnen ab I6 Jahren progressiv. Das österreichische Wahlsystem ermög-
licht einen freien Wettbewerb um WählerInnenstimmen und garantiert die notwendigen Bürgerrechte für einen fairen Wettbewerb (Pelinka 20I I). Für österreichische StaatsbürgerInnen sind die Wahlen in Österreich als fair und frei zu bezeichnen. Problematisch ist jedoch, dass $9 \%$ der in Österreich lebenden Personen auf Grund ihrer nichtösterreichischen Staatsbürgerschaft von Wahlen
ausgeschlossen sind (Krabb 20II). Der Großteil dieausgeschlossen sind (Krabb 20I I). Der Großteil dieser Bevölkerungsgruppe besitzt keine Staatsbürgerschaft eines anderen EU Landes und darf deshalb auch nicht bei Kommunalwahlen und Wahlen für
das Europäische Parlament teilnehmen. Die in Ösdas Europäische Parlament teilnehmen. Die in Osterreich legal lebenden AusländerInnen haben die
gleichen Verpflichtungen wie InländerInnen, jegleichen Verpflichtungen wie Inländerlnnen, je-
doch wenige politische Rechte. Bezieht man Campdoch wenige politische Rechte. Bezieht man Camp-
bells (2012) Ergebnisse mit ein, dass es in Österreich bells (20I2) Ergebnisse mit ein, dass es in Osterreich erlangen, ist die Exklusivität des Wahlrechts als de mokratisch beunruhigend zu betrachten.

Neben formalen Ausschlusskriterien gibt es eine Reihe von anderen Einflussfaktoren, die bestimmen, ob und in welcher Form sich BürgerInne

hittp: / / www.amnesty.at/service_links/presse/pressemit-
teilungen/amnesty_international_und_greenpeace_fordern_ reform_des_

http:// /www.rog. at/2 278stgo-untergrabt-demokratische-
(I995) zählen insbesc wie der sozioökonomische Hintergrund (Bildung Beruf, Einkommen), Sozialisation durch Familie, also Netzwerke, die Individuen zur Beteiligung motivieren, zu den wichtigsten Determinanten, die Osterreich ist die Datenlage zur politischen Beteiligung von Bürgerlnnen generell eher spärlich, es gib einzelne Analysen, die Zusammenhänge zwischen sozialer Ungleichheit und politischer Partizipation Die (z. B. Matzinger 2012, Walter 2012). it Die Sozialpartnerschaft, als weitere Moglich(ie Ausgestaltung der Politik zu mokrati hat in der osterreichischen Konkordanzdeund ArbeitnehmerInneninteressensorganisationen sowie wie die Kammern, hatten großen Einfluss auf die Willensbildung im vorparlamentarischen Raum. Thr Einfluss hat besonders mit der ÖVP/FPÖ/BZÖ Koalitionsregierung abgenommen, jedoch wurde me

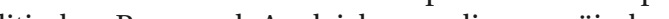

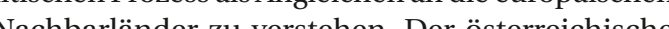
Korporatismus war ein internationar Sondefall ist heute jedoch ein normarer Fall von Interessensvertretung. Der Rückgang erhöht die demokratische parlamentarischen Raum getätigt werden und die Besetzung von Ämtern transparenter geschieht (Krabb 20II)

Die osterreichische Verfassung garantiert eine Bundesebene und ist damit im europäischen Ver. Volksbegehren haben die BürgerInnen ein direktes Pestre ein Volksbegehren von mehr als 100.000 Personen unterzeichnet, muss der gedecter dele werkech kein Recht auf eine Entscheidung im Nationalrat.

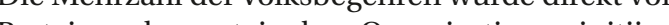
Parteien odor parteinahen Organisationen initiiert und Seer Instrument, das zund deshab Volksbegehren ein

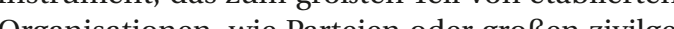
Orger Ressoucen genutzt wird Von den 37 Volksbegehren in der zweiten Republik wurden bislang nur$$
\text { griffen. }
$$

In der Praxis ist dadurch das Initiativrech durch die Bevölkerung eingeschränkt. Zivilgesellschaftliche Initiativen fordern seit Jahren das Instrument Volksbegehren zu reformieren, wie zum Jetzt! ${ }^{6}$ oder durch den offenen Brief für ein offenes Demokratiereform-Büro im Nationalrat. Ende $2012^{7}$ Zivilgesellschaft auf und einigte sich auf ein Demokratiepaket, welches das Volksbegehren aufwerten

http://www.bmi.gv.at/cms/BMI_wahlen/volksbegehren/vb_ schule und Arbeitsplatz sowie das soziale Umfeld, auch empirisch schon vielfach bestätigt wurden. In der Korporatis Koalition 2006 Qualität, weil dadurch weniger Tauschakte im vorReihe von direktdemokratischen Instrumenten auf nahm die Regierung Teile der Forderungen aus der 


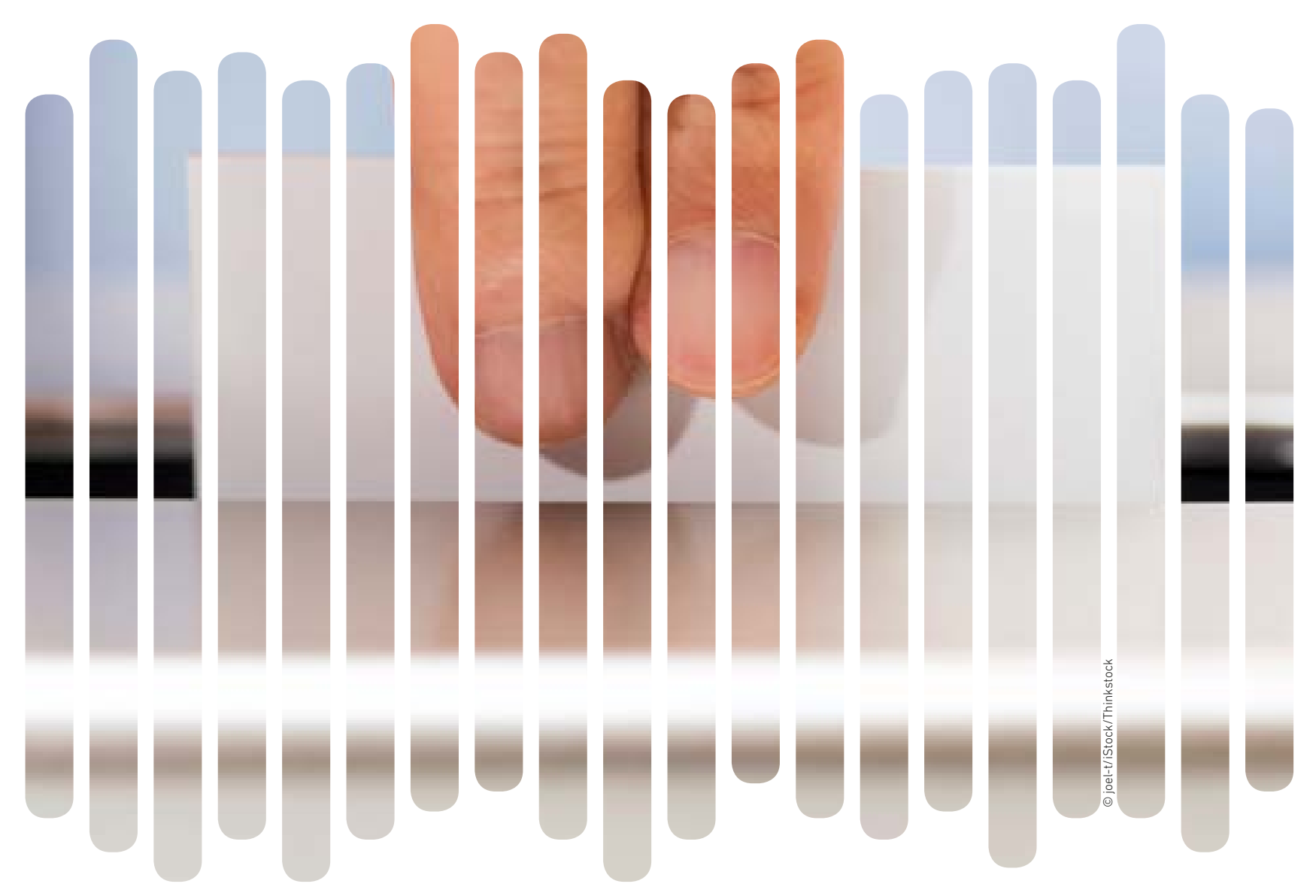

sollte, jedoch wurde nach einer kritischen Stellung- stellt werden, welche in Bundeszuständigkeit fallen. nahme des Verfassungsgerichtshofes im August Sie können entweder von Nationalratsmitgliedern 2013 die Reform verschoben ${ }^{8}$ Insgesamt zeigt die vorgelegt werden oder direkt mit mindestens 500 Regierung durch Vorschläge Interesse an der Auf- Unterschriften von österreichischen Staatsbürgewertung des Volksbegehrens, doch ihre Vorschläge $\quad$ rInnen über I9 Jahre. Mit der Möglichkeit der online wurden bis jetzt noch nicht umgesetzt.

Ein starkeres Instrument ist die Volksbef

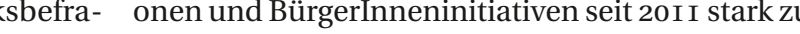

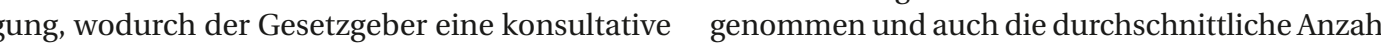
Meinungs- bzw. Willensäußerung einholen kann. Obwohl das Instrument schon seit 1988 verfassungsrechtlich verankert ist wurde es bundesweit 2013 zum ersten Mal für die Volksbefragung zur Wehrpflicht verwendet. Im Gegensatz zur Volksbe-
fragung sowie zum Volksbegehren ist das Ergebnis fragung sowie zum Volksbegehren ist das Ergebnis
einer Volksabstimmung bindend. In Österreich einer Volksabstimmung bindend. In Österreich
wurden bis jetzt zwei Volksabstimmungen durchgewurden bis jetzt zwei Volksabstimmungen durchgefuhrt. Eine fakultative Volksabstimmung im Jahr 1978 zur Nutzung Kencherie und 1994 ein obObligatorische Volksabstimmungen sind bei Verfassungänderungen festgeschrieben und verk̈̈rperdic Rolle des Volkes als endgültiger Souverän BürgerInneninitiativen und Petitionen haben in Österreich eine lange Tradition, wobei die Kriterien bis I988 restriktiv geregelt waren. Petitionen und BürgerInneninitiativen können zu allen Themen ge-

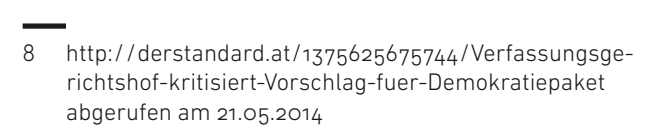

Buerger-ins-Parlament-schaffen abgerufen am 21.05.2014

https://charta.wien.gvat//start/charta/ abgerufen am verarbeitet, welcher die partizipative Erarbeitung werden (Parteiengesetz 20I2, S. \$ 6 [4] [5]). Damit von Grundsätzen und Spielregeln für ein gutes Zusammenleben darstellt. Die Bedeutung des Internets für partizipative Projekte, Petitionen und BürgerInneninitiativen hat in den letzten Jahren stark zugenommen. Erfahrungen aus diesen lokalen alternativen Repräsentationsformen könnten für die Bundesebene verwendet werden und zeigen in Kleinen die politischen Möglichkeiten auf.

uf Bundesebene existieren insbesondere in Bundesministerium für Arbeit und Soziales (BMASK) und im Bundesministerium für Land- und Forstwirtschaft, Umwelt und Wasserwirtschaft (BMLFUW) Strukturen, um zivilgesellschaftliche Organisationen einzubinden. Das BMLFUW hat eine
Reihe von Dialogforen, regelmäßige InformationsReihe von Dialogforen, regelmäßige Informationsveranstaltungen und den Klimaschutzbeirat einge-
richtet, in welchen zivilgesellschaftliche Organis richtet, in welchen zivilgesellschaftliche Organisationen zur Beteiligung in der Maßnahmenentwicklung und in themenspezifischen Stakeholder-Dialogen eingebunden werden (Oberhuber et al. 20I2)

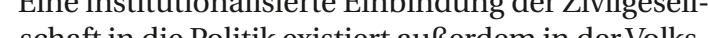
schwerts Beint untersuitzt und ber̈t die Arbeit derVolksenwaltschaftund ihre Kom Aufgaben im Bereich Menschenrechte. Die Volksanwaltschaft überprüft in Österreich die öffentlicheund privaten Finrichtungen in denen Freiheitsbeschränkungen durchgeführt oder vermutet werden. Dabei ist der Beirat in alle Gebiete der Prüfungstäti Dabei ist der Beirat in alle Gebiete der Prüfungstätigkeiten eingebunden, und wird somit bereits bei de tig. Die Kompetenzen des Beirates erstrecken sich tig. Die Kompetenzen des Beirates erstrecken sich
durch sein Anhörungsrecht auch auf die Bestellung von Kommissionsmitgliedern und -leitungen. Der
Beirat ist paritätisch besetzt durch VertreterInnen der zivilgesellschaftlichen Organisationen im Bereich Menschenrechte und VertreterInnen der betroffenen Ministerien inklusive LandesvertreterInnen ${ }^{11}$.

Jedoch sind Projekte dieser Art noch wenig in der Öffentlichkeit bekannt und aus der Perspektive der Zivilgesellschaft noch zu gering an der Zahl. Gemeinsam haben I5 NGOs im Oktober 20I3 gefordert, dass die Zivilgesellschaft stärker in de Politikprozess eingebunden wird ${ }^{\mathrm{I}}$. Dies zeigt, dass,
obwohl einige Ministerien die Zivilgesellschaft stärobwohl einige Ministerien die Zivilgesellschaft stärker in ihre Arbeit einbinden, noch viel Spielraum für
eine stärkere Kooperation existiert und für eine grö-

Dies wïrde insber

Österreich fördern, welche die Regierung 2012 in einem anderen Aspekt gestärkt hat. Bis zum Jahr 2012 waren Parteien nicht verpflichtet, die Namen der SpenderInnen und die Höhe der Spenden zu ver̈ffentlichen, sondern nur die gesamte Summe an Spenden. Mit der Änderung des Parteiengesetzes im Jahr 2012 hat die Regierung die Spendentransp renz erhöht. Nun müssen alle SpenderInnen, deren Zuwendungen in einem Kalenderjahr 3.500 Euro überschreiten, namentlich genannt und Spenden über 50.0oo Euro sofort dem Rechnungshof gemeldet werden, welche dann mit Namen und Anschrift auf der Website des Rechnungshofes veröffentlicht

http:// volksanwaltschaft.gv.at/mens
rechtsbeirat abgerufen am 05.05.2014

12 http:// www.amnesty.at/service_links/presse//pressemit-

schaft_staerken/ abgerufen am 05.05.20
schen Osterreich die mögliche Einflussnahme vo GoßsspenderInnen transparent gemacht und die terreich im internationalen Vergleich im Bereich der Transparenz im oberen Mittelfeld (vgl. Democracy Barometer 2013). Transparenz ist eine grundlegende Voraussetzung für die vertikale und horizontale Rechenschaftspflicht der Politik und deren Volk. Eine Erhohung der Transparenz, insbesondere bei der Finanzierung von Parteien und politischen -

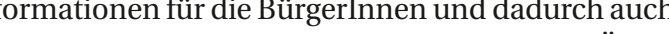
die demokratische Qualität eines Landes. Osteren den Politikprozess einbinden und 作 entgegen wirken, wie dies auch im Zuge des geplan

Beispiele, wie der JungbürgerInnen-Rat Wind haag ${ }^{\mathrm{I}}$ zeigen, dass Partizipationsprojekte nicht nur werden Region gemeinsam mit der Gemeinde einen (n) in 作 ben in Windh a betreffend wichtig ist Durch Projekte, wie dieses, ist es möglich, die Meinung der lokalen Bevölkerung und Gruppen, wie zum Beispiel Jugendlichen, in Entscheidungsprozesse und in die Gemeindeentwicklung einzubeziehen. Es existiert eine Vielzahl von ähnlichen Projekten, wie z. B. das Kinderparlament in Graz, in welchem Kindern Politik näher gebracht wird, gleichzeitig erlangen PolitikerInnen Verständnis für die Interessen der Kinder. Eine wichtige Voraussetzung für aktive Statsburgermnen ist das Wissen über das eigene politische System und über die eigenen politischen Rechte. Das osterreichische Parlament realisierte zu . statt und das Jugendparlament, in welchen zwe pro Jahr Schulklassen der neunten Schulstufe . 列 durger.

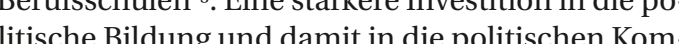
Österreichs stärken.

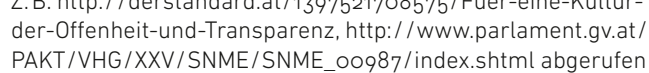
am 19.05.2014

thp.//Tww.patizipation.at/fileadmin/media_data/Downloads/Praxisbeispiele/praxisbeispiel_jugendrat_windh
fuer_partizipation_. pdf abgerufen am 05.05.2014

http://www.igpb.at/Pos
Schulisch_26.2.2010.pd petenzen der Bürom 


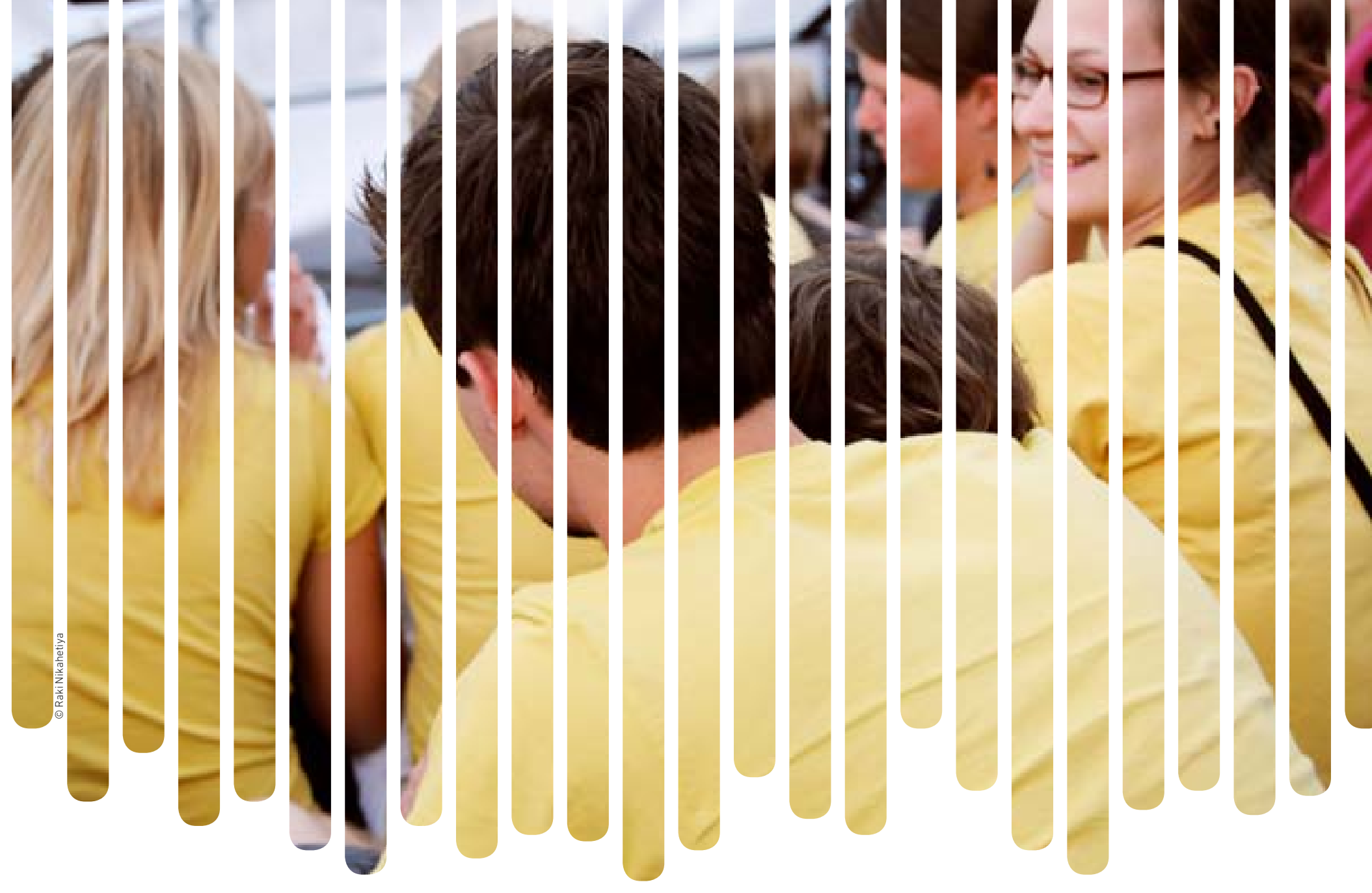

2. Vereinigungsfreiheit

ie Vereinigungsfreiheit bildet eine wichtige Grundlage für die Zivilgesellschaft, da sie BürgerInnen das Recht zusichert, Vereinigungen, etwa in Form von Vereinen, Stiftungen und anderen Arten von Nonprofit- bzw. Nichtregierungsorganisationen zu bilden. Sie ist, gemeinsam mit der Versammlungsfreiheit, in der Europäischen Menschenrechtskonvention (EMRK) Artikel I I Absatz I verankert: Alle Menschen haben das Recht, sich friedlich zu versammeln und sich frei mit anderen zusammenzuschließen, einschließlich des Rechts, zum Schutze ihrer Interessen Gewerkschaften zu bilden und diesen beizutreten..$^{16}$ Die Vereinigungsfreiheit
Österreich verfassungsrechtlichen Status.

Im internationalen Vergleich schneidet Österreich sehr gut ab (Freedom House 2013). ${ }^{17}$ Nichtre gis einwandfrei entwickett und di Menschen Bürgerrechte werden von vielen österreichischen und internation NGOs und Behörden geschützt (US Department of Stase 20I2) ${ }^{18}$ In diesem $A b-$ schnitt wird der Frage nachgegangen, was die Besonderheiten für CSOs in Bezug auf die Vereinigungsfreiheit in Österreich sind.

$\overline{16}$

https:/ / www.ris.bka.gvatat/GeltendeFassung. wxe?Abfrage=-
Bundesnormen\&Gesetzesnummer=10000308 abgerufen am

P.

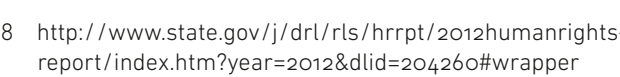

RECHTLICHE REGELUNGEN

RECHTLICHE REGELUNGEN-INDIKATOREN

Stiftungen und andere Arten von Nonprofit- bzw. Nicht-

regierungsorganisationen für jeden Zweck gründen kann.

rung (Alter, Nationalität, Rechtsfähigkeit, Geschlecht usw.)

auszuüben.

denen Organisationen entscheiden, sich zu registrieren,

ermöglichen eine einfache, zeitnahe und preiswerte Regist-

ein Berufungsverfahren

Das Gesetz erlaubt die Vernetzung zwischen Organisationen

ie rechtlichen Regelungen in der österreichischen

Verfassung gewährleisten das Recht auf Ausübung

ezieht sich auf natüliche und juristische Perso-

nen. Bei einem Verein schließen sich zumindest

zwei natürliche Personen zusammen um einen ge-

meinsamen ideellen Zweck zu verfolgen. Der Sitz

des Vereins muss im Inland liegen. Nach dem gel-

tenden Vereinsgesetz darf ein Verein nicht auf Ge-

winn ausgerichtet sein und das Vereinsvermögen

darf nur im Sinne des Vereinszwecks verwendet

abgerufen am 17.03.2014 
werden (Vereinsgesetz 2002 -VerG) ${ }^{19}$ Strebt ein Ver- des Vereins nicht gestattet wird. Gegen diesen Beein die Gemeinnützigkeit und die damit einherge-
hende steuerliche Begünstigung kann berufen werden. Wenn die Vereinsbedesabgabenordnung (BAO) an, muss in den denken gegen eine Vereinsgründung hat und eine Statuten u. a. sichergestellt sein, dass das Vereins- negative Erklärung ins Auge fasst, muss sie den An vermögen auch im Fall seiner Auflösung ausschließ- zeigern der Vereinserrichtung aber vorher Gelegen$\begin{array}{ll}\text { lich für gemeinnützige Zwecke verwendet werden } & \text { zeigern der vereinserrichtung aber vorher Gelegen- } \\ \text { heie Einwände zu erfahren und die Sta- }\end{array}$ darf (zur steuerlichen Behandlung von Vereinen tuten zu verbessern. ${ }^{21}$ Das Vereinsregister wird beim siehe Kapitel III/5).

Aus Sicht von österreichischen Rechtsexpertnnen und CSO-VertreterInnen ist es unproblematisch, Vereine zu gründen oder sich in einer anderen Rechtsform zusanmenzuschileßen (Interview 7, 12, I5 und I9). Es existiert ein durchaus bürokratischer, aber weder hinderlicher noch einschränkender Rechtsrahmen zur Gründung von Vereinen, Stiftungen und anderen Arten von Nonprofit- und Nichtregierungsorganisationen (Interview 7), die soziale, politische, kulturelle und sonstige gesellchaftliche Ziele verfolgen.

Die hăufigste Form der Vereinigung, die CSOs wählen, ist die Rechtsform des Vereins. Dies liegt daran, dass die Bedingungen fur die Grundung von eise macht genaue Vorschriften zu den St Dusen, den macht genaue Vorschiften zu den Statuten, den fugnissen Die Behörde erlaubtid. R. durch Fristabfauf oder ausdrückliche Einladung mit Bescheid innerhalb von längstens sechs Wochen Becheid langen der Vereinsstatuten die Aufnahme der Vereinstätigkeit. Nur bei dem Verdacht des Vorliegens der Voraussetzungen des Art. II Abs. 2 der EMRK zum Schutze der Menschenrechte und Grundfreiheiten ${ }^{20}$ kann die Vereinsbehörde innerhalb derselben Frist mit Bescheid erklären, dass die Gründung Bundesnormen\&\&Gesestzesnummer=200001977 abgerufen an

20 Nach Art. 11 Abs. 2 der EMRK darf die Ausübung des Grundrechts der Versammlungs- und Vereinigungstreiheit theinGesetz vorgesehenen, die in einer demokratischen Gesell-
schaft im Interesse der nationalen und offentlichen Sicheschaft im Interesse der nationalen und offentlichen Siche-
heit, der Aufrechterhaltung der Ordnung und der Verbrechensverhütung, des Schutzes der Gesundheit und der Mo oder des Schutzes der Rechte und Freiheiten anderer notwendig sindk Abfragen über die Website http: / / zvr.bmi.gv.at ö fentlich zugänglich.

Vergleichsweise unterentwickelt sind in Österteich gemeinnützige Stiftungen, was in den letzte Jahren zu vermehrten Forderungen nach einer Re(fitungsrechts gefuhrt hat. Derzeit sind sowohl privat- als auch gemeinnützige Stiftungen im Privatstiftungsgesetz (PSG) geregelt. ${ }^{22}$ Stiftungen dienen in erster Linie zur Verwaltung von Vermögen Bei Schaffung des Stiftungsrechts im Jahr I993 stand der Anreiz im Mittelpunkt, Kapital im Land zu 000 Privatiftungen in Österreich sind ren rund . 20ro). Ziel ist es, in eigenes Stiftungsecht fïr gemeinnïzige Stiftungen zu schafren um besseneize zu setzen, Kapital für gemeinnüzige Zwecke Verfügung zu stellen. Fine entsprechende Reform des Stiftungsrechts zur Förderung des gemeinnützigen Stiftungswesens ist im Regierung 2013-201 $8^{23}$ verankert.

Bei der Gründung von Vereinigungen oder juristischen Personen gibt es im Wesentlichen keine Bezug auf Alter, Geschlecht oder Nationalität (Interview 7, I2 und I9). Jüngste Beispiele in Österreich eigen, dass für die Gründung und das Führen von CSOs auch das 'Gesinnungsstrafrecht r relevan sein kann, nämlich dann, wenn diese unter Verdacht stehen, eine 'kriminelle Vereinigung $₫$ nach $\$ 278$ a Strafgesetzbuch (StGB) ${ }^{24}$ darzustellen (Interew 7 und I2).

Eine Registrierung von zivilgesellschaftlichen Vereinigungen ist in Österreich nicht zwingend e forderlich. Es gibt keine Verpflichtung für große Gruppen, wie etwa Kirchgangerimnen oder Fußballfans, sich als Verein zu registrieren. Allgemein is

21 h rößeren Schranken oder Diskriminierungen in eine Registrierung für Personen und Körperscha ten, die im kaufmannnischen und unternehmerschen Bereich tatig sind, vepsitend (s 8 U GB) Die Registich

http:// www.bmi.gv.at/cms/BMI_Vereinswesen/gruen-
dung/Anzeige_d_Errichtung.aspx abgerufen am 28.04.2014

22 https:// /www.ris.bka.gv.at/GeltendeFassung. wxe?Abfrage=Bu ndesnormen \&Gesetzesnummer
W=True abgerufuen am 28.04:2014

23 http:// www.bka.gv.at/DocView.axd?Cobld=53264 abgerufen am 05.03.2014. 24 https:// www.ris.bka.gvvat/Dokument. wxe?AAbrage=Bundesn
rmen\&Dokumentnummer =NOR40033827 abgerufen am

https:// www.ris.bka.gv.at/Dokument

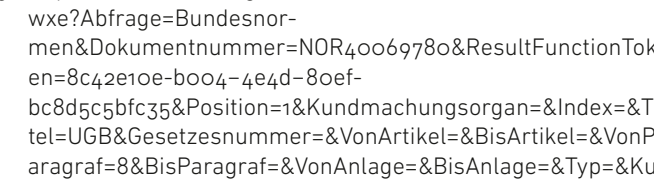
ndmachungsnummer $=\&$ Unterzeichnungssda aggerufen $a m$
nat
Vorteile. Dazu zählen Haftungsgründe, bestimmte steuerliche Vorteile im Zusammenhang mit der Gemeinnützigkeit und Spendenbegünstigung oder die inanzielle Unterstützung durch Förderungen von der öffentlichen Hand, die oft Anlass für die Registrierung eines Vereins, einer GmbH oder einer Privatstiftung sind. Während Vereine beim Vereinsregister anzuzeigen sind, werden juristische Personen, wie die GmbH, Privatstiftung oder die Genossenschaft, beim Firmenbuchgericht registriert. Die Registrierung von Vereinigungen bringt Vorteile aber auch Verpflichtungen mit sich. Bei Vereinen besteh die Verpflichtung zur Bestellung von Leitungs- und Kontrollorganen, zur regelmäßigen Abhaltung von Mitgliederversammlungen und zur Beachtung bestimmter Rechnungslegungsvorschriften. Dies gilt für alle registrierten vereinigungen, unabhängig von der jeweiligen Grổe, also fur kleine Vereine mi wenigen Mitgliedern bis hin zu großen Vereinen, wie dem österreichischen Gewerkschaftsbund oder dem Automobilkub OAMTC mit jeweils über einer Millon Miglieder (ntervew 7 ). Fur große Vereinander folgenden Rechnungsjahren jeweils höher als eine Million Euro waren, gelten ähnliche Rechnungslegungsvorschriften wie für Profit-Unternehmen (\$ 22 VerG).

Eine Besonderheit ergibt sich, wenn man Berechtigungen, z. B. Umweltverträglichkeitsprüfungen (UVP), als BürgerInneninitiative, wahrnehmen möchte (Interview 7 und I9) Bei besonders relevanten öffentlichen und privaten Projekten

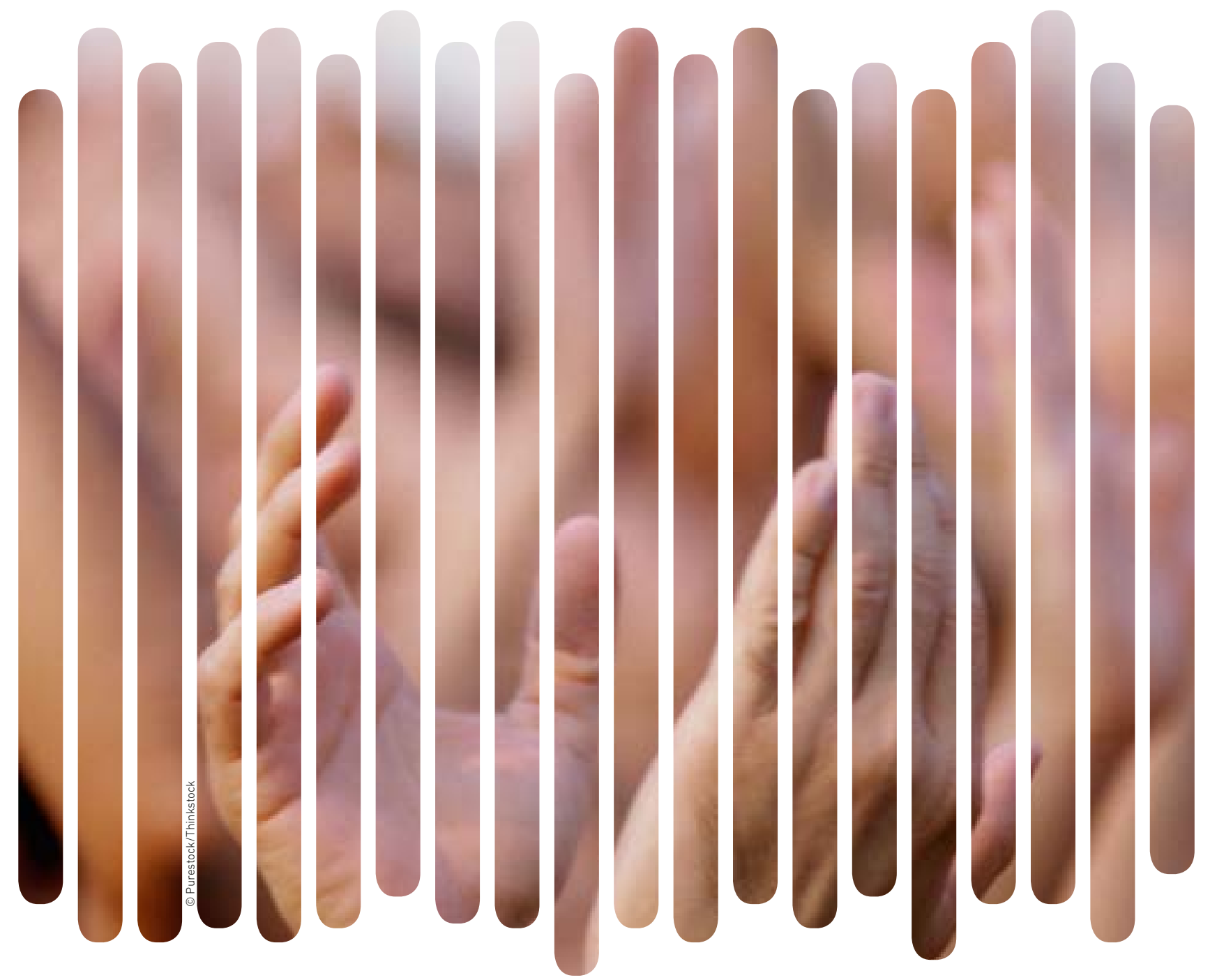

EU-Richtlinie ${ }^{26}$ sowie dem nationale esetz (UVP-Gesetz) die Umweltauswirkungen eietz ${ }^{27}$ ist ein Sonderfall, weil in diesem Verfahren einer BürgerInneninitiative ein eigener Rechtschameindestens 200 Personen, die in der Standort(ines Projektes oder einer unnittelbar (Gemerien wor gernnneninitiative Partei- bzw. Beteiligtenstellun erlangt, ist eine Stellungnahme samt Unterschrif作 Une (a) Gifen ul ond zu machen, Rechtnittel zu er-

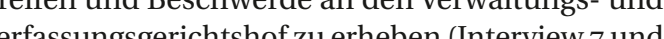

http:// eur-lex.europa.eu/LexUriserv/LexUriserv.do?uri=0J 2012.026.0001::0021:DE:PDF abgerufen am 28. 04.2014

hittps:// www.ris.bka.gvvat/GeltendeFassung. wxe?Abfrage=-
BundesnormenzGesetzesnummer=10010767 abgerufen am 28.04.2014

http:// www.umweltbundesamt.at/umweltsituation/uvpsup /

/verfahrensablauf/beteiligung abgerufen am es Vorhabens, wie etwa für den Bau einer Autorkter zugeschrieben wird, so dass ein Bevollmächgter für die ganze Gruppe handeln kann (Interew 19). Eine BürgerInneninitiative ist eine Gruppe

05.05 .2014 

regierungsorganisationen gründen.

2. Natürliche und juristische Personen werden im Falle einer Nicht-Registrierung ihrer Organisationen nicht sanktioniert benen Fristen wirklich moglich, Behorden entscheiden über Anträge in einer nicht subjektiven und unpolitischen Weise. innerhalb und außerhalb ihrer Heimatländer gründen bzw. sich an innen beteiligen

Die interviewten Rechts- und CSO-VertreterInne konnten keine Beispiele dafür nennen, wo die Grü dung von CSOs untersagt oder eine Nicht-Registrierung sanktioniert wurde. Einzelpersonen und CSOs außerhalb von Österreich gründen bzw. sich an ihnen außerhalb vo
beteiligen.

Problematisch wird jedoch die Anwendun (

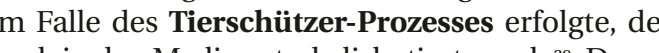
hobeneVorwurfnach $\$ 278 \mathrm{a} \mathrm{StGB}^{30}$ betrifft das Bilden einer kriminellen Vereinigung. Im Tierschützer-Prozess in Wiener Neustadt mussten sich im März 2010 I3 Tierschützer I4 Monate lang wegen Beteiligung an einer kriminellen Organisation vor Gericht verant-
worten. Für acht Angeklagte erfolgte im Mai 201 I der mittlerweile rechtskräftige Freispruch bezüglich des Vorwurfes der Bildung einer kriminellen Organis tion, die übrigen fünf standen bis vor kurzem weiterhin wegen des Vorwurfs der Nötigung, Sachbeschädigung, Widerstand gegen die Staatsgewalt und Tie qualerei vor Gericht. ${ }^{3{ }^{1}}$ Nach Ansicht der interviewten Rechtsexperten ist die Tierschutzbewegung in Oster作 iuvilgesellschaftliches Engagement zu kriminalisieen (Interview 7 und I2).

Es besteht die Gefahr dass diese Besonderheit auch für andere CSOs ausgelegt werden könnte. Nach Meinung der Interviewpartner (Interview 7, 12 15, 18, I9) kann mithilfe des Strafrechts gegenAnders denkende Osterreichenh lnstrumentarium, wenndasin Gang gesetzt wid, kann die Zivilgeselschant praktisch . CetzgebungnichtnurgegenCSOs sonderngundätrlich gegen die gesamte Gesllschaft, wonach solche Instrumente gen missliebige Personen oder Bevökerungsgruppen und abweichendes Verhate ingesetzt werden könnten (Interview 7 und I2).

Weitere Besonderheiten in der Praxis ergeben sich einerseits aus der Abhängigkeit mancher CSO vom Staat und anderseits aus der fehlenden Mitgestaltungsmöglichkeit von CSOs bei Entscheidungsprozessen der öffentlichen Hand. Die Abhängigkeit besteht aufgrund der Größe der Organisationen, de Finanzierung oder politisch involvierten Parteienstrukturen (Interview I2). Die fehlende (zeitgerechte)

Pro. $/ /$ derstandard.at $/ 1397521831000$ /Tierschuetzer-
Prozss-Neuauflage-startet-am-13-Mai abgeruffen a

http://www.parlament.gv.at/PAKT/VHG/XXIV/III/II__00348/ imfname_263117.pdf abgerufen am 05.03.2014 31 http://tierschutzprozess.at abgerufen am 05.03.20
Einbeziehung von CSOs in politische Entscheidungszesse wurde ebenso angesprochen (Interview I9). In einem konkreten Beispiel für Mitgestaltung wurde der Umweltbereich erwähnt. Österreich hat beteiligung unterzeichnet.Dadurch soll Transparen mit Zugang zu Informationen, Partizipation mit Zugang zu Genehmigungsverfahren und Rechtsschutz in Umweltverfahren gewährleistet werden. Nach diesemAbkonmenten Umweltschuzzorganisationen Beschwerde- und Klagerechte in allen umweltrelevanten Verfahren. Dieses Abkommen sieht vor, das zumindest die Umweltorganisationen das Recht haben, gegen Unterlassungen der öffentlichen Hand in Umweltbereich gerichtlich vorzugehen. Das Gerich verpflichtetdanndie Behörde tätigzu werden. DieAar(a)

\section{VERBESSERUNGSPOTENZIALE}

Im Wesentlichen wird das Recht auf Vereinigungsfreiheitoderdie MöglichkeitsichzusammenzuschlieBen in den Interviews als unproblematisch eingete Kritik betrifft die 1rarechtlichen Konsequendu bilden (u a $\$$ z 7 a StGB). Daher wird an die der Gesetzgebung Gesinnungs und nicht nurWorte zu ändern (Interview 7 und Das ist etwas, was durch einfoche Worte nicht geändert oder verbessert werden kann (Interview 7 ). Ein weiteres Verbesserungspotenzial aus rech icher Sicht ist, das Vereinsregister nicht beim Bundesministerium für Inneres zu führen, sondern - in Anlehnung an das Firmenbuch - bei den Gerichten. Aus rechtlicher Sicht sind Vereine Körperschaften. Nach Meinung eines Rechtsvertreters können Gerichte das Register durchaus transparenter führen und es ware darüber hinaus ein positiverer Zugan zur Vereinigungsfreiheit möglich. Dennach könnte dies als eine Bewegung,$[\ldots]$ von der Polizei und der Kriminalität hin zur zivilgesellschaftlichen Verein gung $[\ldots]$ < interpretiert werden (Interview 7 )

Eine stärkere Einbeziehung von CSOs in politische Entscheidungsprozesse könnte über das Einführen von )Grünbüchern` (Diskussionspapiere zun wis bestich

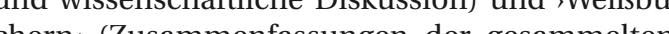
(2) lem angels̈̈chischen Paum biekar Prozess ist aus auch bei der det Dieser Wunsch nach Finbeziehung vo Bet-iligten zielt auf transparentere Entscheidungsprozesse der öfe trichen Hand ab. CSOs k̈̈nten sich dafür auf organisationaler Ebene mit anderen CSO verbünden und Allianzen bilden. Im Konkreten wurde die Umsetzung der Aarhus UN-Konvention für die Mitgestaltung von CSOs im Umweltbereich gefordert (Interview I9).

An die Adresse der CSOs richtet sich der Vo schlag offen ubber bestehende Abhängigkeiten zwschen CSOs und öffentlicher Hand zu reflektieren. Starke Dachverbände und gemeinsame Interessensvertretungen von CSOs, die gegen inhaltliche furr besonders wichtig (Interview I 2 )

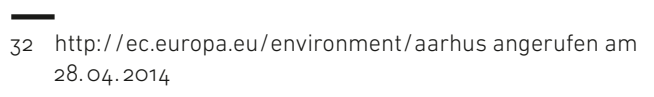

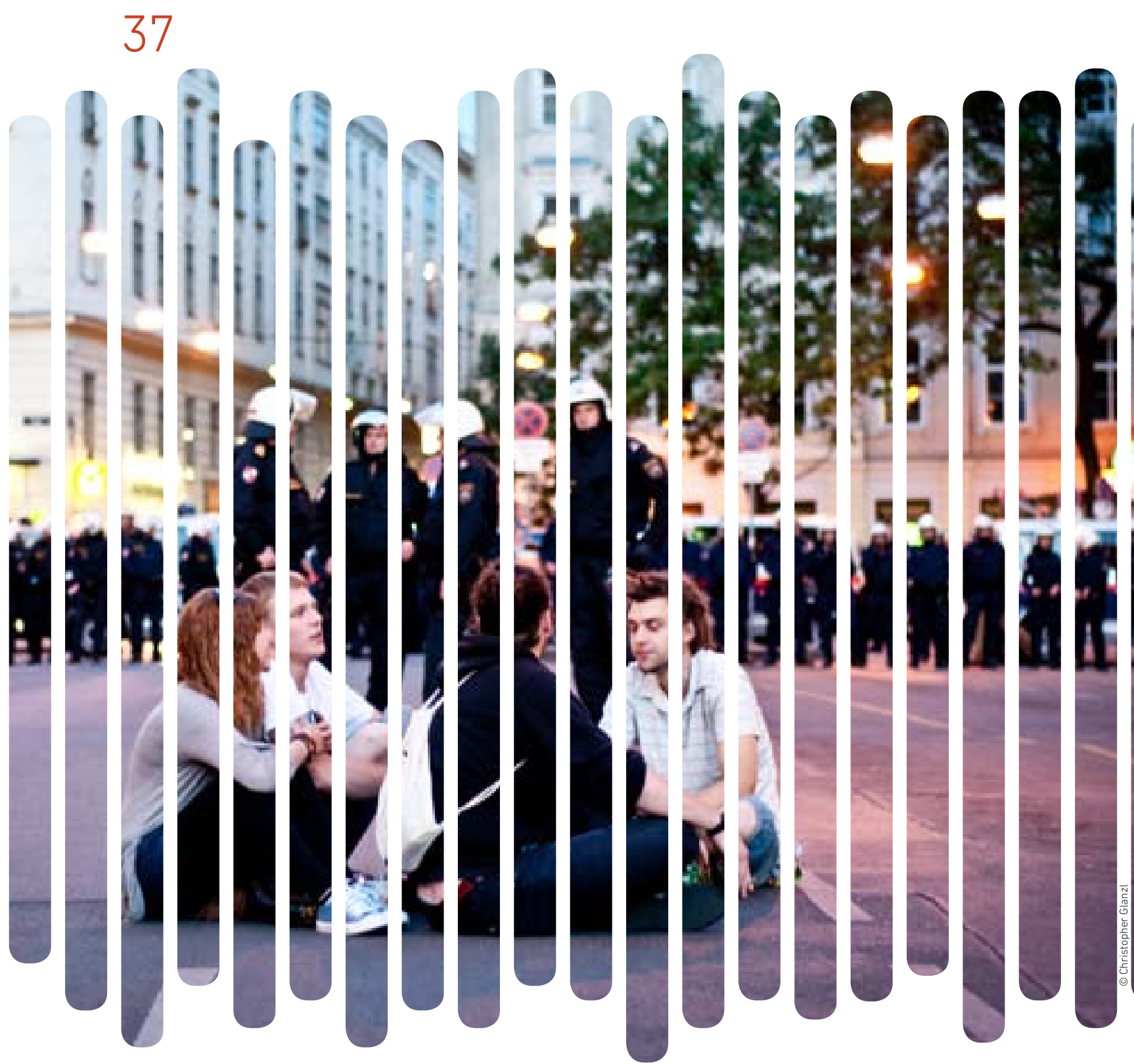

3. Versammlungsfreiheit

ie Versammlungsfreiheit in Österreich ist verfa sungsrechtlich in Artikel I2 Staatsgrundgese 2 (StGG) sowie Artikel II Europaische Mensche (E) Versammlungsgesetz (VersG) ${ }^{34}$ geregelt. Sie berech-
tigt Personen, sich zur Verfolgung gemeinsamer Anlige Penson, sich zurVefol gung geneinsaner AnDegen zu versamme zond Cegensatz zur Vereinigungsfreiheit ist die VerGemment schränkt Versammlungen sind vorïbergehende, nicht zufällige Vereinigungen mehrerer Personen an einem bestimmten Ort. Das Versammlungsgesetz selbst enthält keine Definition des Begriffes Versammlung, es setzt diesen vielmehr voraus. Nach

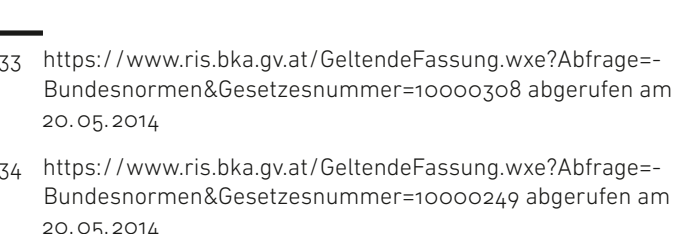

ândiger Judikatur des Verfassungsgerichtshofes ${ }^{3}$ ist eine Zusann als wes anzulet (Diss e kommenen entsteht.

Nach dem Versammlungsgesetz (VersG) sind Volksversammlungen und allgemein zugänglischnitt wird der Frage nachgegangen, wese Abconderheiten in Beze aufdieVeron, was die Bein Österreich sind. Erwähnenswert ist auch die Bannmeile. Während der Nationalrat, der Bundesrat, die Bundesversammlung oder in Landtag versammelt ist, darf im Umkreis von $300 m$ von ihrem Sitz keine Versammlung unter freiem Himmel stattfinden ( $\$ 4$ und 5 VersG).

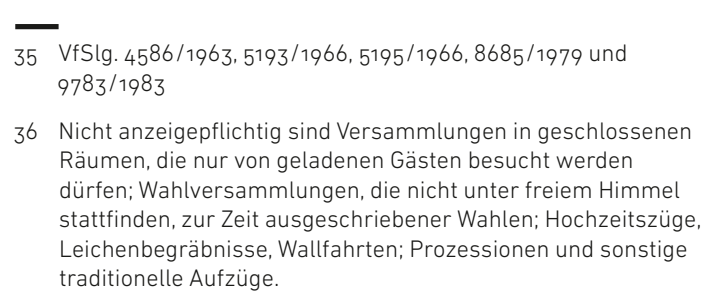




\section{RECHTLICHE REGELUNGEN}

\section{RECHTLICHE REGELUNGEN-INDIKATOREN}

Denäche Rahmen basiert auf internaliona frand alle ohne Diskriminierung.

2. Die Gesetze kennen spontane, simultane und Gegenver-

3. Die Ausübung des Rechts unterliegt nicht der Genehmigung durch die Behörden, sondern höchstens einer vorherigen Anzeige, die nicht schwierig ist.

4. Alle gesetzlichen bzw. durch die Behörden vorgeschriebenen Einschränkungen des Rechts können von den Organisatorlnnen angefochten werden.

Das Grundrecht aufVersammlungsfreiheit basie auf internationalen Standards gemäß Europäscher Menschenrechtskonvention (Art II Absatz I EMRK). Die Ausübung der Versammlungsfreiheit wurde in langwierigen gesellschaftlichen Auseinandem Verfassungsrang ausgestattet und das Versammlungsgesetz (VersG) von 1953 ist im WesentlMale bis heute gültig und wurde lediglich einige Ausübung dieses Menschenrechts und wird von RechtsvertreterInnen und CSO-VertreterInnen als view 7 , I2 I5 nichd

\section{Einschränkungen sehen die Gesetze jedoch} für Menschen ohne österreichische Staatsbürge schaft vor. Laut Versammlungsgesetz ist die Anzeige, Leitung und Ordnung von Kundgebungen und Demonstrationen ausschließlich österreichischen StaatsbürgerInnen vorbehalten. Auch in Art I2 StGG wird lediglich das Recht österreichischer Staatsbürungsgesetz (\$\$ 8VersG) heißt es, dass Ausländerlnnen weder als VeranstalterInnen noch als OrdnerInnen oder LeiterInnen einer Versammlung zur Verhandlung öffentlicher Angelegenheiten auftreten dürfen, an einer Teilnahme werden sie durch das Gesetz nicht gehindert. ${ }^{37}$ Hier steht das Versammlungsgesetzin Widerspruch zur Judikatur des Europäischen 16 der EMRK, gemäß der jedenfalls auch EU-AusländerInnen das Recht haben sich friedlich zu versammeln. Weiters widerspricht die Beschränkung

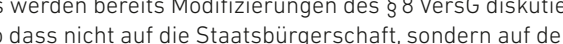
so dass nicht aut die Staatsburgerschaft, sondern auf den
Aufenthaltsstatus abgestellt wird (Interview 7). Für Persone ohne ordentlichen Aufenthaltstitel ist in der Praxis auch die
Teilinahme an Demonstrationen und Kundgebungen roble-

matisch, da das auf Staatsbürger Art 2I des Internationalen Paktes über bürgerliche und politische Rechte (IPBPR) ${ }^{38} \mathrm{de}$ UNO, der allen Menschen das Recht auf Versammlungsfreiheit zuerkennt. Anlässlich der Ratifizierung des Paktes hat Österreich allerdings dazu einen Vo behalt erklärt, sodass Österreich nicht vertragsbrüchig ist. Eine explizite Anpassung des Versammlungsgesetzes an die Erfordernisse der EMRK wäre notwendig um in dieser Frage Klarheit zu schaffen. Darüber hinausgehend sollte der Vorbehalt zum IPBPR zurückgezogen und das Recht auf Versamm-

Die Gesetze kennen die Begriffe der sponta-

enenversammlungen nicht. Nach Meinung von Rechtsvertren und CSO-VertreterInnen sind diese Forse on (3) ich dourch aus, dass sie nicht angez zeichnen während unter simultanenVersammlungen zeitig stattfindende Veranstaltungen zu verser s̈rren oder zu sprengen, dann kann dies als StrafVersanten

Versammlungen sind spätestens 24 Stunde vor ihrem Beginn bei der Behörde anzuzeigen. Sie bersamm tionen als genehmigt, solange sie nicht untersagt sind ( $\$ 6$ VersG). Die VeranstalterInnen sollen lediglich nachweisen können, dass sie den Sicherheitsbehörden die Mitteilung gemacht haben. Bei eine Zusammenkunft von mehr als drei Personen, die ein gemeinsames politisches Wirken verfolgen, sind Privatpersonen oder CSO-VertreterInnen verpflichtet, Anzeigen bei der Bundes-, Landespolizeidirektion oder Bezirksverwaltungsbehôrde vorzunehmen (\$2 Absatz I VersG). Allerdings dürfen ersammlungen nicht automatisch untersagt bzw. aufgelöst werden, sofern sie friedlich sind (Art II Absatz 2 EMRK; Interview 7 und I2) Liegt keiner der im Art I I Abs 2 EMRK angeführten Umstände vor, kann eine Spontanversammlung nicht untersagt (bzw aufgelöst) werden (\$\$ I3, I4 VersG). Die untellassene Anzeige der Versammlung stellt jedoch eine Verwaltungsübertretung des ad hoc-Veranstalters dar ( $\$ \$ 2$, I9 VersG), wenn nich is Eunz

38 hitp: // www.ohchr.org/EN/Prof
aspx abgerufen am 20.05.2014

Das Versammlungsgesetz (VersG) unterscheidet in 0sterreic nur Zwischen den Begriften angezezigtes bzw. nicht ange-

Bis zu 1 Jahr Strafdrohung bei Sprengung einer Versammlu (\$284 StGB), bis zu 6 Monate Strafandrohung bei Ver
rung oder Störung einer Versammlung (\$ 285 StGB).

Eine Untersagung oder Auflösung einer Versammlung durch die Behörde darf nur stattfinden, wenn dies aus einem der in Art 11 Absatz 2 EMRK genannten Gründe stattfindet. Dies kann
sein: im Interesse der nationalen und der ôffentlichen Sicherchensverhüutung, des Schutzes der Gesundheit und der Moral oder des Schutzes der Rechte und Freiheiten anderer.
Die VersammlungsanzeigerInnen und Ver- oder Verstöße gegen das im Versammlungsgesetz sammlungsleiterInnen müssen nicht dieselben Per- verankerte Vermummungsverbot vorliegen (\$9 sonen sein. Die Anzeige kann als Fax geschickt wer- VersG) sind die Behörden berechtigt Versammlun den, wobei die voraussichtliche Anzahl der Teilneh- gen sofort aufzulösen ( $\$$ I3 VersG), werden jedoch merlnnen, der Zeitraum, die Route bei Demonstra- angehalten verhältnismäßig vorzugehen und die tionen und die voraussichtlich verwendeten Mittel, maßhalten terview I5, I7 und I8).

Eine im Vorfeld der geplanten Versammlung

Alle Einschränkungen des Rechts können von den Behörden kommunizierte Untersagung von den Organisatorinnen angefochten werden, kann von den VeranstalterInnen bei den Behörden jedoch werden Berufungen kaum genutzt. , Ver- angefochten werden, was jedoch mit Kostenrisiken sammlungen, deren Zweck den Strafgesetzen zuwi- verbunden ist (Verfahrenskosten) und in der Praxis derläuft oder deren Abhaltung die offentliche Sicher- aufgrund langerVerfahrensdauer irrelevant ist und heit oder das offentliche Wohl gefahrdet, sind von der daher von zivilgesellschaftlichen Initiativen kaum Behörde zu untersagen (\$ 6 VersG). Wenn Gesetze genutzt wird (siehe auch unter Besonderheiten in direkt vor Ort der Versammlung verletzt werden der Praxis).

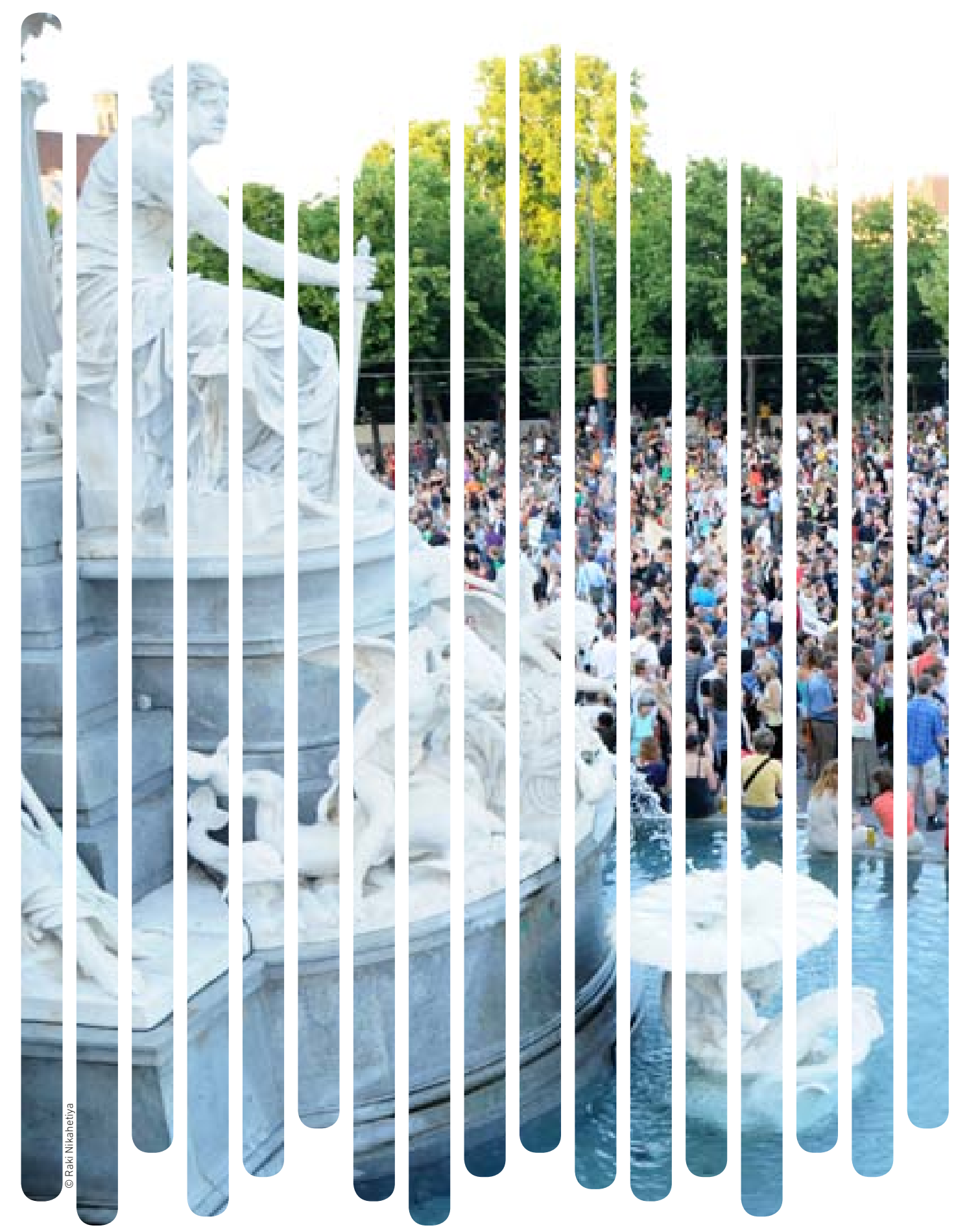


Bei Kundgebungen und Demonstrationen, mittag für Versammlungen am Sonntag problemadie auf Privateigentum erfolgen, besteht die Gefah

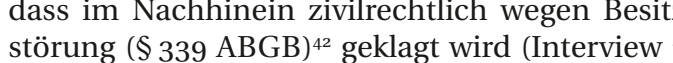
I8). Es können außerdem Schadensersatzforderungen entstehen, z. B. wenn Eigentum beschädigt
wird (z. B. Rasen) oder wenn Unternehmen in ihrer Erwerbsfreiheit beschränkt worden sind (Interview I8).

\section{BESONDERHEITEN IN DER PRAXIS}

\section{iil) PRAXIS-INDIKATORE}

Ss sind keine Falle von Eingriffen in die Versammlungsfreiheit aufgetreten und jede Gruppe von Menschen kann sich im Einklang mit den gesetzlichen Bestimmungen an

gewunschten Orten und Zeiten zusammenfinden.

. jede Einschränkung der Versammlung unverzüglich und schriftlich an den/die VeranstalterIn übermittelt, um die Möglichkeit der Berufung zu garantiere

3. Gleichzeitige, spontane und Gegenversammlungen können stattfinden und der Staat erleichtert und schützt Gruppen, Rer sie daran hindern Oder storen möchten.

Einschränkungen des Rechts können von vorgeschrieben Einschrankungen des Rechts
rlnnen angefochten werden.

5. Es wird keine unverhältnismäßige Anwendung von Gewalt durch die Vollzugsbehörden ausgeübt, inklusive präventive Organisatorlnnen und Teilnehmerlnnen. b. Die Medien haben uneingeschränkten Zugang auf Versammlungen.

Die politische Kultur für die Ausübung des Menschrechts auf Versammlungsfreiheit hat sich in Öste reich über viele Jahre entwickelt. In den meisten Fällen können sich zivilgesellschaftliche Initiativen ohne Einschrănkungen versammeln. Vor allem etablierte und bekannte CSOs sind in der Regel mit keinen Schwierigkeiten in der Umsetzung von Demonstrationen und/oder Kundgebungen konfrontiert. Problematischer ist die Situation für kleine Initiativen oder lose Bündnisse, für die Haftungsrisiken existenzbedrohend werden können. Daher werden Versammlungen in vielen Fällen von größeren Organisationen wie der Osterreichischen HochschülerInnenschaft $(\mathrm{OH})$, politischen Parteien oder dem Österreichischen Gewerkschaftsbund ange-

Da Versammlungen in Österreich nicht bewilligungspflichtig sind, sondern nur einer NichtunBehörde die Anzeige der Versmmlung in ner Nichtuntersegung aufVerlangeng imriftlich bestätigt (\$ 2 Absatz 2 VersG). Die Öffnungszeiten der für Versammlungsanzeigen zuständigen Landespolizeidirektion bzw. die Bezirksverwaltungsbehörde (Bezirkshauptmannschaft oder Magistrat), führen dazu, dass in der Praxis die vorgeschriebene 24 Stunden-Frist der Anzeige z. B. am Freitagnach$\overline{42}$

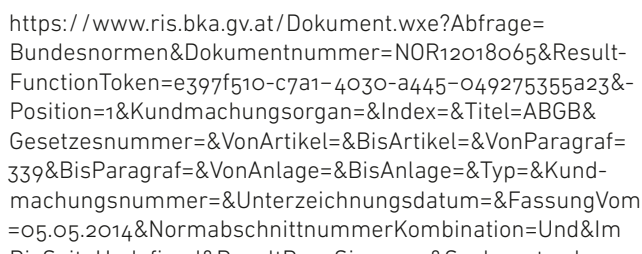

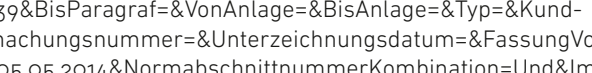

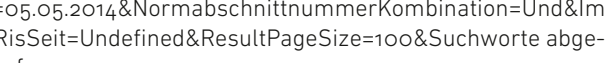

\section{Die Sicherheitsbehörden gerieten in den letz-} ten Jahren des Ofteren wegen der Untersagung von Kundgebungen in die Kritik von CSO-VertreterInnen. Als Beispiele können hier angeführt werden Kein Europa des Rechtsextremismus (24. 0I. 20I4 Jetzt Zeichen Setzen)'3, LKW-Blockade Grenzübergang Grametten (3I. I0. 2013, Verein Neue Thayatalbahn) ${ }^{44}$, Rasen am Ring (IG Fahrrad 22. 09. 20II), Wiener Akademikerball (WKR)-BalDemo (28. 0I. 20I I, Antinationales Bündnis Wien).

In der Causa Rasen am Ring wurde von der IG Fahrrad eine Beschwerde gegen den Bescheid de Bundesministerin fur Inneres wegen Einschränkun der Versammlungsfreiheit angestrengt. Das E kenntnis des Verfassungsgerichtshofs (04. 03. 2014) kritisiert, dass die Behörde die zu erwartenden sschwerwiegenden Behinderungen als Grund für das Verbot habe genügen lassen, obwohl es den Veranstalterlnnen genau darum gegangen sei, öffentlizen Der Bund muste den Bescheid auf zu benütdie Prozesskosten in der Höhe von 2856 Euro zhlen (VfGH 04.03 . leitete rechtliche Schritte gegen die Untersagung WKR-Ball-Demo 2013 wurde vom VfGH 201 Recht erkannt 47 . dass lein der Umstand eines Risikos von Auseinandersetzungen nicht rechtfertigt eine geplante Versammlung zu untersagen. Fin vorbeugendes Versammlungsverbot ist nicht zulässig. Der angefochtene Be scheid wurde aufgehoben und der Bund wurde verpflichtet 2.400 Euro Prozesskosten zu übernehmen. Auch von den InterviewpartnerInnen wurden Repressionen durch Platzverbote und Untersagunn erwähnt (Interview 7, I 7 und I9).

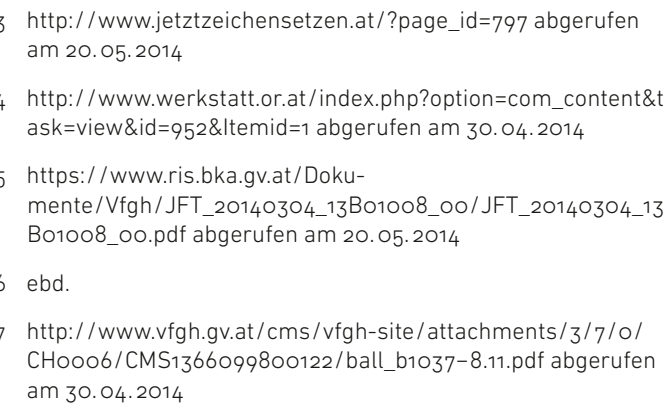




\section{FUU DIEIVILGESELLISCHANT} lung kann rechtliche Konsequenzen bedeuten. für die Wahrung der friedlichen Versammlun durch Vorkehrungen, wie OrdnerInnen, zu sorgen (S II VersG). AnzeigerInnen von Versammlungen, können bei eventuellen Schwierigkeiten rechtlich zur Rechenschaft gezogen werden. Haftungsrisike werden dabei als besonders problematisch betrac tet (Interview I8). Sie können außerdem wie TeilnehmerInnen von harten Strafandrohungen in Zusammenhang mit dem Vorwurf des 'Landfriedensbruch (\$ 274 StGB) betroffen sein. Aktuell zum I. Mai 2014 ermittelt die Staatsanwaltschaf auf Basis des Landfriedensbruchsparagraphen gegen rrund 500 noch unbekannte Personen«. Das bedeutet, dass friedliche DemonstrationsteilnehmerInnen allein aufgrund ihrer Teilnahme san einer Zusammenrottung einer Menschenmenge, die darauf abzielt, daß unter ihrem Einfluß ein Mord ( $\$ 75)$ ein Totschlag (\$ 76), eine Korperverletzung (\$\$ 83 bis 87) oder eine schwere Sachbeschadigung (\$ I26) önnen, wennes zu einer solchen Geultat gekonmen ist.$^{53}$

2002 wurde unter der ÖVP-FPö Regierung da (

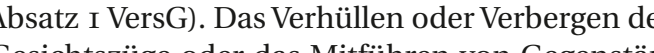
den die ihrem Wesen nach dazu bestimmt sind, die Feststellung der Identität zu verhindern (VersG $\$$ S können demnach mit Freiheitsstrafen bis zu sech Wochen und Geldstrafen belangt werden. Dieses Vermummungsverbot wurde medial auch im Zuge des Akademikerballs 2014 diskutiert, da dieses Ve bot des Verhüllens im Winter, etwa durch Mützen und Schals, ausgeweitet auf neun Wiener Bezirke as úberzogen beurteilt wurde. ${ }^{54}$ Die oben genan Strafandrohungen dienen nach Meinung der CSO-VertreterInnen auch dazu, Personen massiv einzuschüchtern (Interview I7 und I8).

In Osterreich werden nach Anzeige Untersagungen mit Erlâuterungen eines Grundes den VeranstalterInnen übermittelt. Wie oben dargestellt, gibt das osterreichische Recht VeranstalterInnen die Möglichkeit gegen Untersagungen Beschwerde einzulegen. Die meisten machen davon jedoch aufgrund des hohen Aufwands und einer Verfahrensdauer von meist mehr als zwei Jahren nicht Gebench ien is Ber Anlus erstrichen und die Aufhebung damit für die Veran talternnen in der Praxis irrelevant (Interview 7,15 und 1 ) Die Mörlichkit ineverten fung ist demnach praktisch nicht gegeben. Die Versammlung kann nur an einen anderen Ort ner anderen Zeit und auf andere Veranstaltungsform ausweichen (Interview 7 und I8). In Deutschland gibt es die Möglichkeit, Eilverfahren be

$\overline{53}$

http:///albertsteinhauser.at $/ 2014 / 03 / 25 / \mathrm{mi}$
landfriedenstruch rech abgerufen

54 http://www.wienerzzitung. at//nachrichten/wien/stadtpoabgerufen am 30.04.2014

55 Die Teilnahme an nicht angemeldeten Kunddebungen kann
von den Behörden mite einer Verwaltungsstrafe (\$ 19 V VersG)
In Osterreich werden Gruppen, die ihr Recht Versamm lungsfreiheit ausüben, durch die Behörde gegen Menschen, die sie daran hindern oder stören möchten, in der Praxis geschützt (Interview . Gleichzeitige, spontane Gegenversammlungen die nicht rechtswidrig sind, können stattfinden. Erwahnenswerte Versammlungen und Gegenver-

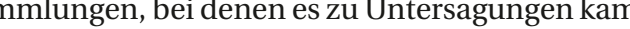
tetzter Zeit in Osterreich in Zusammen(ruluer WKR-Ball) der Wiener Hofburg statt. 20I4 hat es dazu dre angezeigte Demonstrationen gegeben. Von el(n) dass die Polizei emprohlen hat, ein Gedenken von (M (Meria-Platz) abzuhalten (

in Ö gationen . Fahrü in wegs sind Bei selchen Versamient die Polizei spontan den Verkehr aufzuhalten und umeren von der Polizei aufgelöst werden. Strafen sind oft wegen der Verwaltungsübertretung zu bezahlen, wenn TeilnehmerInnen die Kundgebung nicht verlassen. (Interview I7). Modern geworden sind auch Fash-Mobs als nicht angemeldete Versammlungen,

Ibvaoo2801.html oder http:/ / www.bverfg.de/ entschei-

30.04.20

http:// derstandard.at/13898579886021/Akademikerbal--
Kundgebung-abgesagt abgerufen am 30.04.2014

\section{3}

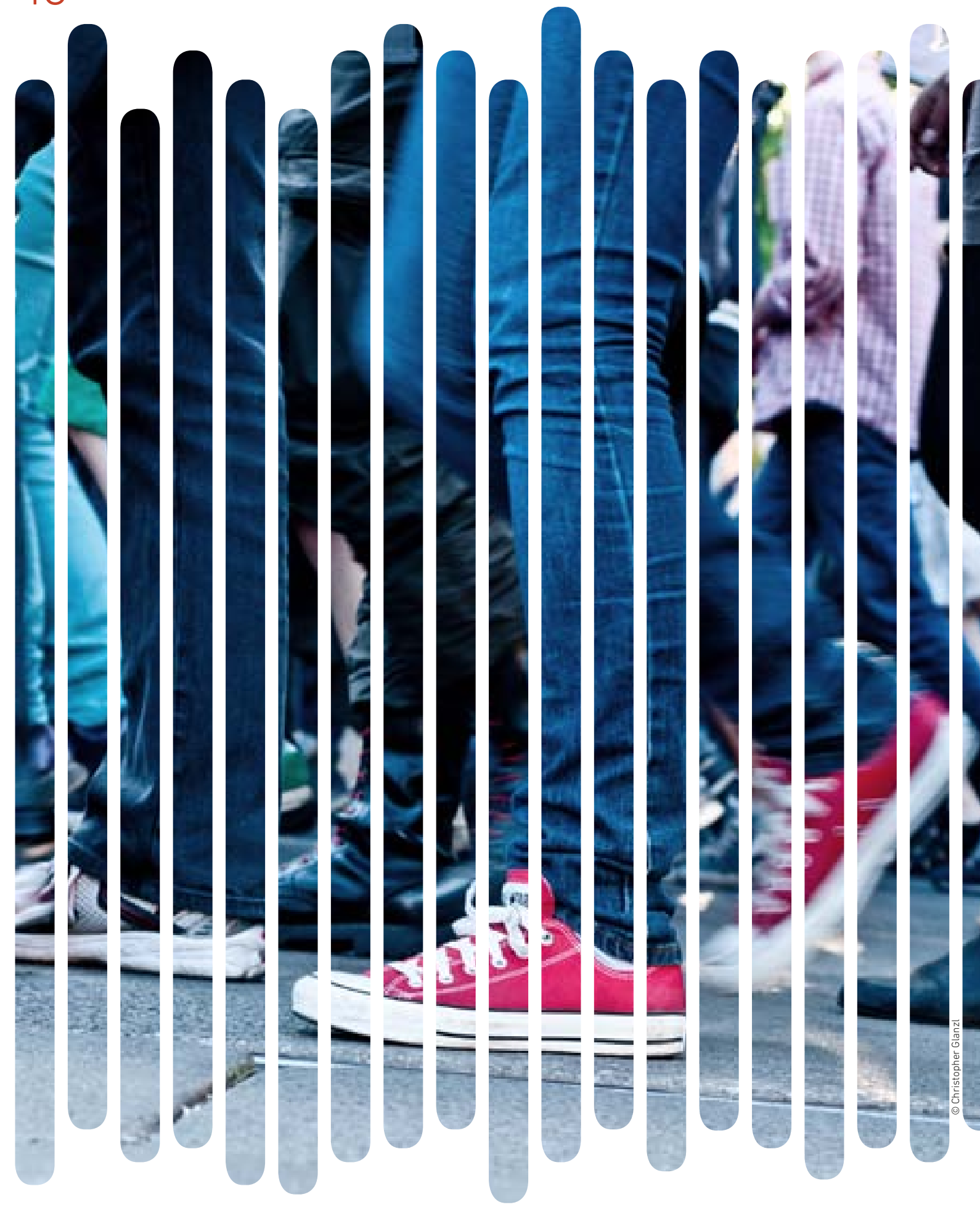

bei welchen weniger politische Anliegen verfolgt ihr Recht auf Versammlungsfreiheit ausüben und werden. Da solche Versammlungen sehr schnell werden von den Behörden darin geschützt. Von den wieder vorbeisind, greift die Polizeikaum in (Inter- Inteviewpartnernnen wurde jedoch Kritkam Ein view I5 und I7). Der Schutz der Polizei ist bei ange- satz von Gewalt in einigen Fällen durch die Vollzeigten Veranstaltungen ein Vorteil, wenn z B. der zugsbehörden geäußert (Interview I2, I5. I7 und Verkehrsstrom röräumisus Von den InterviewpartnerInnen wurde kein und Kontrolle von VersammlungsteilnehmerInnen konsistentes Vorgehen bei der Nicht-Untersagung wird in der Literatur als ,Policing of protest be von Gegenversammlungen wahrgenommen. Diese zeichnet. Nach dem Grundsatz der VerhältnismäInkonsistenz kann unverhältnismäßig sein, wenn Bigkeit ( $\$ 29$ Sicherheitspolizeigesetz) soll mögliVeranstaltungen längerfristig geplant und bewor- chen Straftaten präventiv vorgebeugt bzw. sollen ben werden und dann kurzfristig aus für Veranstal- begangene Straftaten geahndet werden..$^{57}$ erInnen nicht nachvollziehbaren Gründen untesagt werden (Interview 7, I5, I7 und 18). Gewalt durch die Polizei gegen Versammlungsteilnehm Sehr oft können österreichische StaatsbürgerInnen

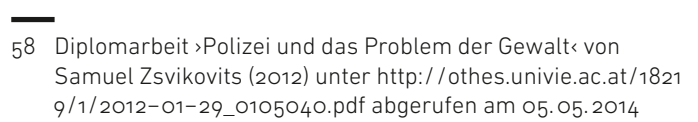


Einsatz von Gewalt gegen DemonstrantInnen war in den letzten Jahren insbesondere jährlichen Demonstrationen gesondere bei den kerball (früher WKR-Ball) oder bei den Demonstrationen gegen das ,Heldengedenken ‘ der deutschnationen gegen das 'Heldengedenken der deutschnationalen Burschenschaft Olympia, bei den gen Jahren den Opernball-Demos zu beobachten. Versammlungsteilnehmende sind - wenn es zum Einsatz von Gewalt kommt - mit dem Einsatz von Schlagstöcken und Pfefferspraydosen, Fixieren von DemonstrationsteilnehmerInnen am Boden Einkesselungen oder dem Einsatz von Wasserwerfern konfrontiert. ${ }^{59}$ Von Seiten der CSO-VertreterInnen wurde außerdem von überzogenem Verhalten der Polizei durch Personenkontrollen oder Verhaftungen gesprochen (Interview I5, I7 und I8).

Die Wahrscheinlichkeit für den Einsatz von Gewalt gegen VersammlungsteilnehmerInnen ist dann besonders hoch, wenn die Polizei in den Reihen der DemonstrantInnen gewaltbereite TeilnehmerInnen vermutet. Diese Vermutungen können falsch sein und ein uberzoger isch wid betrachtet, dass dodurch in vien Fïlen auch fredliche Teilehm dist betroffen in den letzten Johren $\mathrm{Ma}$ Rnhmen zum Schutz der Menschenrechte. I 999 wurde der Menschenrechtsbeirat eingerichtet. Ende 2009 wurde das Programm POLIZ POLIZEI.MACHT.MENSCHENRECHTE ${ }^{60}$ gestartet, das schen und handlungsspezifischen Rahmen stärker in der Ausbildung von PolizistInnen verankert:

Es wird empfohlen in der Kommunikation vor und während Kundgebungen auf Gewaltandrohungen $\mathrm{zu}$ verzichten, um bei den Sicherheitsbehörden nicht den Eindruck der Gefährdung der öffentlichen Sicherheit zu provozieren. (Interview 7).

Es ist im Fall von unverhältnismäßiger Gewaltanwendung oder anderen unverhältnismäßigen Maßnahmen durch die Polizei, davon betroffenen VersammlungsteilnehmerInnen möglich, Maßnahmenbeschwerden (Art I30 Absatz I Ziffer 2 Bundesverfassungsgesetz sowie $\$ 88$ Sicherheitspolizeigesetz) zu erheben. Diese Möglichkeit ist jedoch mit relativ hohen finanziellen Risiken ver-

-

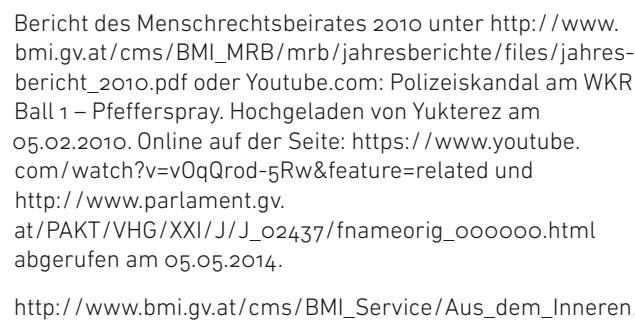

http://www.bmi.gvvat/cms/BMI_Service/Aus_dem_Inneren/
Die_Polizei_als_Menschenrechtsschutzorganisation.pdf abgerufen am 05.05.2014

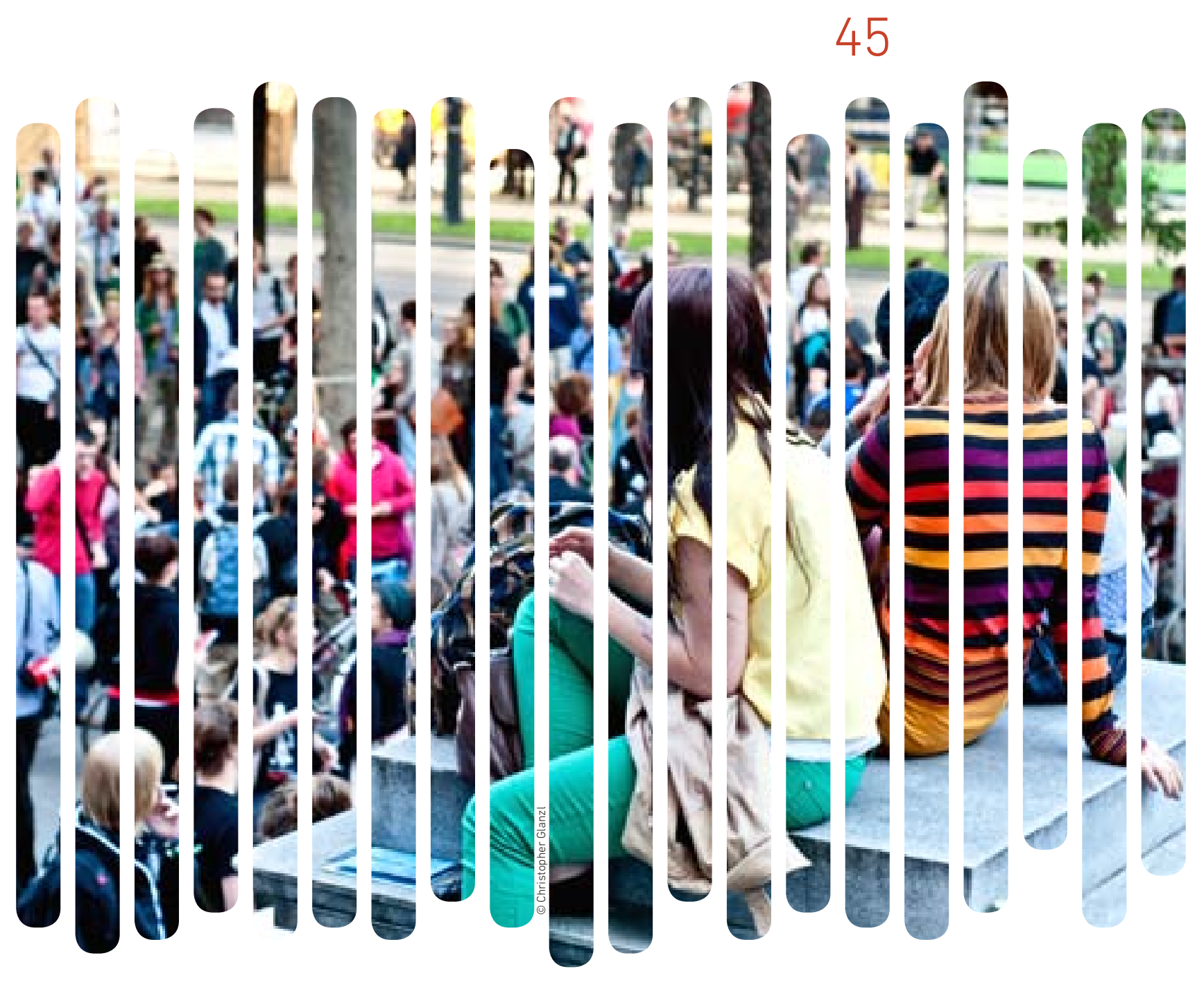

möglich. ${ }^{63}$ Dies traf kleine zivilgesellschaftlich organisierte Medien (z. B.: freie Radios) härter als große Medienunternehmen, wie den ORF und die Neue Kronen Zeitung, weil sie von den Behörden nicht so privilegiert behandelt wurden (Interview 7 und I8). Journalisten Club in einer Presseaussendung die massiven Angriffe in einer Presseaussendung ide orgaven Angrife der österreichischen Sicherheitsorgane gegen Journalistinnen und Journalisten bei ren wenige Tage zuvor und kündigten eine Meldestelle fürVerstöße gegen die Pressefreiheit in Öster-

- Es wird daher empfohDeutschland je nach Einsatz unterschiedliche $\mathbf{E r}$. kennungsnummern sichtbar an Uniformen ange-

Die

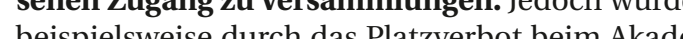
miker-Ballin Jahr geschränten Zugang der MedienverteterInn den Versammlungen und Gegenversmmlungen beschnitten (Interview 7). Von RechtsvertreterInnen und CSO-VertreterInnen wurde dies als ungerechtfertigt eingeschätzt (Interview 7 I2 I5 un I9). Die Berichterstattung war nur unter Polizeikontrolle für JournalistInnen (embedded journalism)

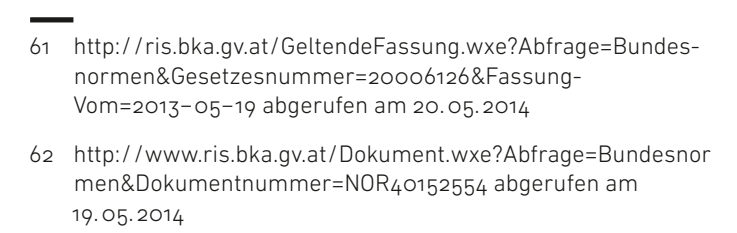

reich ${ }^{64}{ }^{64}$. Anlass für diese Maßnahme war die Entscheidung Wiener PolizistInnen JournalistInnen und einer Vertreterin der Volksanwaltschaft bei der Demonstration wiederholt den Durchgang im Bedes Volkstheaters zu verwehren.

Darüber hinaus kam die Kritik auf, dass Jouralistinnen aus vorauseilendem Gehorsam gegenMedieneigentümerInnen oder Anzeigenkunberichten. Im öffentlich manche Versammlungen unschenswert, wenn über politische Versamn lungen berichtet würde (Interview I5 und I9).

rsammenen, die in Zusammenhang mit 政

Im österreichischen Strafrecht (StGB) stehen diverse Bestimmungen in Widerspruch zur Meinungs- oder Versammlungsfreiheit. Diese Einschränkungen von zivilgesellschaftlichem Engage政 tisch für Organisationen ist der Vorwurf der Nö̈tigung $($ ( I 05 ff StGB). Wenn Kampagnenorganisationen Unternehmen mit dem Vorwurf der schlechten Unternehmensführung oder Umweltschädigung öffentlich unter Druck setzen, laufen Diese Paragraphen sind u a im Verfahren gegen TierschützerInnen (VGT) zum Einsatz gekommen. Außerdem wurde 2013 wegen Aufforderung mit Strafe bedrohten Handlungen und GutheiBung von mit Strafe bedrohten Handlungen $(\$ 282 \text { Absatz } 2 \text { StGB })^{65}$ gegen den Flüchtlingshelfer Michael Genner der CSO Asyl-in-Not ein Strafantrag gestellt. Er hätte seine vorsätzlich begangene, mit einer ein Jahr übersteigenden Freiheitsstrafe bedrohte Handlung in einer Art gutgeheißen, die geeignet ist, das allgemeine Rechtsempfinden zu empören oder zur Begehung einer solchen Handlung aufzureizenı. Dafur hätten ihm bis zu zwei Jahre Gefängnis drohen kônnen. ${ }^{66}$ Er habe öffentlich daraufhin gewiesen, dass Schlepper Menschen die Hucht vor Verolgung, Folter und Mord ermoglichen und so-

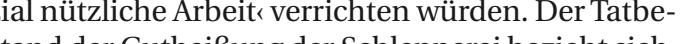
列 aus walscister 12) ${ }^{67}$ Die Oberstaatsanwaltschaft hat der Staatsanwaltschaft die Weisung erteilt, ihren strafantrag zurückzuziehen. ${ }^{68}$

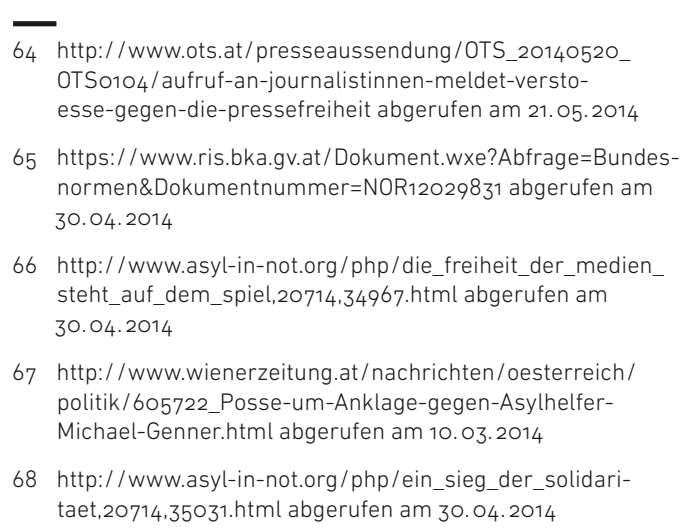




\section{ANALYSE ERR RAMMENEEDNGUNGEN}

VERBESSERUNGSPOTENZIALE

An die Adresse der Gesetzgebung Es können folgende Verbesserungspotenziale ideniniziert we

Eine explizite Anpassung des Versammlungsgesetzes an die Erfordernisse der EMRK und des IPBPR bei gleichzeitiger Rücknahme des Vorbehalts zum IPBPR.

. Konkret betrifft das z. B. die Möglichkeit von EU-BürgerInnen sowie von Drittstaatsangehörigen in Österreich Versammlungen abzuhalten, zu leiten oder als OrdnerInnen aufzutreten.

3. Aktuell gibt es in Österreich keinen zeitgerechten Beschwerdemechanismus, der es erlaubt, wegen eines ungerechtfertigten Untesagungsbescheids im Rahmen eines Beschwedeverfahrens sein Recht aufVersammlungsJeiheit noch vor dem anvisierten Versammlungstermin einzufordern. Eine Möglichkeit zur Verbesserung wären Eilverfah an Verwaltungsgerichten,

4. Maßnahmenbeschwerden gegen unverhältnismäßige Maßnahmen der Sicherheitsbehohen Kostenrisiko beiVerlust des Verfahren verbunden und werden nur selten genutzt. Das Kostenrisiko sollte reduziert werden, um Menschen mit geringem Einkommen nicht von der Möglichkeit eine Maßnahmenbeschwerde einzureichen abzuhalten

. Um das Fehlverhalten einzelner PolizistInnen tatsächlich ahnden zu können, wird empfohlen, dass ähnlich wie in anderen Ländern, z. B. Deutschland, je nach Einsatz variierende Erkennungsnummern sichtbar an Uniformen angebracht werden (Interview I7).

. Das Prinzip der Deeskalation soll bei der Vorbereitung und Durchführung von Einsätzen bei Versammlungen systematisch veranker werden. Großräumige Platzverbote und eskalatives Vorgehen wie Kesselbildung und Einsatz von Gewalt bei Versammlungen sind unbedingt zu vermeiden.

Verwaltungsstrafen gegen Versammlungs teilnehmerInnen sollen nur in rechtlich begründeten Fallen und nicht als repressive

Maßnahmen eingesetzt werden.

. Etwaiges Fehlverhalten wie die nicht-verhältPolize soll duch poliziin terne Kontrollen erfasst werden. Die Feststex-

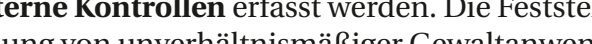
dung soll für die handelnden Personen Konsequenzen durch die Disziplinarbehörde bedeuten, die eine tatsächliche Präventivwirkung entfalten.

9. Die Videoüberwachung von Kundgebungen soll tatsächlich nur bei Gefährdung der öffentlichen Sicherheit und Ordnung eingesetzt werden.
Strafrechtliche Vorwürfe des ,Landfriedensbruch` ( $\$ 284$ StGB), der /Nötigung`( $($ I05 ff StGB) und die AAufforderung zu mit Strafe bedrohten Straftaten und Gutheißung von mi Strafe bedrohten Straftaten ( $\$ 282$ StGB) solle abgeschafft oder zumindest so modifiziert werden, dass sie keine Möglichkeit bieten zivilgesellschaftliches Engagement zu kriminalisieren (Interview 7, I2 und I8).

Das Recht auf Versammlungsfreiheit soll auch für Menschen ohne österreichische Staatsbürgerschaft, egal welcher Herkunft, in nationales Recht umgesetzt werden.

Grundsatzlich soll das Wissen um Relevanz und Bedeutung der Versammlungsfreiheit in der gesamten Bevölkerung gestärkt werden. Das Verstănnnis und Wissen der Bevôlkerung über ihre eigenen Rechte, das Wesen und die Wirkungsweise politischer Versammlungen is in einer Demokratie unerlässlich. Eine Mögilchkeit dafur ist dem Versammlungsrecht im Rahmen der politischen Bildung an Schule eine starke Bedeutung zu geben.

Gebieten soll das Wissen der Sicherheitsbe-

ïn freiheit und damit zusammenhängenden

Modalitäten gestärkt werden, um Fehlentsche-

dungen und damit Eingriffen in die Versamm-

ungsfreiheit, wie unzulässige Untersegungen

von Versammlungen, vorzubeugen

Unbedingter Schutz und Gewährleistung der

Pressefreiheit durch die Exekutive.

An die Adresse der CSOs und

Um bei Sicherheitsbehorden nicht den Eindruck der Gefährdung der öffentlichen Sicherheit und Ordnung zu erwecken und damit epressives Vorgehen der Behörden (Platzverbote, Untersagungen) zu provozieren, wird empfohlen in der Kommunikation vor und während Versammlungen auf Gewaltandrohungen zu verzichten.

Das Verständnis und Wissen innerhalb des CSO-Sektors über Versammlungsfreiheit und die damit verbundenen politischen Rechte sind ausbaufahig. Best-Practice Modelle wie Aktionsakademien, Handbücher, Sen
sollten verstärkt umgesetzt werden. Die Medienberichterstattung über VersammRolle. Eine politische Kultur in derVersa lungen als ein wertvoller Bestand teil einer demokratischer

werden ist eine wichtige Voraussetzung fü

Active Citizenship (Interview I5). Strategische Kommunikation der Anliegen Ursachen und Notwendigkeit von Protestaktionen sollte integraler Bestandteil von Versammlungen werden.

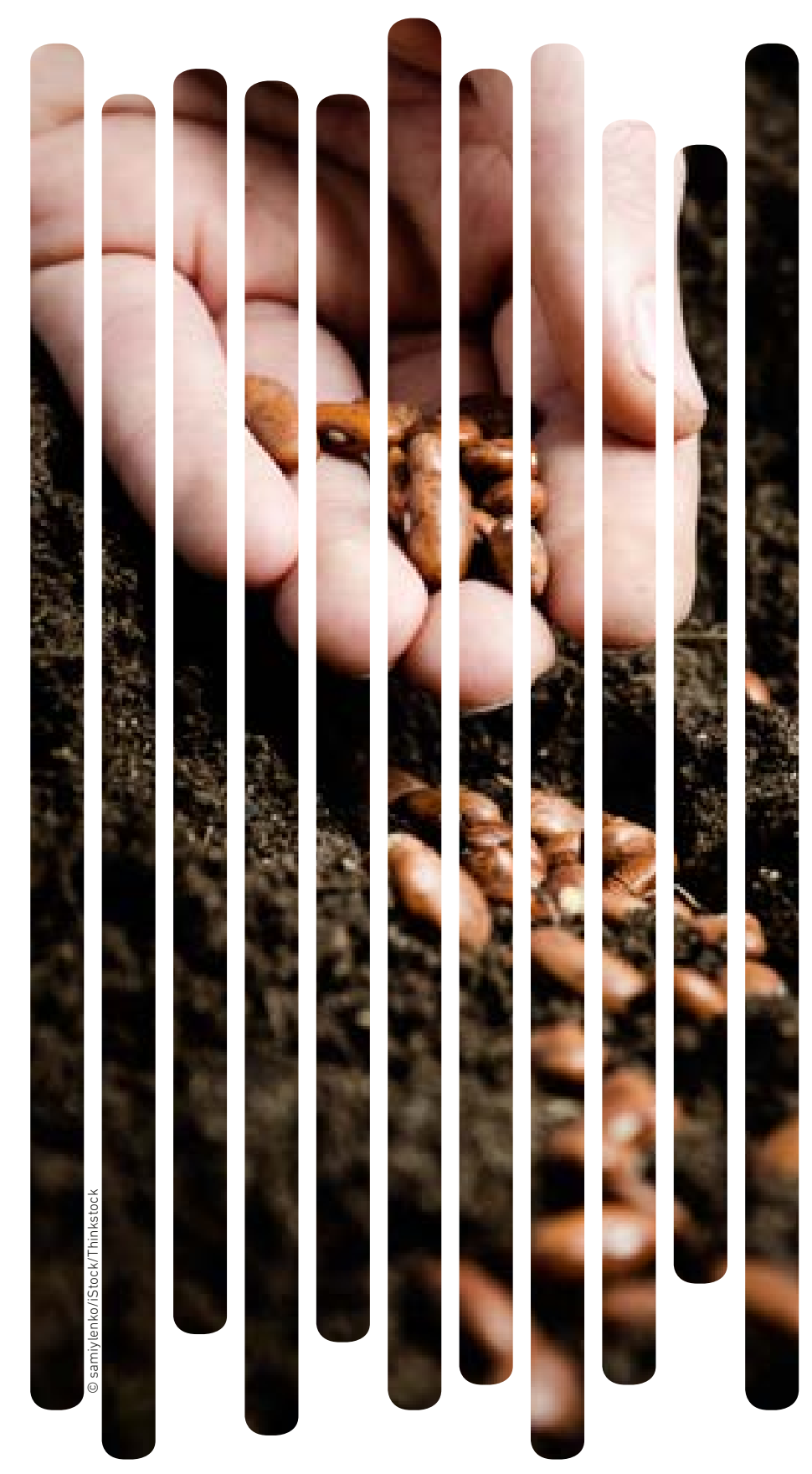

CSOs übernehmen öffentliche Dienstleistungen und decken soziale Dienstleistungsbedarfe ab, die weder vom Markt noch von der öffentlichen Hand in der benötigten Menge bzw. Qualität angegesellschaftliche Rolle, gleichzeitig sind sie oft mit finanziellen Unsicherheiten und Herausforderungen konfrontiert. Diese bestehen darin, durch einen geeigneten Finanzierungsmix soziale und wirtschaftliche Ziele miteinander zu verknüpfen. Wahrend rund $53 \%$ der Einnahmen von CSOs von der öffentlichen Hand und $36 \%$ aus Markteinnahmen stammen, werden rund I I \% der Einnahme durch Spenden und Sponsoring aufgebrach Schneider et al.

\section{RECHTLICHE REGELUNGEN}

\section{RECHTHCHE REG HUNGEN-INDIKATORE}

1. Die Gesetzgebung ermöglicht es CSOs, wirtschaftlich als
Marktanbieter tätig zu sein.
2. CSOs dürren finanzielle Mittel aus dem Ausland annehmen.
3. CSOs dürfen finanzielle Mittel, z. B. Finanzierungen von Ein-
zelpersonen, Unternehmen und anderen Quellen erhalten.
Die Gesetzgebung in Österreich unterscheidet Organisationen, wenn es darum geht, als Marktanbieter wirtschaftlich tätig zu sein. Je nach Aufgaben und Funktionen der CSOs gestalten sich die Mög lichkeiten, Markteinnahmen zu generieren jedoch sehr unterschiedlich. Markterlöse spielen vor allem bei CSOs eine Rolle, die Dienstleistungen anbieten wie z. B. Leistungsentgelte, die bei Selbstbehalten (z. B. bei Pflegeleistungen) anfallen, Gebühren (z. B. Museumseintritte) sowie kostendeckende Einnahmen aus dem Verkauf von missionsbasierten Leistungen (z. B. private nicht gestützte Pflegeberatung). Typisch für CSOs ist dabei, dass EmpfangerInnen und ZahlerInnen einer Leistung häufig nicht ident sind, wie etwa bei Leistungsverträge mit der öffentlichen Hand, Transfers von anderen CSOs oder Sponsoring bzw. Unternehmenspartnerschaften im Rahmen von Corporate Social Responsibility. NPOs mit der Funktion der Interessenvertretung und/oder Gemeinschaftsbildung haben im Gegensatz zu Dienstleistungs-NPOs meist keine

4. Zugang zu finanziellen Ressourcen

n diesem Abschnitt wird die Frage aufgeworfen, ob zivilgesellschaftliche Organisationen (CSOs) in Österreich ihre finanziellen Mittel aus verschiedenen in- und ausländischen Quellen frei suchen und sichern können. Beim Thema Finanzierung unterscheiden sich CSOs deutlich von gewinnorientierten Organisationen. CSOs verfolgen gemeinnützige Ziele und sind gemäß Statuten oder Förderbedingungen nicht berechtigt, erwirtschafte Überschüsse an die EigentümerInnen auszuschütten. Finanzierungsaufgaben sind daher Mittel zum Zweck und werden gerade bei kleineren Organisationen oft als notwendiges Übel betrachtet, da eine hohe Identifikation mit ihren inhaltlichen Aufgaben oder der Mission im Vordergrund steht. Die Einkünfte aus Spenden oder öffentlichen Förderungen lassen sich nicht mit hoher Planungssicherheit vorhersagen. Es
dominieren daher Überlegungen zur Aufrechterhaltung der Liquiditât, Fragen der Rentabilität sind eher sekundär (Littich/Schober 2013).
Geren gine Unabhänhre Einkünfte oft nur aus Mitgliedsbeträgen und Littich/Schober 2013).

en hinsichtlich ihrer Finanzieungsquellen unterschiedlich stark diversifiziert sin. Es gibt CSOs, die lediglich eine Finanzierungsquelle (z. B. Mitgliedsbeiträge, Förderungen) nuten, aber auch solche, die mehr als zehn Finanzieungsquellen haben. Durchschnittlich nutzen CSOs in Österreich 5,2 Quellen (Schober et al. 20II). Die Kunst der Finanzierung liegt in der Aufrechterhaltung der Liquidität auch abseits von Markterlöen. ${ }^{69}$ In Österreich spielt hierbei die öffentliche and als Financier eine bedeutende Rolle. Bei einer ohen Abhängigkeit von Mitteln der öffentlichen
and, können Einschränkungen der staatlichen

bilden. Darüber hinaus kann die stenerlich Jahresbudgels bilden. Darüber hinaus kann die steuerliche Begünstigung
aberkannt werden, wenn keine großen Investitionsvorhaben 


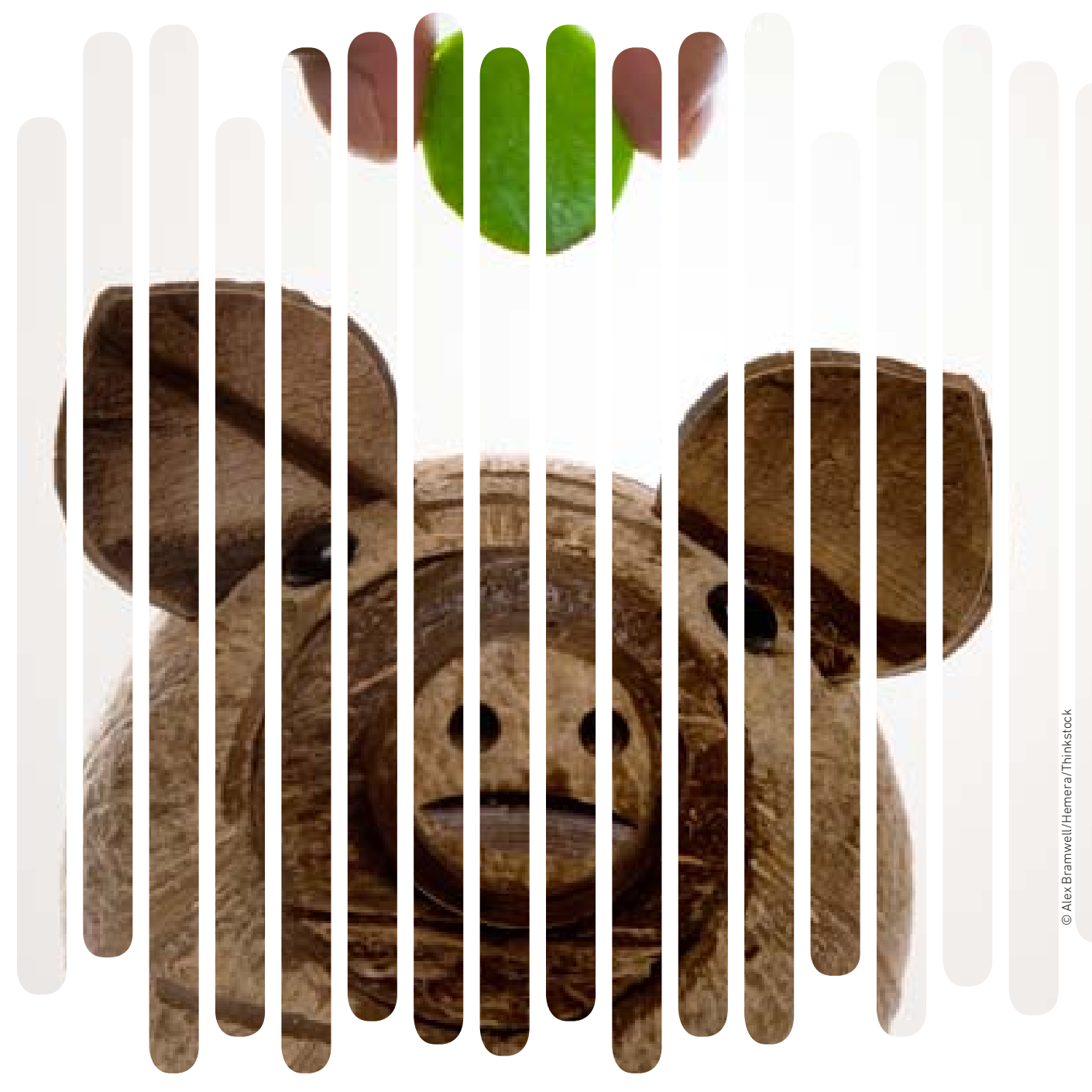

Ausgaben Probleme bei der Aufrechterhaltung des blieren, was auch im Regierungsprogramm Leistungsangebots zur Folge haben. Problematisch 2013-2018 verankert ist ${ }^{\text {to }}$.

sind auch mitunter übliche Regelungen in Förder- Bei Finanzierungen durch Spenden kann von verträgen und Verordnungen, wonach Einnahmen unterschiedlichen Quellen ausgegangen werden. abseits von öffentlichen Förderungen letztere um Die Spenden können von Einzelpersonen, Unterdiesen Betrag reduzieren. Dadurch wird der Hand- nehmen oder Stiftungen kommen. Das öffentliche lungsspielraum der CSOs stark eingeschränkt. Spendensammeln ist in Österreich Ländersache Alternative Finanzierungsquellen können aus und durchwegs bewilligungspflichtig. Spendenaufdem Bereich (Venture)-Philanthropie und zum rufe, die auf dem Postweg oder über die MassenTeil von gemeinnützigen Stiftungen stammen. medien erfolgen, sind von der Bewilligungspflicht Grundsatzlich werden unter Venture Philanthropie ausgenommen. Auch für Sammlungen von Gebiets Großspenden von vermogenden Privatpersonen körperschaften, politischen Parteien, Kirchen, mittelt, verstanden (Pepin 2005). Die Gebenden in- hen die Sammlungsesetze Ausnahmen vor, die volvieren sich hierbei oft weit stärker als her- sich allerdings von Bundesland zu Bundesland unkömmliche SpenderInnen und entscheiden über - tercheiden. Grundsötzlich bewilligungspflichtig ist die Mittelverwendung mit Derzeit werden in Öster - diëffentliche Mitoliederwerbungwenn etwa nach reich zwischen I- 5 Euro pro Jahr und Einwohner der Art und dem Umfang der Aufforderung oder aus Stiftungen für gemeinnützige Zwecke aus- den sonstigen Umständen anzunehmen ist dasses geschüttet. Dieser Beitrag ist äußerst gering im Ver- sich hiebei nicht ernstlich um die Herbeiführung eigleich zu beispielsweise Deutschland (I80-230 nes dauernden Verhältnisses zum Verein, sondern Euro) (Schneider et al. 2010). Dies liegt u. a. an steu- vielmehr nur um die Erlangung von Geld oder von errechtlichen Rahmenbedingungen, die zwar anderen Leistungen handelt ( $\$ 2$ Abs 4 NÖ SammPrivatstiftungen ermöglichen, aber eher auf eigen- lungsgesetz). Die Anwerbung von fördernden Mitnützige Stiftungen gemünzt sind. In Osterreich gibt gliedern unterliegt dann nicht den Sammlungsgees derzeit Bestrebungen einen (eigenen) rechtli- setzen, wenn ihre Rechte und Pflichten in der chen Rahmen für gemeinnützige Stiftungen zu eta- Satzung klar festgelegt sind, wobei es durchaus zu- lässig ist, wenn der vorrangige Zweck der Mitgliedschaft in der Leistung finanzieller Zuwendun besteht. ${ }^{71}$

Im Allgemeinen dürfen CSOs finanzielle Mittel aus dem Ausland annehmen. Größere, international tätige Organisationen errichten zu diesem Zweck meistens Zweigvereine in den jeweiligen Ländern. Aber auch die Annahme von Spenden aus dem Ausland durch nur in Österreich tätige CSOs ist unproblematisch. Nach dem EU-Recht sind Spenden nicht vom Anwendungsbereich der Niederlassungsfreiheit erfasst, jedoch unterliegen Spendentransfers der Kapitalverkehrsfreiheit. Der Grund ist, dass Spenden mangels eines Entgeltcharakters keine Erwerbstatigkeit begründen (Achatz 2006). Laut $\$ 40 \mathrm{Abs.I} \mathrm{Z2} \mathrm{BWG}^{22}$ sind in Österreich (zur Bekämpfung von Geldwäscherei und Terrorismusfinanzierung) Einzahlungen ab I5.00o Euro zu identifizieren. Die Finanztransaktionen innerhalb des europäischen Raumes können mittels SEPA Uberweisungen problemlos abgewickelt werden Außerhalb des Euro-Raumes wird fur großßere Be träge i.d.R. aut Devisentransaktionshandler, wie Wirvern ledigen (Interview 20)

Bei Spenden aus dem Ausland kommt es manchmal zu Problemen, wenn der/die SpenderIn die Spende von der Steuer absetzen will Dazu muss die begünstigte CSO die dafür notwendigen Voraussetzungen im Ursprungsland der Spende erfüllen Da es in Österreich keinen Feststellungsbescheid über die Gemeinnützigkeit gibt (s. Kapitel 5), bedeutet dies eine Einschränkung für Spenden aus dem Ausland. Betroffene CSOs greifen deshalb manchmal auf den Spendenbegünstigungsbescheid oder das Spendengütesiegel zurück, um indirekt die Gemeinnützigkeit nachzuweisen, das gelingt aber nicht immer (Interview 20).

71

Rechtsgutachten ,öffentliches Sammeln und Fördermitgliederwerbungs, erstellt von o. Univ.-Prof. Mag. Dr. Bernd-
Christian Funk im Auftrag des Fundraising Verbandes Austr Mai 2010

72 http: / / www.ris.bka.gvat/GeltendeFassung.wxe?Abfrage=

\section{BESONDERHEITEN IN DER PRAXIS}

(III) PRAXIS-INDIKATOREN

implementiert und sind nicht diskriminierend für

Es gibt keine Einschränkungen (z. B. Hürden bei der Verwaltung oder Finanz, oder Kanalisierung von Fonds über spezifische Stellen) für den Erhalt von ausländischen Finanzmitteln

Der Erhalt von finanziellen Mitteln von Einzelpersonen, 列 .

Bei Spendenfinanzierung von Einzelpersonen, Unernehmen und Stiftungen kann grundsätzlich wischen freien und zweckgebundenen Spenden unterschieden werden. Es lässt sich ein Trend be Spendenorganisationen zu zweckgebundenen Spenden beobachten (Interview 20). Stiftungen 列 Zweckwidmung bestimmte Projekte unterstützen. Z Von Zweck der Spendenorganistion übecinstimnt Zweck der Spendenorganisation übereinstimm ungsceber wie der Austrian Development AnzieADA) verfolgt wird. Dadurch werden Organisationen weniger flexibel und sind auch in ihrer Liquidiät eingeschränkt. Die SpenderInnen sind in der Regel bekannt, da ein Interesse besteht, eine Bestätigung für die Spendenabzugsfähigkeit zu erhalten (Interview 20).

Im Allgemeinen haben CSOs einen breiten Zugang zu Finanzierungsmöglichkeiten, allerdings bestehen Schwierigkeiten bei der Aufnahme von Fremdkapital. Bei Kreditfinanzierungen sind jene begünstigt, die úber regelmäßige und nachhaltige Einnahmen verfügen (z. B. Mitgliedsbeiträge eines Autofahrerklubs), eine kontinuierliche Finanzierung durch die öffentliche Hand aufweisen und/oder stärker diversifiziert sind (Yan et al. 2009). Nachdem herkömmliche Banken bei der Bewertung der Kreditwürdigkeit und Kreditfähigkeit von CSOs Probleme haben, etablieren sich zunehmend Spezialbanken nach dem Social Banking Modell, die fur CSOs tatig sind. Die GLS-Bank aus Deutschland z. B. hat erstmalig in Osterreich das Wohnprojekt Win auf den Nordbahn Dabei wiste die Enwroum in wochwertigen Form eines partiziptiven Prozess fingum in terstïtzt In Österreich ist lie Erste Bonkim Bereich Social Banking aktiv und versucht Finanzierungen für CSOs jenseits des herkömmlichen Rating Systems bereit zu stellen. 2014 nimmt auch die Bank für Gemeinwohl in Österreich konkrete Formen Diese erste zivilgesellschaftliche Bank soll besonders günstige Kredite für sozial und ökologisch nachhaltige Projekte ermöglichen

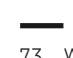

74 (http://www mitgruenden at abgerufen am 12042014 
Einerseits gibt es Vorschriften, die die wirtschaftlichen Aktivitäten von begünstigten CSOs positiv diskriminieren, aber auch andererseits Grauzonen die CSOs in der Praxis negativ diskriminieren. Im Besonderen ist die Finanzierungform des Crowdfundings zu erwähnen. Die österreichische Finanzmarktaufsicht (FMA) ermittelt nach Medienberichten in ungefähr 39 angezeigten Fällen in Sachen illegaler Finanzmarktgeschäfte, von denen einige auch CSOs betreffen, die zinsenlose Privatdarlehen aufgenommen haben. ${ }^{75}$ Derartige Geschäfte werden von der FMA als gesetzeswidrig eingestuft, da andernfalls ein nicht regulierter Kreditmarkt entsteht. Die FMA wurde in
einem Präzedenzfall in ihrer Rechtsmeinung bestäeinem Präzedenzfall in ihrer Rechtsmeinung bestätigt, dass es sich dabei um ein Bankgeschäft handel für welches eine Bankkonzession benötigt wird. Betroffen sind vor allem KMU aus der Konsumgüterund Solarenergiebranche. In einigen Fällen habe sich CSOs mit der FMA auf das legale Finanzie-
rungsmodell Nachrangsdarlehen geeinigt. Damit rungsmodell Nachrangsdarlehen geeinigt. Dam haben private Anleger im Falle eines Konkurses im gen Anspruch auf die Konkursmasse 75 .

Im Wesentlichen ibt es in der Praxis bei nanztransaktionen aus dem Inland und Ausland Rei Spenden aus somit keine Einschränkungen. Beträge mit Kreditkartenunternehmen abt keinere bei welchen Spesen entstehen. In allen anderen $F$ ällen kommen Banküberweisungen zum Finsatz $\mathrm{Fie}$ für CSOs oft zu vergünstigen Konditionen angebo ten werden. Wie bereits angesprochen, können Schwierigkeiten mit der Absetzbarkeit und dem fehlenden Nachweis der Gemeinnützigkeit entstehen, wenn Spenden aus dem Ausland entgegengenommen werden (Interview 20). Eine weitere Einschränkung betrifft die Liquidität und die Veranlagung von Finanzmitteln. Gerade spendenfinanzierten CSOs sind gewisse Grenzen gesetzt, da in der Veranlagung keine risikoreichen Formen zu verantworten sind, d. h. das Lukrieren hoher Zinsen aus Aktien, Derivaten und Optionen ist nicht möglich. Daher werden nachhaltige und risikoarme Veanlagungen sowie Vorteile bei Banken durch ver-
günstigte Konditionen oder bessere Zinssätze genutzt (Interview 20).

Damit Spendenflüsse von Einzelpersonen, Unternehmen und der öffentlichen Hand über ein längere Zeit erhalten bleiben, benötigt es sowohl
Anreize zum Spenden als auch Kontrolle bei der Anreize zum Spenden als auch Kontrolle bei der
Verwendung von Spenden. In Österreich hat erst kürzlich die Capital Bank eine Gemeinschaftsstiftung gegründet, die sich zum Ziel gesetzt hat, vermögende SpenderInnen und gemeinnützige Projekte zusammenzubringen. ${ }^{77}$

\section{backlink=/home/Wirtschaft//economist/supermarkt// $1316460 /$ index.d.o8s
am 12.04.2014.

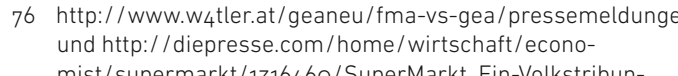

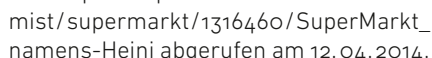 .}

77 http://diepresse.com/home/meingeld/1593769/Richtigspenden-muss-gelernt-sein? VLLbacklink=/home/w

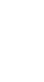

Für viele spendensammelnde CSOs ist die Anwerbung von fördernden Mitgliedern im öffentlichen Raum ein wichtiges Mittel zur Diversifizierung ihrer Einkünfte. Bei manchen CSOs stammen bis zu $80 \%$ ihrer Spendeneinnahmen aus Fördermitgliedschaften. Sie beklagen daher die restriktive Anwendung der Sammlungsgesetze in manchen Bundesändern. Die Bundeshauptstadt Wien hat 2014 eine Entscheidung des Unabhängigen Verwaltungssenats und einem Gutachten des Verfassungsjuristen Bernd Funk Rechnung getragen und das Wiene Sammlungsgesetz entsprechend abgeändert. Andere Bundesländer, wie Niederôsterreich und Kärn-

.

\section{VERBESSERUNGSPOTENZIALE}

Folgende Verbesserungspotenziale wurden von den

viewpartnerInnen genannt:

Verbesserungsbedarf besteht bezüglich der Rechtssicherheit beim Erhalt von finanziellen Mitteln aus dem Ausland oder bel Finanzierungsformen wie Crowdfunding. Ein Bescheid, der die möglichen, vernis cenerier (Interiew (so) (sihe auch Kapiel Stergeneront)

Wenn im vorherrschenden Finanzierungsmix dann muss durch Alternativen Gelder zurückgehen, werden. Förderklauseln, die bestimmen dass die Förderungen bei alternativen zusätzlichen nahmen um den entsprechenden Betrag reduziert werden, sind kein Anreiz zum Ausloten alternativer Finanzierungsformen.

Da der Zugang zu Fremdfinanzierungen für CSOs durch stärkere Fokussierungen auf Eigenkapital bei Kreditwerbern (Basel III Bestimmungen) immer problematischer wird, können Social Banking Modelle, wie in Osterreich etwa durch die Gründung der Gemeinwohl Bank, weiter untertzt werden.

Darüber hinaus sollen auch andere AktivitäCrowdfunding oder die Änderung des Stiftungsrechts den Zugang zu finanziellen Ressourcen ver-

Schließlich drängen spendenfinanzierte CSOs auf die Herstellung der Verfassungskonformitât in den jeweiligen Sammlungsgesetzen der CSOs dereit wh po ine Forderes (

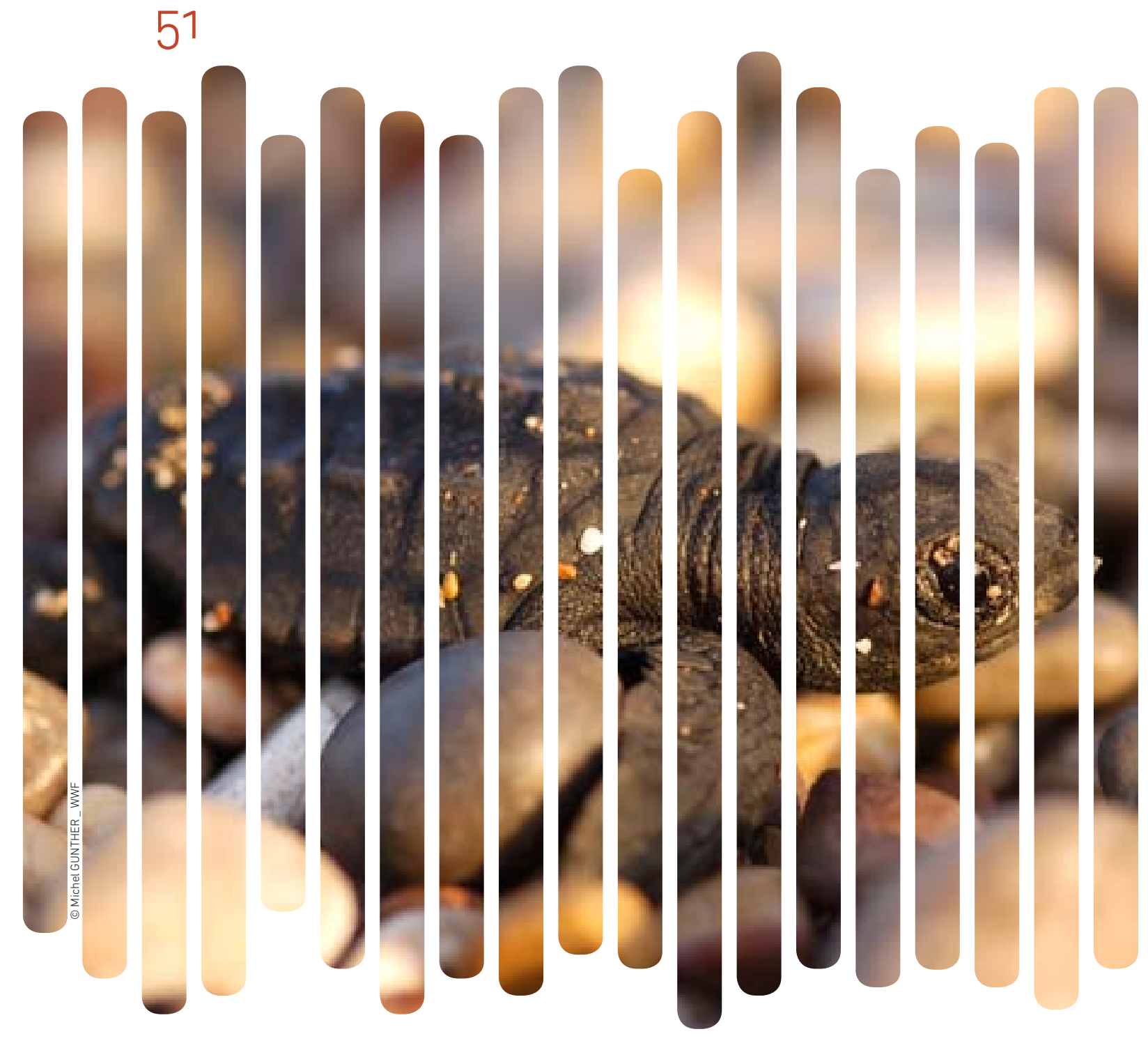

5. Steuerrecht

m Allgemeinen herrscht in Europa ein gesellschaftlicher Konsens darüber, dass CSOs wegen ihrer Leistungen für die Allgemeinheit gegenüber gewin-
norientierten Unternehmen steuerlich begünstigt norientierten Unternehmen steuerlich begünstigt werden sollen. Form und Umfang der Begünstigung können sich jedoch von Land zu Land stark unte scheiden und im Lauf der Jahre verandern (Friedrich et al. 2005). Das österreichische Steuerrecht,
dessen Ursprünge bis in die Monarchie zurückreichen und das mit dem Abgabenrecht der Bundesrepublik Deutschland viel gemeinsam hat, hat sich im Gegensatz zu diesem, was die Gemeinnützigkeit angeht, in den letzten Jahrzehnten wenig verändert ${ }^{78}$ Nach Ansicht aller von uns befragten ExpertInnen ist eine Reform des Gemeinnützi keitsrechts in Österreich daher seit langem übefällig.

\section{$\overline{78} \mathrm{D}$}

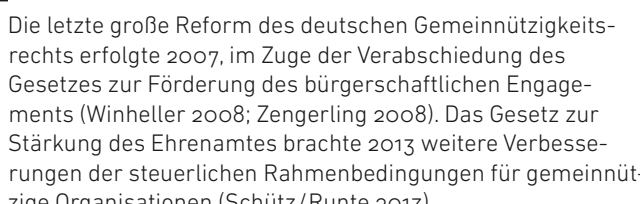

Obwohl das Steuerrecht grundsätzlich Sache sierungsbestrebungen der Europaisschen Kommission auch betreffend die steuerliche Begünstgung von CSOs, etwa mit dem Vorschlag für eine veras oder der Oberprufung beste-

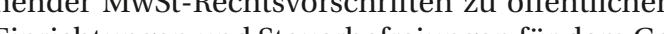
ind nationale Gesetzgebung sind allerdings derzeit ch nicht absehbar.

Grundlage für diesen Teil des Berichts sind die schriftlichen Rückmeldungen von Io Wirtschaftauf eine Online-Befragung und Interviews mit 7 ausgewiesenen GemeinnützigkeitsexpertInnen im Zeitraum März bis April 20I4.

$$
\begin{aligned}
& \text { http://ec.europa.eu/internal_market/company/eufounda- } \\
& \begin{array}{l}
\text { http://ece.europa.eu/taxation_customs/resources/docu- } \\
\text { ments/common //consultations/tax/public_bodies/consulta- } \\
\text { tion_document_de.pdf }
\end{array}
\end{aligned}
$$
streuhänderInnen und PraktikerInnen aus CSOs 


\section{RECHTLICHEREGELUNGEN-INDIKATOREN} Steuerbegünstigungen für CSOs sind vorgesehe

Zuschüsse/Subventionen/Zuwendungen und Spenden vor, die nicht gewinnorientierte Aktivitäten von CSOs unterstützen

2. Das Gesetz sieht $S$ 3. Das Gesetz sieht Steuerbegünstigungen für passive Anlageformen (Vermogensverwaltung) von CSOs vor.

teile für (gemeinnützige) Stiftungen.

Es besteht ein hohes Maß an Rechtssicherheit in Bezz die gemeinnützigen Aktivitäten von CSOs.

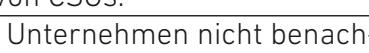

\section{Absetzbarkeit von Spenden}

\section{Das Gesetz sieht Steuerbegunstigungen für private und}

Un

Es gibt klare Vorgaben/Bedingungen für den Erhalt abzugslahiger Spenden und dese umf

Die für CSOs wesentlichen steuerlichen Bestimmungen sind insbesondere in dem Bundesgesetz ber allgemeine Bestimmungen und das Verfahre Lür die von den Abgabenbehörden des Bundes, der desabgabenordnung - BAO), BGBl. Nr. I94 / Bu zuletzt geändert durch das Abgabenänderungsgesetz 20I4, enthalten. Das Gesetz legt die allgemeinen Voraussetzungen fest, die gegeben sein müssen, damit eine CSO steuerlich begünstigt werden kann. Dazu muss sie neben einigen formalen Anforderungen jedenfalls gemeinnutzige, mildtatige und Satzung, Stiftungsbrief oder ihrer sonstigen Rech grundlage und nach ihrer tatsăchlichen Geschäfts政 rung der

ie BAO unterscheidet verschiedene Tätigk en ('Sphärens) einer CSO: Unentgeltliche Leistungen sowie Einnahmen, z. B. Spenden, denen keine unmittelbaren Gegenleistungen gegenüberstehe (svereinssphäres) werden von der Vermögensverwaltung, wirtschaftlichen Geschätsbetrieben ohne Gewinnabsicht und - grundsätzlich begünstigungs-

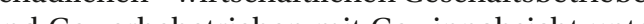

Wirtschaftliche Geschäftsbetriebe werden der als unentbehrlich, enthehrlich oder beginn gungsschädlich eingestuft und unterschiedlich steuerlich behandelt Unentbehrliche Hilfsbetriebe dürfen nur `Zufallsgewinner erwirtschaften und nicht zu abgabepflichtigen Betrieben derselben oder ähnlichen Art in größerem Wettbewerb stehen.

\section{3}

Hier liegen die größten Gefahren für die CSOs, da ein begünstigungsschädlicher wirtschaftlicher $\mathrm{Ge}$ schäftsbetrieb automatisch Steuerpflicht und deSchätsbetrieb automatisch Steuerpflicht und de fern nicht von einem individuellen oder generelle Ausnahmerecht Gebrauch gemacht wird (Achatz Oberleitner 20I3).

Obwohl die BAO seit I96I gezählte 86-mal novelliert wurde, sind die für CSOs maßgeblichen $\$ \$ 34 \mathrm{ff}$. seither praktisch unverändert geblieben. Um den Veränderungen der letzten 50 Jahre im dritten Sektor dennoch Rechnung zu tragen, haben die österreichischen Abgabenbehörden eine umfan reiche Verwaltungspraxis und Judikatur entwickel und in den Vereinsrichtlinien dokumentiert. Diese werden regelmäßig adaptiert und grundsätzlich auf alle Körperschaften, also auch auf gemeinnützig Stiftungen und auf gemeinnützige Kapitalgeselschaften $(\mathrm{GmbH})$ angewendet. ${ }^{\text {8r }}$ Sie sind aber fü die Abgabenbehörden und Gerichte nicht verbin lich, da sie weder eine Verwaltungsanweisung noch eine Verordnung darstellen. Rechtssicherheit, wie in Dever inch

Art und Umfang der steuerlichen Begünstigungen sind in den jeweiligen Abgabengesetzen (im Körperschaftssteuer- $(\$ 5$ Abs 6 KStG), Umsatzsteuer- ( $\$$ Io Abs 2 Z 7 UStG), Kommunalsteuer- ( $\$ 8 \mathrm{KommStG})$ und Grundsteuergesetz ( $\$ 2 Z_{3}$ lit b und $Z_{4}$ GrStG) geregelt. Von de Kommunalsteuer sind unternehmerisch tätige CSOs nur dann befreit, wenn sie mildtätigen Zwecken oder gemeinnützigen Zwecken auf dem Gebie der Gesundheitspflege, Kinder-, Jugend-, Familienken-, Behinderten-, Blinden- und Altenfur-

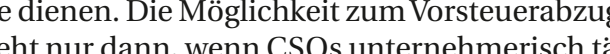
tonn (Kent) liegen der Kapitalertragsteuer (KESt) (\$ I Abs $3 Z_{3}$ dann möglich (und sinnon der KESt-Pflicht ist nu dann moglich (und sinnvoll), wenn die Kapitalanlagen notwendiges Betriebsvermogen eines wirtdessen Verluste die Kapitalerträge übersteigen) (Achatz/Oberleitner 2013). Gemeinnützige Rechtsträger, die ihre gemeinnützige Tätigkeit nur über finanzieren, sind dadurch benachteiligt.

Eine Sonderstellung haben in Österreich die Sportvereine, die an Sportler, Schiedsrichter und Spo Euro pro Tag bzw maximal 540 Euro pro Kan dermonat steuer- und sozialversicherungstei auszahlen dürfen ( $\$ 3$ Abs. I Z IOc EStG und $\$$ 49 AbsZ 9 AVG) Andere 1 in ing Einrichtungen, die Rettungsorganisationen einge-

Die Vereinsrichtlinien haben im Lauf der Zeit einen von Laien
kaum mehr zu überschauenden Umfang angenommen: das

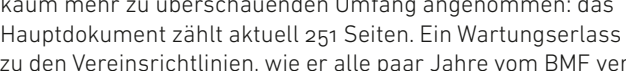
öffentlich wird, kann schon einmal über r 100 Seiten umfassen. schlossen, haben diese gesetzliche Möglichkeit die Abzugsfähigkeit auf ausländische Einrichtunnicht. Sportvereine und die Bergrettung sind unter bestimmten Voraussetzungen auch von derWerbeabgabe im Zusammenhang mit dem Sponsoring durch Unternehmen befreit (Achatz/Oberleitne 2013).

Auch für Körperschaften öffentlichen Rechts, wie die Kirchen oder die Freiwilligen Feuerwehren gelten eigene Regelungen, auf die aber hier nicht näher eingegangen wird.

Gemeinnützige Stiftungen spielen in Österreich, wie bereits an anderer Stelle festgestellt, nur eine untergeordnete Rolle. Für sie maßsgeblich sind das Bundesstiftungs- und Fondsgesetz und die diversen Landesstiftungsgesetze. Auch nach dem Privatstiftungsgesetz gibt es die Möglichkeit, teilweise oder ausschließlich gemeinnützige Stiftungen zu errichten. Nur einige wenige Stifter machen allerdings bisher Gebrauch davon (Schneider et al. 2010). Einer der Gründe dafur ist diegeforderte ausschließliche und unmittelbare Zweckerfuillung, welche poten zielle StifterInnen dazu zwingt, den jeweiligen ge meinn autren, anstatt in dem jewriligen Berich orfahrene ${ }^{2}$. Hindernis ist, dass Zuwendungen zu gemeinneïzigen Privatstiftungen steuerlich nicht begünstigt bzw. gegenüber anderen gemeinnützigen oder milddätigen Rechtsträgern sogar benachteiligt sind: Z dungen zu gemeinnützigen Stiftungen sind bei der Errichtung nicht abzugsfähig, da nur Einrichtungen spendenbegünstigt sind die bereits seit mindesten drei Jahren bestehen (FVA 2013b).

Grundsätzlich sind CSOs steuerlich gegenGer Unternehmen nicht benachteiligt. Es lässt sich aber eine Tendenz in der Steuergesetzgebung erkennen, CSOs mit Profit-Unternehmen gleichzustellen. Dies war etwa bei der Einfuhrung der Immobilienertragsteuer 20 I2 der Fall, von der gemeinnützige und mildtatige Organisationen nicht ausgenommen sind, auch wenn es sich um Veräußerungen von Immobilien infolge von Schenkungen oder Erbschaften handelt. Das gleiche droht aktuell be der anstehenden Reform der Grunderwerbsteuer, wo in Zukunft anstelle des bisherigen dreifachen Einheitswertes der i. d. R. sehr viel höhere Verkehrswert für die Berechnung herangezogen werden soll. In dem vorliegenden Gesetzesentwurf sind nur Ausnahmen fur Uberagungen innerhalb eines FamiliBis vor wenigen Jahren gab es in Österetch

Bis vor wenigen Jahren gab es in Osterreich Spendenabetbatkit) pratisch nur feir Spenden Spen wis auch feimillige Zuwendung an Organisationen, die entweder mildtätige Zwecke verfolgen oder ii Bereich der Armutsbekämpfung in Entwicklungsländern oder Katastrophenhilfe tätig sind absetzbar Seit 2012 sind auch Umwelt- und Tierschutzorganisationen spendenbegünstigt, letztere jedoch nur, sofern sie Tierheime betreiben. Außerdem wurde im Bereich der Forschung und Lehre ausgedehnt, sofern sie einer begünstigten inländischen orschungs- oder Bildungseinrichtung entsprechen. 2014 hat Österreich die Spendenabsetzbarkei bermals auf bestimmte Einrichtungen mit Sitz in einem Staat, mit dem eine umfassende Amtshilfe esteht, ausgeweitet. Dies betrifft insbesondere Museen und Einrichtungen im Bereich Kunst und a un 1988). Der österreichische Gesetzgeber re gierte damit auf ein EuGH-Urteil aus $201 \mathrm{I}$, das den Ausschluss dieser ausländischen Einrichtungen von 政 . im Be(a) usgenommen.

Voraussetzung für die Abzugsfähigkeit von Vist die Austellung eines Spendenbegün gungsbescheides durch das Finanzamt Wien I / 23 die darauffolgende Aufnahme in die beim BM führte Liste der spendenbegünstigten Einrichngen. Die spendenbegünstigte CSO muss zu diem Zeitpunkt bereits seit mindestens drei Jahren Spendenbegünstigung ist jährlich von einem nachzuweisen. Abzugsfähig sind Spenden bis zu $0 \%$ des steuerlichen Gewinnes bzw. (bei Privatpe StG).

Aktuell sind 965 Einrichtungen spendenbe.

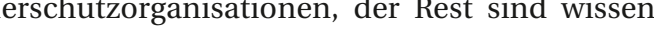

hitps://service.bmf.gvvat/service/allg/spenden/_start.asp
abgerufen am 19.05.2014 Dachverbände im Bereich des Behindertensports, (tur, sofern sie einen Osterreichbezug haben oder bestehen. Das Vorliegen der Voraussetzungen für irtschaftsprüfer zu bestätigen und der Behörde 


\section{BESONDERHEITEN IN DER PRAXIS}

\section{Iii) PRAXIS-INDIKATOREN.}

\section{Steuerbegünstigungen für CSOs sind vorgesehen}

Es gibt keine direkten oder indirekten (versteckten) Steuern

auf Zuschüsse/Subventionen/Zuwendungen.

. Steuervorteile für wirtschaftliche Aktivitäten von CSOs sind effektiv und unterstützen den Betrieb der CSO

genutzt und nicht sanktioniert.

4. (Gemeinnützige) Stiftungen können ohne große Verfahrens-

schwierigkeiten gegründet und ohne Verwaltungsaufwand

oder hohe finanzielle Kosten betrieben werden.

Absetzbarkeit von Spenden

6. Es gibt ein entsprechendes Verfahren, um Steuerbegüns-

tigungen für private und Unternehmensspenden zu erreichen.

lichem Interesse tätig sind, einschließlich von Mensche

recht- und Watchdog-Organisationen sind steuerlich

Nach Ansicht der befragten ExpertInnen liegen die häufigsten und größten steuerlichen Probleme b CSOs im Bereich der Umsatzsteuer und bei den Organisationen der bestehenden Risiken bewusst sind (Interview I3 16, 22). Fin Erklärungsversuch dafür lautet, dass in Österreich das unternehmersche Bewusstsein und das Wissen um Steuerpflichten im Zusammenhang mit dem Austausch von Leistungen gegen Entgelt generell unterentwicke sind. Deshalb würden gerade ideelle Vereine, die zunächst klein anfangen, aber allmählich wachse und ihre Tätigkeiten diversifizieren häufig in die $\mathrm{Ab}$ gabenpflicht kommen, ohne sich dessen gewahr z werden. Das gilt auch für die immer öfter anzutrefenden Tauschbörsen, Mitfahrgemeinschaften usw., bei denen es streng genommen ebenfalls z einem steuerbaren Leistungsaustausch kommt

Dazu kommt, dass die steuerlichen Fragestelungen bei CSOs, die häufig über stark diversifizier Einnahmequellen - Spenden, Subventionen, Förderungen, Erträge aus wirtschaftlichen Geschäftsbetrieben usw. - verfügen, überdurchschnittlich konplex sind und ein ausreichendes Maß an Rechtsscherheit nur mithilfe von SteuerexpertInnen hestellbar ist, die mit der Materie gut vertraut sind

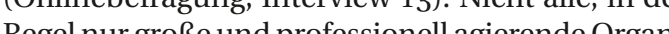
gen spruch und sind bereit oder in der Lage, die Kostedafür zu tragen (Interview I3 23). In der Previs kan es daher vorkommen, dass CSOs Steuerschulden Höhe von mehreren hunderttausend Euro anhëufen, die, sobald sie von der Behörde geltend gemach werden, die Organisation in ihrer Existenz bedrohen. Im Extremfall können sogar die Vorstandsmitglied in die persönliche Haftung genommen werden. gibt allerdings auch den umgekehrten Fall, das CSOs mangels Sachkenntnis mögliche Steuervo teile nicht in Anspruch nehmen (Interview I3, I6).
Dramatisch wird es für eine CSO vor allem dann, wenn die Behörde im Zuge einer Betriebsprüfung zu der Ansicht gelangt, dass die für eine Begünstigung erforderliche ausschließliche und unmittelbare Erfüllung des begünstigten Zwecks micht (mehr) gegeben ist. Tatsächlich scheint es zunehmend Verwaltungspraxis zu sein, entgeltliche Assistenzleistungen an andere CSOs, z. B. Personalgestellung, Überlassung von Infrastruktur, Buchhaltung usw., als begünstigungsschädlich zu qualifizieren (Onlinebefragung). In diesem Fall drohen der Verlust der Gemeinnútzigkeit und rückwirkende Abgabenforderungen, die dann nicht nur die Umsatzsteuer, sondern auch Ertragssteuern und Lohnabgaben (Kommunalsteuer) beinhalten können. Insbesondere die geforderte Unmittelbarkei der Zweckerfullung wid nach Meinung vieler ExpertInnen den heutigen Gegebenheiten, wo die Arbeitsteilung zugenommen hat und auch CSOs viefach zusammenarbeiten, nicht mehr gerecht (Interview
I3, I4, I6).

Es genügen aber schon Formfehler in der ding des begüntigten Zweckes nicht mit der tachung chen Geschäftsführung übereinstimmen um die steuerliche Beginntigung zu verlieren (Onlinebefragung, Interview I3).

In dem Fall wird die CSO bzw. deren steuerlicheVertretung das Finvernehmen mit der Behörde suchen und nicht selten enden derartige Verfahren mit einem ,Vergleich`, bei dem die CSO einen Teil der Steuerschuld begleicht, aber die steuerliche Begünstigung nicht verliert (Interview I3, 2I, 23). Manche kritisieren in dem Zusammenhang den Umstand, dass CSOs dadurch dem Wohlwollen der Beamten ausgeliefert sind, oder wie es einer der interviewten Steuerexperten drastisch formuliert: $: \mathrm{E}$ ner sitzt auf dem lângeren Ast und hat eine Sage in der Hand, um den Ast abzuschneiden (Interview I3). Andere wiederum sind der Meinung, dass die spielregeln ‘ bekannt seien und abgesehen von kle nen Nachschärfungen, etwa bei der steuerichen Behandlung von Vereinsfesten oder Beschäftgungsprojekten, in die herrschende Praxis nich gegriffen werden sollte (Interview I4).

Dass der Vorwurf der môglichen Abhängigkei vom Wohlwollen nicht völlig aus der Luft gegriffen ist, zeigt der Fall des Vereins gegen Tierfabriken (VGT), der nem $\$ 278$ auch eine Steuerprüfung über sich orghen lasen musste nachdem das BMF gegenüber den zusündusen Finnzantändes Vereins geäußert hatte ${ }^{85}$ In anderen F̈̈lle wenn es sich etwa um eine CSO im Nahebereich ner Gebietskörperschaft, politischen Partei oder ener anderen öffentlich-rechtlichen Einrichtun handelt, ist die Finanz aber auch einmal bereit rein Auge zuzudrücken und über offensichtlich begün http://www.ots.at/presseaussendung/OTS_20100223_-
OTSooio/tierschutzcausa-finanzant-bestaetigt-gemeinnuet- tigungsschädliche Umstände hinwegzusehen (Inerview I3, I6, 23).

Einen Graubereich gibt es auch bei der Kommunalsteuer, die von den Gemeinden eingehoben wird und deren Höhe zum Teil mit diesen rauszuhandeln ist (Onlinebefragung, Interview I6). Inzwischen gibt es außerdem Hinweise darauf, dass sich angesichts der prekären Finanzlage vieler Kommunützige, von der Kommunalsteuer befreite Betrieb in ihrem Gemeindegebiet anzusiedeln.

Die für die Beurteilung von steuerlichen Sach-

verhalten relevanten Vereinsich abien schaffen vielen Bereichen Klarheit, sind aber für die Behö den und Gerichte nicht verbindlich. Das erklär auch, warum nur selten Rechtsmittel gegen ersti stanzliche Entscheidungen ergriffen werden: die Erfahrung hat gezeigt, dass sich die Zweitinstanz der Unabhangige Finanzsenat, jetzt: Bundesfinan gericht - streng an die Gesetze hălt und die Entscheidung in der Regel zu Ungunsten der CSO austallt. Manche halten diesen Umstand unter den weil die Legislative hier der Exekutive enkin we dieren dafür beispielweise über den Weg von Verordnungen für mehr Klarheit Verbindlichkeit und Rechtssicherheit zu sorsen (Onlinebefragung Interview I3 I6, 22). Rechtsexperten warnen auch der Möglichkeit, dass in Zukunft häufiger erstins tanzliche Entscheidungen z. B. als Folge von Konkurrentenklagen durch das unabhängige Bundesfnanzgericht oder den VwGH aufgehoben und dam Präzedenzfälle geschaffen werden könnten, die weitreichende Folgen für alle gemeinnützigen $\mathrm{O}$ ganisationen haben (Interview 2I).

Inzwischen kann aber nicht ausgeschlosse bereich fur sich zu nutzen, indem sie beispielsweise (and Genuss eine steuetlichen Begünstigung fur eine gentlich gewerbliche Tatigkeit kommen. Insbesondere im Bereich der Beratungsdienstleistungen soll das immer wieder vorkommen (Interview 22). Die Finanzämter reagieren darauf mit vermehrten Ko trollen, was durch die Tatsache, dass Kapitalgeselschaften im Firmenbuch stehen, erleichtert wir Dennoch entsteht dem Sekto insgesamt ein Imageschaden, wenn Grenzen zwischen gewerblicher oder umgangen werden (Interview I3, I6, 22).
In Österreich gibt es, anders als in Deutschland, keinen Fest- oder Freistellungsbescheid übe exakte Auskunft darüber mögshalb auch kein meinnützige oder mildtätige Organisationen es in Österreich gibt. Manche CSOs haben sich in de Vergangenheit die Ordnungsgemäßheit ihrer Satzung von der für sie zuständigen Finanzbehörde formlos bestâtigen lassen. Diese Bestätigungen schützen die CSO allerdings nicht, wenn die Behörde im Zuge einer Betriebsprüfung zu der Ansich kommt, dass die CsO begunstigungsschädliche Aktivitaten gesetzt hat. Sie sind auch nur ein ungentigendes Mittel, um beispielsweise gegenúber aus landischen Behörden die steuerliche Begünstigun enderen keit zu kommen 政 für den darem inall immer ofter, indem sie schen Spenterin en Jour

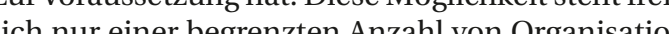
nen und nur für bestimmte gemeinnüzige bzw. mildtätige Zwecke zurVefügung So kommen twa Menschenrechtsorganisationen derzeit nur über den Umweg der Mildtätigkeit in den Genuss der Spendenabsetzbarkeit, während Tierschutzorganisationen, die keine Tierheime nach der Tierheimverordnung betreiben, von der Absetzbarkeit ausge schlossen sind. Auf Unverständnis stößt auch, das im -Kulturland Österreich Zuwendungen an Kunst(Interview I3, 21)

Bis 201 r wurden spendenbegünstigte Einrichders behandelt, als die (seit 2009) ebenfalls begünstigten 政 $\$ \$ 268 \mathrm{ff}$ des Unternehmensgesetzbuches entsprechenden Prufung durch einen beeideten Wirtschaltspiter unterziehen. Seit 2012 gilt diese Auflü die dungseinien Forschungs- und Erwachsenenbildeine Veriche na die Prufung besonders fur ist hat die Zahl dieser Einrichtungen seither deutlich abgenommen (FVA 20I3a).

86

ind die Akademie der bildenden Kinsten, Kunsthochschulen ungsfonds des Bundes und der Länder, ,iei Akademie der Wisrichtungen mit Sitz in einem Mitgliedstaat der Europäischen
Union oder einem Staat, mit dem eine umfassende Amtshilfe 


\section{VERBESSERUNGSPOTENZIALE}

Uber die folgenden Verbesserungsmöglichkeiten im ten SteuerexpertInnen und CSO VertreterInnen weitgehend Übereinstimmung:

\section{An die Adresse der Gesetzgebung:}

I. Die der Bundesabgabenordnung und anderen Gesetzen zugrunde liegenden und teilweise überholten normativen Konzepte der Gemeinnützigkeit und der Mildtatigkeit sollten breit zur Diskussion gestellt und im Lichte der Entwicklungen der letzten 5 Jahrzehnte im Sekto neu formuliert werden (Onlinebefragung Interview I4, I6, 22). Ziele einer solchen Reform sollten eine höhere Rechtssicherheit für CSOs, eine Vereinfachung der Verwaltungspraxis, der Abbau von bestehenden Diskriminierungen und die gezielte Förderung der Daseinsvorsorge und des zivilgesellschaftlichen Engagements in der Bevôlkerung bei gleichzeitiger Verhinderung von Missbrauch ielbest konnte auch in Fonm einer Staatsspielsweise für die Gleichstellung von Man und Frau, die Gleichbehandlung von Menscen mit Behinderungen und für den Um und Tierschutz bereits gibt. Das den CSOs eigene Prinzip der Ehrenamtlichkeit bzw. Freiwilligkeit - in Deutschland: Selbstlosigkeit sollte dabei eine besondere Würdigung erfahren (Interview 22). Tatsächlich hat die im Dezember 2013 angelobte Koalitionsregierung das Vorhaben in ihr Arbeitsprogramm aufgenommen, einen ,klaren Rechtsrahmen für gemeinnützige Organisationen ₹u schaffen, ohne dieses Vorhaben allerdings dort näher auszuführen. ${ }^{87}$

2. Die steuerliche Begünstigung von CSOs sollte mittels eines individuellen Rechtsaktes, z. B. durch einen Fest- oder Freistellungsbescheid und/oder die Eintragung in eine Liste ex ante festgestellt und ihnen dadurch schon bei de Gründung ausreichende Rechtssicherheit gegeben werden (Onlinebefragung, Interview 2I, 22, 23, 24). Um möglichen Missbrauch hin anzuhalten, wäre etwa eine Routineprüfung durch die Behörde oder in ihrem Auftrag späInterview 22).

. Die Forderung nach einer unmittelbaren we in Deuscill inderen, dienenden Organistionen weitergenen

werden dürfen (Onlinebefragung, Interview I3

I4).

auch von der geforderten ausschließlichen Zweckerfüllung abgerückt werden soll, um es dadurch insbesondere Privatstiftungen zu erlauben sich mehr als bisher gemeinnützig zu engagieren (Onlinebefragung, Interview I3).

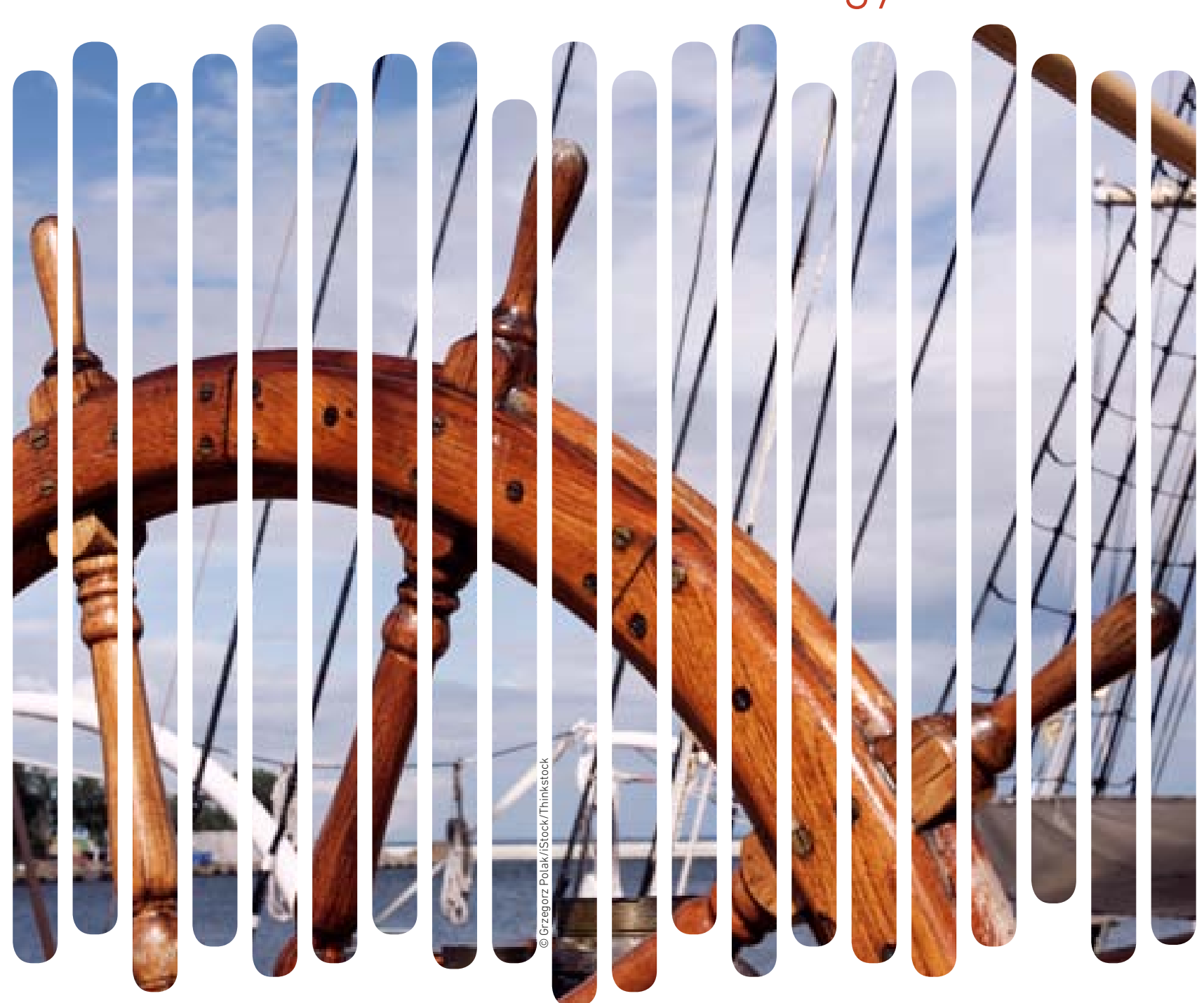

Dem Argument, dass dadurch die Kontrolle erschwert und dem Missbrauch Vorschub geleistet wird, halten ExpertInnen entgegen dass die heute zur Verfügung stehenden Methoden und Technologien der Rechnungslegung eine einfache und lückenlose Mittelve folgung möglich machen (Interview 13).

5. Die Regierung hat sich bereits vorgenommen in ausgewahlten Bereichen eine rechtliche Grundlage für gemeinnützige Stiftungen unter Einctictsur .

\section{8}

http:// www.bka.gv.at/DocView.axd?Cobld=53264 abgerufen
am 25.05.2014 Kostenersätzen geben, wie das heute bereits bei den Sportvereinen der Fall ist (Onlinebefra-
6. Für alle gemeinnützigen und mildtätigen CSOs sollte es die gleiche Moglichkeit der steuerund sozialversicherungsfreien Auszahlung von

gung). sammelnden Organisationen und Stiftungen, das zur Finanzierung der gemeinnützigen Tatigkeit verwendet wird, sollte so wie betriebWhes Vemong von der Kest-p

werden (Onlinebefragung).

Die Bereiche Gesundhecke, insbesondere die Jugend, Kunst und Kultur aure Beitet werden (Interview I3). Die Diskriminierung von Tierschutzorganisationen (ohne Tierheime) sollte beseitigt werden (Onlinebefragung).

9. Die maximalen Spendenabsetzbeträge sollten erhöht und auf mehrere Jahre verteilt werden können, so wie das heute schon in Deutschland der Fall ist, wo bis zu 20 \% des Jahresgewinns (bzw. Jahreseinkommens) oder bis zu 2 Mio. Euro, verteilt über Io Jahre abzugsfähig sind (Onlinebefragung). o. Die im nicht-unternehmerischen Bereich von CSOs anfallende Vorsteuer könnte durch eine Beihilfe z. B. in Form einer Investitionsprämie in Höhe der nicht abzugsfähigen Vorsteuer kompensiert werden, wie es sie bereits jetzt nach dem Gesundheits- und Sozialbereichgemeinnützige Rechtsträger gibt (Onlinebefragung).

\section{An die Adresse der CSOs und ihre}

Interessenvertretungen:

ce-Maßnahmen setzen und sich zu erhöhter Transparenz bei der Rechnungslegung - etwa in der Form der Spendengưtesiegelprüfung - ver-

plichten (Interview I3).
CSOs und ihre Interessenvertretungen sollten sich mit den Bestrebungen der Europäischen Union, die Gemeinnützigkeit und steuerliche
Begünstigungen für gemeinnützige Organisationen zu harmonisieren, auseinandersetzen onen beteiligen (Interview 22, 24)

3. Parallel dazu sollten alternative Modelle der steuerlichen Begünstigung von CSOs und der Abzugsfähigkeit durch Unternehmer und Privatpersonen, wie sie in anderen Mitgliedsländern, z. B. in Deutschland, UK, Ungarn und Rumänien existieren, geprüft und hinsichtlich ihrer Eignung für den gemeinnützigen Sektor gung).

$\overline{8}_{7}$ http: // www.bka.gv.at/DocView.axd?Cobld= 53264 abgerufen
am 25.05.2014

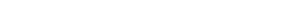




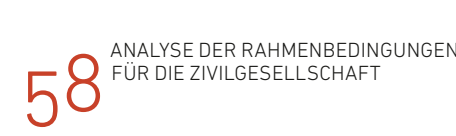

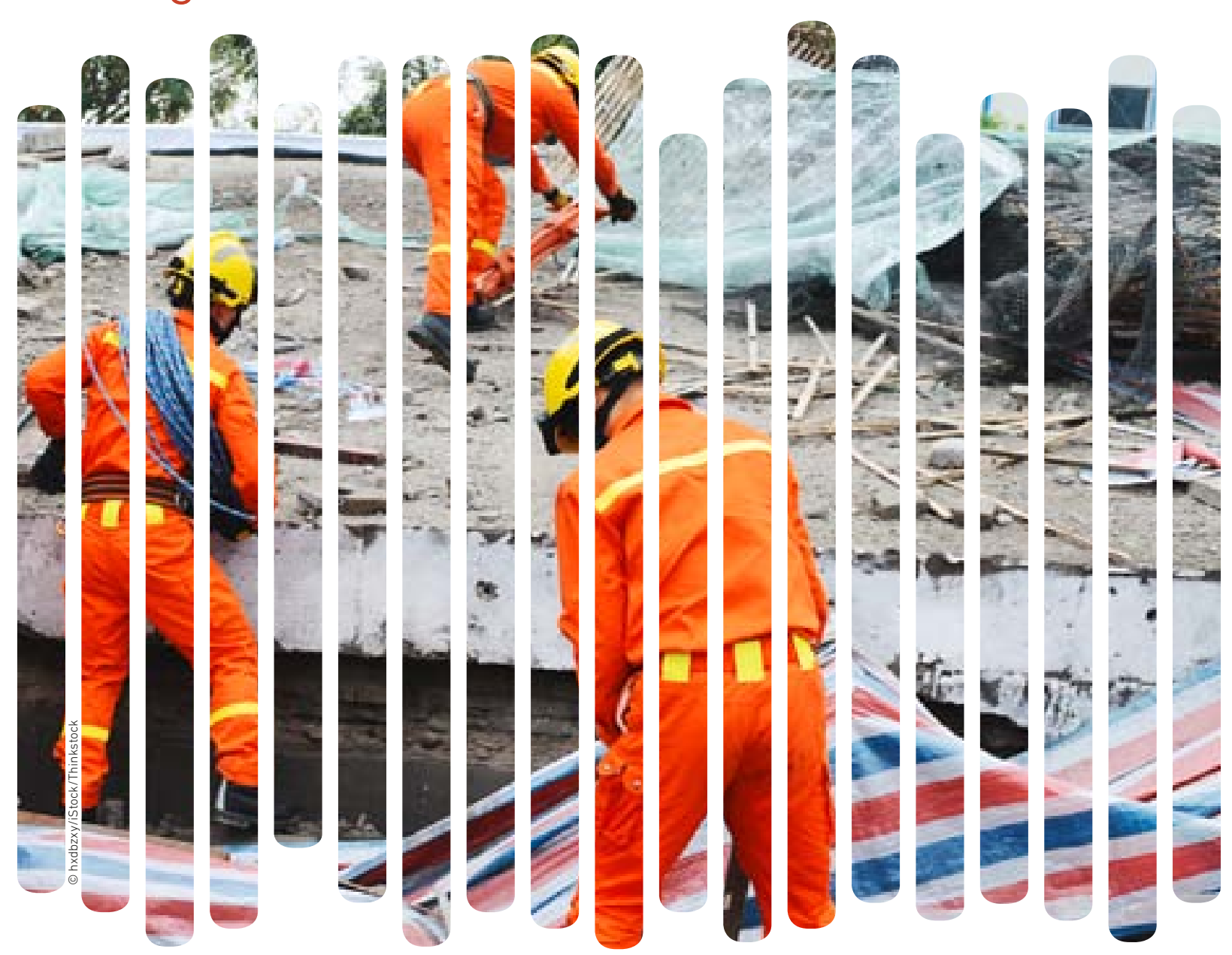

6. Beschäftigung und Freiwilligenarbeit

$D_{\text {ten zu einem wichtigen Beschäftigungsfeld }}^{\text {er Nonprofit Sektor ist in den letzen Jahrehn- }}$ avanciert (siehe auch Kapitel III/3). Eine Reihe von Entwicklungen läuft darauf hinaus, dass dieser Trend - zumindest für Teilbereiche des Sektors -
auch in Zukunft weiter anhalten wird. Fin Beispie auch in Zukunft weiter anhalten wird. Ein Beispiel
dafür ist der Pflegebereich, der vor allem durch die dafür ist der Pflegebereich, der vor allem durch die
demografische Entwicklung künftig noch an Bedemografische Entwicklung künftig noch an Be-
deutung gewinnen wird (Schneider et al. 2006). In deutung gewinnen wird (Schneider et al. 2006). In
Österreich spielt der Staat in Bezug auf die FinanOsterreich spielt der Staat in Bezug auf die Finan-
zierung von Pflegeleistungen traditionell eine wichzierung von Pflegeleistungen traditionell eine wich-
tige Rolle. Die letzten Jahre waren von der Frage getige Rolle. Die letzten Jahre waren von der Frage ge-
prägt, wie Pflegedienstleistungen künftig in prägt, wie Pflegedienstleistungen künftig in
ausreichendem Maße bereitgestellt werden können. Neben der Finanzierung der Pflegedienstleistungen gilt auch die Verfügbarkeit von (qualifiziertem) Pflegepersonal als besondere Herausforderung. Niedrige Löhne und schwierige Arbeitsbedingungen schmälern die Attraktivität dieses Berufsfelds. CSOs stehen bei Dienstleistungen im Sozial- und Gesundheitsbereich häufig vor der Herausforderung, den steigenden Bedarf an Leistungen bei stagnierenden oder rückläufigen Einnahmen von der öffentlichen Hand abzudecken (Simsa/More-Hollerweger 2013). Dies hat vielfach Folgen für die Arbeitsbedingungen und führt zu dem Paradoxon, dass CSOs, die sich in ihrer Mission sozialen Zielen ihre MitarbeiterInnen aufweisen und zur Prek

sierung der Beschäftigung beitragen (Dimmel 20I2). Gerade im Sozial-und Gesundheitsbereich gibt es ein überdurchschnittliches Ausmaß an prekären Beschäftigungsverhältnissen (Liebig/Karla 2003, Dathe et al. 2009), zudem ist Burnout in diesen Bereichen ein ernstzunehmendes Thema (Schober/Schober 2004). Das ist kein Spezifikum des Nonprofit Sektors, sondern vielmehr der jewei-

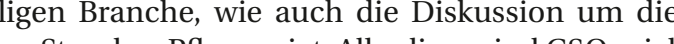
24-Stunden-Phege zeigt. Allerdings sind CSOs vielaltiv für die seselschatlichen Tatigkeltsbereichen wast Teben öbn als auch fur die vielen Freiwilligen zu. Z auch berit sind, auf vith or sich ehreatich zu engagien (Hader/Schneider 2009). Dies erklärt ach die vielen verschiedenen Beschäftigungsformen, die speziell m Nonprofit Sektor vorzufinden sind, wie Freiwilligenarbeit, Zivildienst, das Freiwillige Sozialjah und das Freiwillige Umweltschutzjahr etc. Wichtig sind daher entsprechende rechtliche Rahmenbedingungen, um zu gewährleisten, dass die Schnittstellen zwischen bezahlter und unbezahlter Arbeit gut und im Sinne der Beteiligten geregelt sind. Ziel gungen für CSOs und ihre Umsetzung in der Praxis darzustellen.
RECHTLICHE REGELUNGEN

\section{III) RECHTLICHE REGELUNGEN-INDIKATOREN}

Beschäftigung

geberlnnen behandelt.

Die Fordergeber sind verpflichtet, die im Rahmen der Kollek-

3. Es bestehen klare Regelungen zur Abgrenzung von Freiwilli$\frac{\text { genarbeit und Beschaftigungsverhaltnissen }}{4 \text {. Die Gesetzgebung fördert Freiwilligenarbeit, }}$

gute Absicherung und erlaubt gleichzeitig spontanes Freiwil-

ligenengagement.

5. Es gibt klar definierte rechtliche Bestimmungen für Vertrags-

verhattnisse und Schy

Es gibt klar definierte rechtliche Bestimmungen für Vertrags -

verhaltnisse und Schutzmaßnahmen fur Teilnehmerlnnen,
die ein freiwilliges soziales / ökologisches Jahr im In- oder

Ausland absolvieren.

CSOs werden von Gesetz und von der Politik weitgehend wie andere Arbeitergeber behandelt. Es gzw gemeinnützige Organisationen künftig in B zug auf die Arbeitskosten positiv zu diskriminieren und ihnen diesbezüglich Vorteile zu schaffen. Dies wird damit begründet, dass CSOs Überschüsse nicht an EigentümerInnen ausschütten sondern wieder für die Erfüllung ihrer Mission investieren und damit zu positiven gesellschaftlichen Wirkungen beitragen. Viele Bereiche des Nonprofit Sektors sind durch ihren Dienstleistungscharakter sehr personalintensiv. Österreich weist im Vergleich zum EU-Durchschnitt verhältnismäßig hohe Arbeitskosten auf. Der Abgabenkeil, d.h. die Summe aus Lohnsteuern, Sozialversicherungsbeiträgen un sonstigen lohnabhăngigen Abgaben der DienstgeberInnen und Dienstnehmerlnnen betrug im Jah 2013 in Österreich bei Einzelpersonen ohne Kinder rund $49, \mathrm{I} \%$ der gesamten Lohnkosten ${ }^{89}$. Vorschläge hinsichtlich einer positiven Diskriminierung vo gemeinnützigen NPOs zielen auf eine Reduzierung der Lohnnebenkosten. Bislang gibt es für gemeinnützige Vereine, die mildtätige bzw. gemeinnützige der-, Jugend-, Familien-, Kranken-, Behindertenlinden- und Altenbetreuung verfolgen, dahingeiir jene Dienstnehmer m wirtschaftichen Gescüftsbetrieb (unentedicher Hilfsbetrieb) oder in einem Gewingeresh des Vereins tätig sind $\left(\$ 872\right.$ KommStG I993 $33^{\circ 0}$ ). Keine Kommunalsteuer ist für DienstnehmerInnen im unm telbaren Vereinsbereich zu entrichten.

Nicht alle CSOs unterliegen einem Kollektivvertrag, für sie gelten demnach auch keine Tarifbestimmungen. Seit 2004 ist der BAGs Kollektivvertra für Organisationen in den Bereichen Gesundheit,

89

hittp: / / www.oecd-ilibrary.org/taxation/taxing-wages-2014-
tax wages-2014-en abgerufen am 26.04 2014

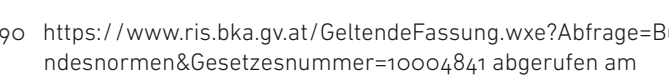
zu finanzieren, d. h. Förderungen zu valorisieren.
Soziales, Behindertenarbeit, Kinder-und Jugendwohlfahrt und arbeitsmarktpolitischen Dienstleistungen in Kraft der seit 2006 gesatzt und daher fiur alle Organisationen in diesen Bereichen gültig ist ${ }^{9}$. Was für die ArbeitnehmerInnen eine Verbesserun ihrer rechtlichen Absicherung ist, stellt CSOs dann vor Probleme, wenn im Rahmen des Kollektivvertrags beschlossene Gehaltserhöhungen durch Förderungen nicht abgedeckt werden, wie dies häufig der Fall ist. Dies bedeutet, dass eine Finanzierungslücke entsteht, die über andere Einnahmen abgedeckt werden muss oder - langerfristigzu einer Reduzierung des Personals führt (z. B. Interview 8).

Seit 2012 ist in Österreich das Freiwilligengesetz in Kraft. Demnach liegt ein freiwilliges Engagement vor, wenn natûrliche Personen freiwillige für geltlich, mit dem Zweck der Förderung der Allgemeinheit oder aus vorwiegend sozialen Motiven und ohne dass dies in Erwerbsabsicht, aufgrund eines Arbeitsverhalttnisses oder im Rahmen einer BeFreiwilligung engolgt, erbringen (\$ 2 Abs. 2 hal the weiters spezielle Regelungen fïr lige Souijo (im Intal), das Fillige Unlige Sozialjahr (im Inland), das Freiwillige Um land, bei denen es sich um längerfristige Formen (6-I2 Monate) des freiwilligen Engagements handelt und für die besondere Bestimmungen un Schutzbestimmungen gelten, beispielsweise hinsichtlich der Höhe eines Taschengelds und verschiedener Qualitätskriterien.

Trotz Bestehen des Freiwilligengesetzes sind die Grenzen zwischen Freiwilligenarbeit und E werbsarbeit vielfach nicht klar. Insbesondere be freiwilligen Einsätzen im Ausland besteht noch mangelnde Rechtssicherheit (Interview I0). Auch bei Menschen mit Lernbehinderung, die als nich arbeitsfahig eingestuft sind, ware es im Sinne eines Empowerments hilfreich, wenn sie im Rahmen de Tagesstruktur der Betreuungseinrichtung einfach - dann als Freiwilligentätigkeit eingestufte - Aufgaben erfüllen und in Folge auch entsprechend verschert und mit einem Taschengeld entlohnt werden könnten (Interview 4). Es gibt Kriterien, wie (a) keine vertragliche Verpflichtung zur Arbeitsleistung (b) kein Entgelt und (c) Wille zur freiwilligen Tätigkeit (Motivation), die Freiwilligenarbeit von Be schâftigungsverhältnissen abgrenzen. Ein BeschäfFreiwillighatnis unterscheidet sich von beitszeit Arbeitsort und Arbeitsablauf) und (ArlicheWeisungen. Es könnn zeiseitig und persönVereinbarungen getroffen werden, für die Freiwillige dann auch verantwortlich gem die Freiwi können (z. B. Schadensersatz bei unbegründeter Nichteinhaltung) (Interview 6 und Eder 2009).

http:/ / www.ris.bka.gvvat/GeltendeFassung.wxe?Abfrage=Bun

desnormen\&Gesetzesnummer $=20007753$ abgerufen am andere in einem organisatorischen Rahmen unentGedenkdienst, Friedens- und Sozioldienst im Aus-

http:// www.bags-kv.at/1001,35355,0,2.html abgerufen am 
Im Hinblick auf die Absicherung von Freiwil- ligenrats, das Freiwilligengesetz (2012), die Erstelligen bestehen große Unterschiede zwischen ver- lung von Curricula für Aus- und Weiterbildung sowie schiedenen Tätigkeiten. Freiwillige in Blaulichtor- von Instrumentarien zur Dokumentation der geleisganisationen (Rettung und Feuerwehr) sind gemäß teten Freiwilligenarbeit in Form eines Nachweises (S I76 Abs. I li.7a ASVG) bei Unfällen, die sie in Aus- über Freiwilligenarbeit und des Freiwilligenpasses übung freiwilliger Tätigkeiten erleiden, Arbeitsun- (Heimgartner 2009). Im Rahmen des Freiwilligengefällen gleichgestellt, das heißt sie haben Anspruch setzes ist auch die Erstellung eines Berichts über die auf Behandlung, Rehabilitation und Versehrten- Lage und die Entwicklung des freiwilligen Engagerente (Eder 2009) $)^{93}$. Für alle anderen Freiwilligen be- ments in Österreich in periodischen Abständen vo steht kein gesetzlich geregelter Versicherungs- gesehen ( $\$ 4$ Abs. I Freiwilligengesetz), der im Jah schutz, eventuelle Versicherungen (i.d.R. 20I4 das zweite Mal herausgegeben werden soll. Die Unfall- und Haftpflichtversicherung) werden teil- Berichte dienen der besseren Wahrnehmung von weise von den Freiwilligenorganisationen abge- Freiwilligenabeit in der Gesellschaft und als Grundschlossen. In denen letzten Jahren sind zunehmend lage für eine Evaluierung der von der Politik gesetzModelle entstanden, in denen seitens einzelner ten Maßnahmen. Der Osterreichische FreiwilligenBundesländer (z. B. Tirol, Vorarlberg) bei privaten rat, der sich aus VertreterInnen von FreiwilligenorVersicherungsträgern Haftplicht- und Unfallversi- ganisationen verschiedener gesellschaftlicher Berecherungen für Freiwillige abgeschlossen werden che, der Bundesministerien, der Landesregierungen (siehe auch folgender Abschnitt) (More-Hollerwe- der Sozialpartner sowie des Gemeinde- und Städteger/Hora 2014). Diskutiert wird immer wieder, in- bundes zusammensetzt, wirkt bei der inhaltlichen wieweit freiwilliges Engagement auch im Rahmen Ausrichtung des Berichts mit und hat auch weiter der Sozialversicherung (insbesondere Pensionsver- die Aufgabe das zuständige Bundesministerium für (Mordes Freiwilligenrates ist ebenfalls gesetzlich verandes Feiniligentios ist ebenfalls gesetzlich veran-

mindest einmal jährlich einberufen. Weiters bekennt sich der Gesetzeber zur Förderung des freiwilligen Engagements in Form von Zuschïses, die willigen Engagements in Form von Zuschüssen, di allerdings besteht kein Anspruch auf Förderungen (\$ 2 Freiwilligengesetz).

Es gibt wenige Förderprogramme, die Freiwilligenorganisationen gezielt bei der Gewinnung

2. Es gibt Anreize und staatlich unterstützte Programme für die Entwicklung und Förderung der Freiwilligenarbeit.

3. Anreize und Programme sind transparent und für Organisationen der Zivilgesellschaft leicht erhältlich. Politische Maßnahmen, strategische Dokumente oder Gesetze werden vollständig umgesetzt, überwacht und in regelmäßigen

Abstanden in partizipativer Weise evaluiert. Falle von

Als problematisch wurde im Rahmen der Interviews erwähnt, dass sich durch die zunehmende Auslagerung von sozialen und gesundheitlichen Dienstleistungen von der offentlichen Hand in den Nonprofit Sektor oft auch die Arbeitsbedingungen für die Beschaftigten verschlechtern. Tellweise schwierig ist

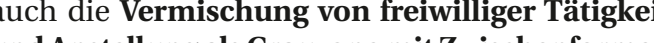

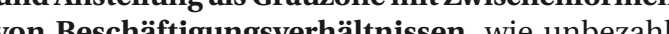
Üscher jekte mit Langzeitarbeitslosen mit keiner Arbeitsverpflichtung und keinem Entgeltanspruch, $z$ B. Beschäftigung von Arbeitslosen zur Reintegration in sozialökonomischen Betrieben (SÖB) über AMS (Interview 6).

In Österreich war das von den Vereinten Nationen ausgerufene internationale Jahr der Freiwillgen 200I ein wichtiger Impuls für die Bundespo tik, sich mit Freiwilligenarbeit auseinanderzusetzen. Seither wurde eine Reihe von Maßnahmen gesetzt um das freiwillige Engagement in Österreich zu fördern, dazu zählen die Etablierung eines Freiwil-

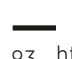

http:// www.ris.bka.gv.at/GeltendeFassung. wxe? Abfrage $=B$
desnormen\&Gesetzesnummer=10008147 abgerufen am und Betreuung ihrer Freiwilligen unterstützen. In

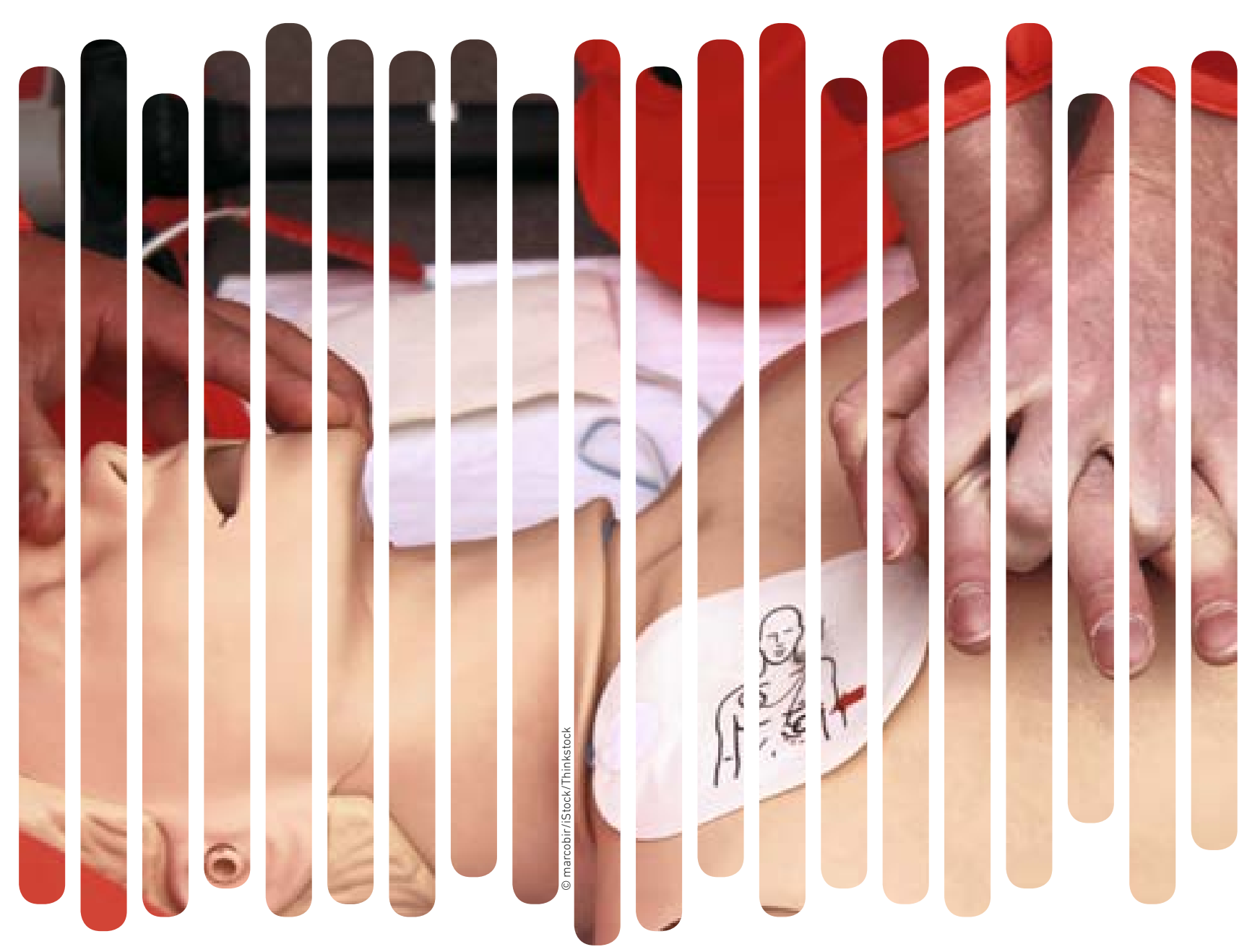

Als weiteres Thema wurden positive Anreize

einer Erhebung von rund 700 Freiwilligenorganisationen wurde eine offene Frage zu Verbesserungen der Rahmenbedingungen gestellt (More-Hollerweger/Hora 2014). Besonders häufig wurde erwähnt dass eine Unterstützung in Bezug auf die Unfall$60 \%$ der Organisationen sind Freiwillige durch ein Haftpflichtversicherung geschützt, wobei $49 \%$ der Organisationen selbst dafür aufkommen und be II \% der Organisationen andere Institutionen die Haftpflichtversicherung der Freiwilligen uberne men. Bei der Unfallversicherung ist der Anteil der Geschüzten etwas geringer. Hier sind $43 \%$ durc die Freiwilligenorganisation selbst und I3\% durch eine andere Institution geschützt. Eine Auswertung nach Bundesländern zeigt, dass offenbar einige O ganisationen nicht über bundeslandweite Versich ungen, wie sie in Vorarlberg und Tirol gegeben sin Bescheid wissen (More-Hollerweger/Hora 2014) Bencher lende Rechtssicherheit bei Aufwandsentschä gungen, einge Organisationen winchen sich hier ven (More-Hollerweger/Hora 20I4). Aufwandsentschädigungen und pauschalierter Aufwandsersatz kö bei Freiwilligenarbeit als verstecktes Gehalt ausgelegt werden (Interview 6). Großzügige Ausnahmen gibt es hier nur bei den Sportvereinen. Oft gefordert wurde auch die Anrechnung von freiwill cherung (More-Hollerweger/Hora 2014), worübe allerdings keine Einigkeit besteht. GegnerInnen gumentieren, dass esetzesnummer=10008147 abgerufen am 


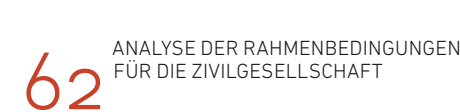

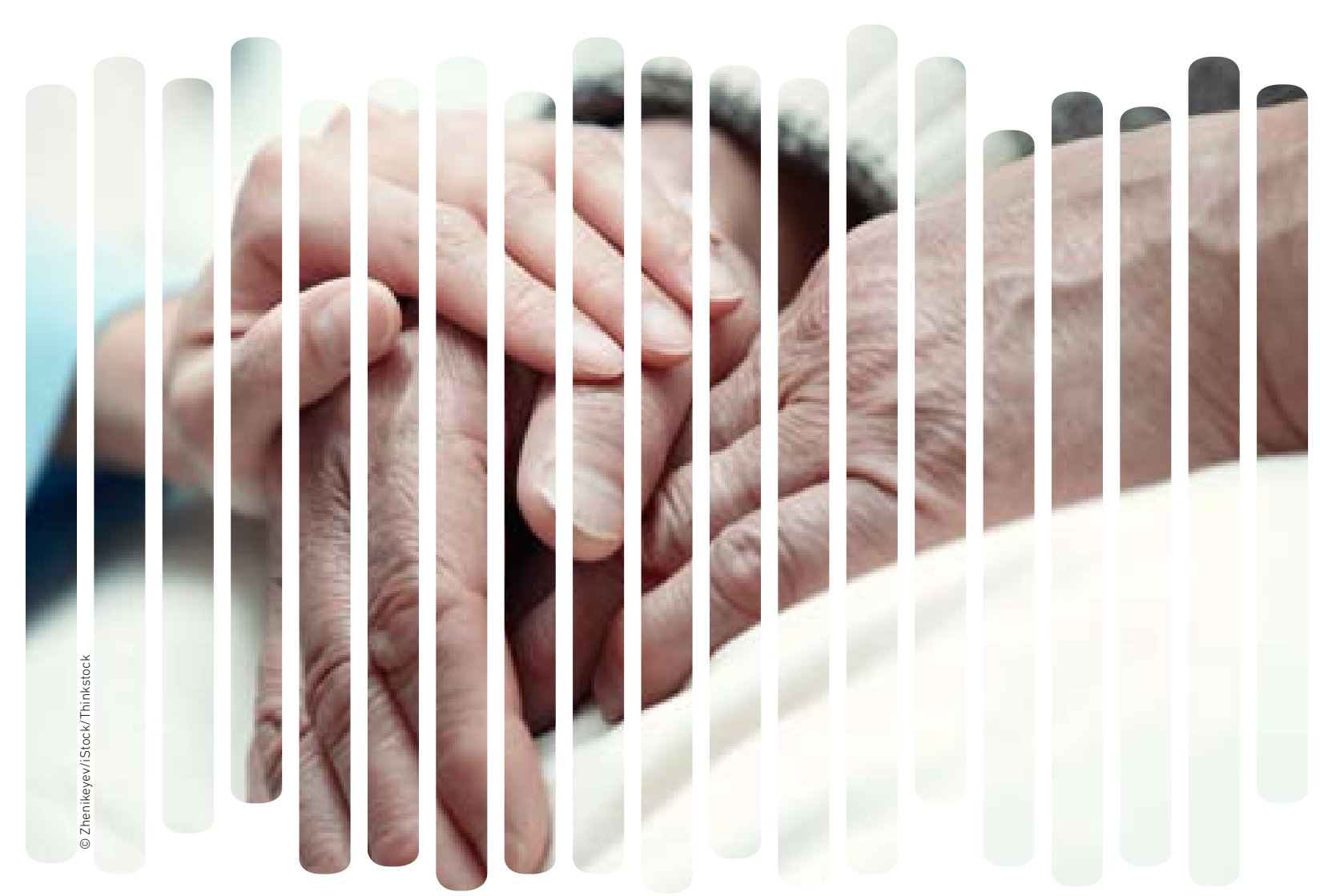

7. Bereitstellung von Dienstleistungen (Leistungsverträge/Förderungen)

$C_{\text {wobei der Staat als wichtiger Auftrastungen an, }}^{\text {SOnd bör }}$ dergeber fungiert und damit die Aktivtäten von CSOs in vieler Hinsicht beeinflusst. Im Folgenden werden der rechtliche Rahmen und die praktische Ausgestaltung der Beziehung zwischen CSOs und Dienstleistungen analysiert.

RECHTLICHE REGELUNGEN

W. RECHTLICHE REGELUNGEN-INDIKATOREN

\section{CSOs als Bereitsteller von Dienstleistungen}

Die bestehende Gesetzgebung ermöglicht CSOs Leistungen

in verschiedenen Bereichen, wie Bildung, Gesundheit, soziale Dienste anzubiete

Belastungen für CSOs vor, die nicht auch für andere Dienstleister existieren

Staat als Nachfrager sozialer Dienstleistungen

von Dienstleistungen vor, die von CSOs erbracht wenen Arten

könnten, einschlienlich mehrï̈hriger Mittel.

4. Es gibt keine rechtlichen Beschränkungen für CSOs, öffent-

liche Mittel für die Bereitstellung verschiedener Dienste zu

erhalten (entweder durch Leistungsvertrag oder durch

5. CSOs können langfristige Verträge für die Erbringung von

Verfügbarkeit von öffentlichen Mitteln für CSOs

6. Es gibt Gesetze oder andere Formen von Regelungen, die die staatliche Förderung für die institutionelle Entwicklung von
$\mathrm{CSOs}$, Projektförderungen und Co-Finanzierung von EU-Pro-

Es gibt auf nationaler Ebene einen Mechanismus für die Ver-

teilung der öffentlichen Mittel an CSOs
In Österreich sind CSOs in vielen gesellschaftlichen Bereichen, wie Bildung, Gesundheit, soziale Dienste, Kunst/Kultur, Sport etc. tätig. Dabei stehen sie häufig in unmittelbarer Konkurrenz zu anderen (offentlichen und gewinnorientierten) Anbietern wie das beispielsweise bei Schulen, Altenheimen, Krankenhäusern und Kindergärten der Fall ist, wo de verschiedenen Träger sehr ähnliche Dienstleistungen anbieten. Vielfach ist der Charakter der so sind Angebote der Sportvereine in der Regel partizipativer ausgerichtet, als beispielsweise jene von Fitnesscentern. Im Bereich der Bildung unterscheielle Ausrichtung, in Osterreich sind das besonder konfessionelle Anbieter, wie die katholische ode evangelische Kirche, jedoch auch Träger, die spezielle padagogische Konzepte verfolgen (z. B. Montessori oder Waldorfpädagogik). Grundsätzlich ist es gen aufzutreten, allerdings gibt es die Problematik, keit und die Beteiligung an Ausscheibunizigren im Widerspruch stehen kösch da gemanGemeinnützigkeit und damit verbund dice Bün tigungen ausschlaggebend sind, nicht mehr erfültigungen ausschlaggebend sind, nicht mehr erfülnützigkeit zu verlieren Dienstleistung im Nonprofit Bereich ein andererdet sich das Angebot oft durch eine bestimmte idefur CSOs möglich, als Anbieter von Dienstleistunzige CSOs Gefahr laufen, die Kriterien, die für die

\section{3}

Der Staat ist ein wichtiger Auftraggeber bzw. Funktionen und alternative LeistungserstellungsFörderer verschiedener Dienstleistungen. Wie be- prozesse, die einen gesellschaftlichen Mehrwert erreits in Kapitel II/3 erläutert, stammen rund 53\% zeugen, erschwert wird. Daher wird nicht immer der Einnahmen von CSOs in irgendeiner Form von die beste Qualität erreicht (Interview 8). Forder öffentlichen Hand (Schneider/Haider 2009). Grundsätzlich gibt es zwei verschiedene Formen, wie der Staat als Nachfrager von Dienstleistungen auftritt (Anastasiadis et al. 2003):

. Leistungsverträge 94 : hier werden klar definierte Leistungen beauftragt. Rechtliche Grundlage dafür ist das Vergaberecht.

. Förderungen ${ }^{95}$ : Fordergegenstände können Projekte, Infrastrukturkosten etc. sein. Der Detaillierungsgrad dessen, was gefördert wird variiert stark. Es kann zwischen Förderungen durch Bescheid (im Rahmen der Hoheitsverwaltung) und Fordervertragen (im Rahmen de Privatwirtschaftsverwaltung) unterschieden werden. Rechtliche Grundlage dafür sind das allgemeine Verwaltungsrecht, das öffentliche
Haushaltsrecht, das Zivilrecht sowie EU-FörHaushaltsrech
derrichtlinien.

derrichtlinien

CSOs treten sowohl als Auftrag- als auch als jührige Verträgu (nterview 2 . auch häufig einiuhre Verträge, die verlängert werden (Interview 3 ). Seitens der EU geht derTrend weg von sehr langfristigen Verträgen, um den Wettbewerb anzukurbeln (Interview 9 ).

In den letzten Jahrzehnten tendiert die öffentliche Hand dazu, vermehrt Leistungsverträge zu vergeben, um im Rahmen von Vergabeverfahren denWettbewerb zu stärken. Dies wird vor allem von der EU-Wettbewerbspolitik forciert. Auch im Rahmen von Förderverträgen werden die Leistungen zunehmend enger definiert und kaum noch Subventionen, die relativ frei verfügbar sind, vergeben Entscheidenden Einfluss haben hier wiederum europaische Rahmenbedingungen. Das Beihilfenrecht (Art 87 EG) legt fest, welche finanziellen Vorteile Behörden eines Mitgliedsstaates Organisationen, die Leistungen der Daseinsvorsorge erbringen, gewähren dürfen. Beihilfen, wie sie Subventionen darstellen, müssen von der Beihilfenaufsich der Kommission nostrifiziert werden. Förderungen, die für die Erbringung einer konkreten Leistung gewährt werden, zählen hingegen nicht als Beihilfe (Neumayr 20I0).

Positiv wird vermerkt, dass CSOs durch die Entwicklung weg von Subventionen hin zu Leistungsverträgen von Bittstellern zu gleichberechtigen Partnern avancieren (Neumayr 20I0). So dürfen beispielsweise vergaberechtlich Festpreise für max (206) sungen vorgenommen werden (Interview 9). Bei Förderungen gibt es häufig keine entsprechenden Indexklauseln (Interview 8), wie weiter unten noch ausführlicher erläutert wird. Die Betonung des Leistungsvertragscharakters birgt allerdings auch Nachteile in sich. KritikerInnen argumentieren, dass durch die Ausschreibungsverfahren der Preis in den Vordergrund rückt und der Spielraum für andere chungsarbeiten ${ }^{96}$ untersuchen, inwieweit Leisungsverträge bzw. klar spezifizierte Förderverträge Funktionen der Interessensvertretung bzw. der Gemeinschaftsbildung, die CSOs vielfach ubernehmen, verdrängen. Argumentiert wird, dass die Querfinanzierung dieser Funktionen nicht meh möglich ist bzw. die wachsende Professionalisieung zu Verdrăngungseffekten führt (Neumay ore. Ein Beispiel dafur ist der Einsatz von Freiwiligen. In Osterreich ist etwa die Erbringung von Dienstleistungen im Feuerwehr- und Rettungswesen bislang untrennbar mit einem ausgeprägten reiwilligenwesen verbunden. Rettung und Feuerwehren erbringen aber nicht nur die entsprechenden Dienstleistungen, sondern erfüllen eine Reihe von anderen wichtigen Funktionen (Schober et al. ÖI2). Alleine die Feuerwehr zăhlte im Jahr 2012 in Osterreich 338.664 Feuerwehrmitglieder ${ }^{97}$ (knapp (1) das heißt coln

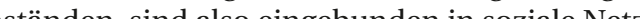
erke Aut sich für die freiwilligen MitarbeiteInnen im Rettungswesen. Das heißt CSOs erzieen Wirkungen, die weit über die Erbringung der unmittelbaren Dienstleistung hinausgehen. Insofern ist es problematisch, wenn gewinnorientierte Organisationen, die kein Interesse an diesen zusätzlichen Wirkungen haben und Überschüsse an ihre EigentümerInnen ausschütten, in Konkurrenz mit CSOs stehen, weil sie im Rahmen der Ausschreibung ausschließlich an der Erbringung der spezifischen Dienstleistung gemessen und diese zusätzlichen Wirkungen nicht berücksichtigt werden. Problematisch ist es auch, wenn einzelne Diensteistungen (z. B. Krankentransport) aus einem Verund mehrerer Dienstleistungen (Krankentransport und Rettungsdienst) herausgegriffen und usgeschrieben werden. Einerseits gehen Synergieffekte verloren, andererseits bedienen gewinnorientierte Unternehmen eher jene Felder, die gewinnbringend geführt werden können, währen CSOs ihrem ideellen Zweck entsprechend den Blick auf das Ganze bewahren und es hier auch zu eine Querfinanzierung zwischen Bereichen kommt. (Q) mehr und es werden zusätzliche zittel eror itstellung bestimmter Dienstleistungen birg ench

97 http://Www.bundesfeuerwehrverband.at/fileadmin/user_-
upload/Downloads/Statistiken/Statistik_2012.pdf abgerufuen

Der Begriff Leistungsvertrag ist nicht eindeutig definiert Neumayr (2010) bezeichnet beispielsweise Förderungen $\mathrm{m}$
hohem Detaillierungsgrad ebenfalls als Leistungsverträge

95 Auch der Begriff förderung ist nicht eindeutig definiert bzw und Subventionen (Neumayr 2010). 


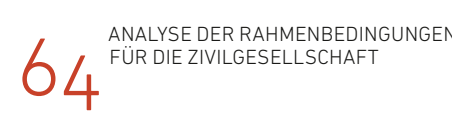

brochen und partizipative Elemente in der Leisungserbringung erodiert werden und letztlich auch ökonomische Nachteile daraus resultieren (Interview 2). Neue Ideen, die Vielfalt von Anbietern und Qualität gehen verloren, wenn die Erbringer von definierten Dienstleistungen nach dem Billigstbieterprinzip eruiert werden

Es gibt daher Bestrebungen, das Kriterium der Gemeinnützigkeit im Vergaberecht (stärker) zu ver-
ankern. Für den Rettungsdienst ist dies auf EUEbene bereits gelungen. In der Konzessions- und der Vergaberichtlinie wurde ein Gemeinnützigkeitsvorbehalt auf europaischer Ebene aufgenommen. Dies bedeutet, dass Träger des Rettungsdiensts (z. B. Gemeinden) diesen ohne formale europaweite Ausschreibung an gemeinnützige Organisationen übertragen können (Glanzer/Dempfer 201498). Be gründet wird dies damit, dass der Charakter ge meinnütziger Organisationen oder Vereinigungen (ohne Erwerbszweck) nur schwer gewahrt werden könnte, wenn die Dienstleistungserbringer nach den in dieser Richtlinie festgelegten Verfahren ausgabe- und Konzessionsrichtlinie in österreichisches Recht wird für Frühjahr 2016 erwartet.

Förderungen durch die öffentliche Hand sind, rorderungen durch die offentiche Hand sind, terschiedlich geregelt. Figene Regelungen, sehr unplizit die Förderungen von CSOs betreffen, gibtes plizit die Forderungen von CSOs betreffen, gibt es gendwo verankert ist Dennoch sind beispielsweise Vereine als förderbare Institutionen oftmals explizit genannt und CSOs faktisch Nutznießer von Förderungen in vielen gesellschaftlichen Bereichen, wie im Bereich Kunst und Kultur, Sport, soziale Dienste etc. Nationale öffentliche Mittel an CSOs sind berücksichtigt in den Budgets von Bund, Land und Gemeinden. Die Höhe der Mittel ist oft nicht rechtlich geregelt, sondern liegt im Ermessen von Politik und Verwaltung. Mangels statistischer Erfassung kann der Anteil der Förderungen für CSOs in Österreich nicht ermittelt werden.

http://www.roteskreuz.at/ berichten/news/datum/2014/
$04 / 1 / 1 /$ gastkommentar-ist-die-rettung-gerettet/ abgerufe

$0411 / 1 /$ astktkom
am 26.04 .2014

99 http://www.forum-vergabe.de/fileadmin/user_upload/

\section{GESONDERHEITEN IN DER PRAXIS}

-INDIKATOREN

CSOs als Bereitsteller von Dienstleistungen

CSOs sind in der Lage, Verträge im Wettbewerb mit anderen

tungsbereichen (z. B. Bildung, Gesundheit und Forschung)

von Dienstleistungen (Bedarfsanalyse, Bestimmung der

Dienstleistungen, die den Bedürfnissen am besten entspre-

Staat als Nachfrager sozialer Dienstleistungen

CSOs erhalten ausreichende Finanzierung, um die Grund-

kosten für die Dienstleistungen, die sie vertraglich vereinbart

haben, einschließtich der anteiligen institutionellen (Over-

\section{Verïg}

Ver

ohne drastische Vermin-

g von Jahr zu Jahr und die Höhe des Budgets für CSOs

Die Teilnahme von CSOs am öffentlichen Förderzyklus ist

CSOs bieten in Österreich ein breites Spektrum an erschiedenen Dienstleistungen in vielen gesellschaftlichen Bereichen an. CSOs beteiligen sich an allem zwei Beispiele von Ausschreibungen in Österreich, an denen sich CSOs und gewinnorientierte Unternehmen in Konkurrenz beteiligten und die medial sehr stark diskutiert wurden

\section{Flüchtlingsbetreuung - Betrieb der Bundes-}

betreuungseinrichtungen Traiskirchen,

Thalham, Bad Kreuzen und Reichenau: Im

Jahr 2003 fiel die Entscheidung bei der Aus-

schreibung über die Ausgliederung der Bun-

desbetreuungs-Einrichtungen zwischen den

Finalkandidaten ,European Homecares und

einem Konsortium von Rotem Kreuz, Caritas,

Diakonie und Volkshilfe. Die Entscheidung gin

zugunsten der deutschen Firma ,European

Homecare aus. Diese hat den Vertrag im Jahr

2010 wegen mangelnder Rentabilität gekündig.

hat die Schweizer Firma ORS im Jahr 2012

erhalten.

$\rightarrow$ Rettungsdienst Tirol: Das Land Tirol hat in

Jahro

Rettungsdienst vergeben. Neben dem Tirole

Bieterkonsortium bestehend au Rotem Krer

Arbeiter-Samariterbund, Johanniter-Unfall-

Hilfe, Malteser Hospitaldienst und Österreichi-

schem Rettungsdienst hat sich die dänische

Firma Falck beworben, letztere hat den

Zuschlag letztlich aber nicht erhalten. Das Bie-

terkonsortium musste den Preis nachbessern,

was eine Klage der Firma Falck zur Folge hatte.

Diese wurde in erster Instanz abgewiesen und

liegt derzeit beim Verwaltungsgerichtshof.

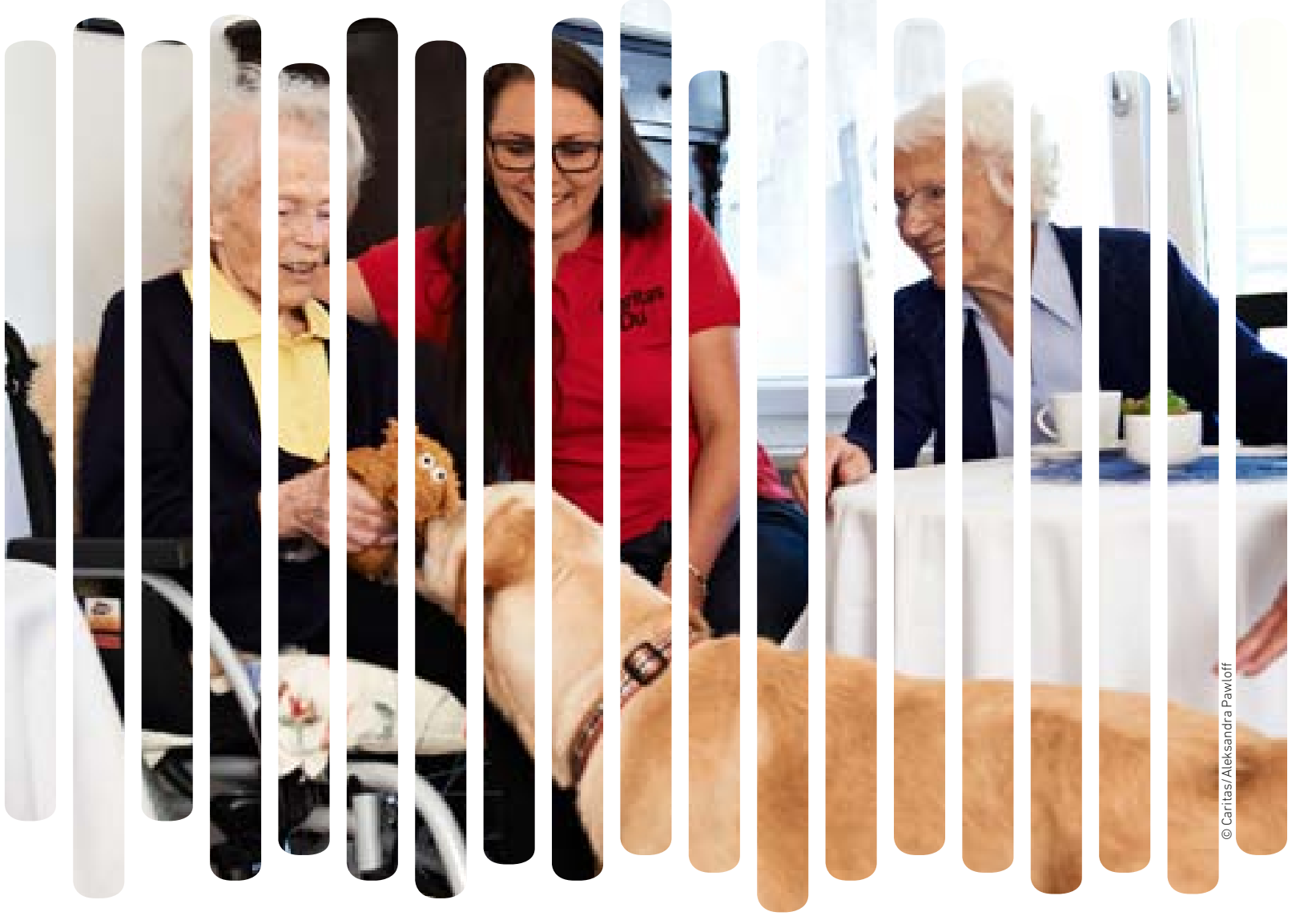

Die Kritik im Rahmen dieser Ausschreibun- tende Rolle. Eine Vielzahl an Leistungen wird daher gen richtete sich einerseits gegen die gewinnorien- im Rahmen von Förderverträgen erbracht. Die Förtierten Unternehmen. Der Vorwurf lautete, dass derbedingungen sind je nach Tätigkeitsbereich diese bei der Qualität Abstriche machen würden sehr unterschiedlich, teilweise sind mehrere Ge(Falter ${ }^{100}$ I2.07.20I I). Zudem wurde das Vorgehen bietskörperschaften an der Finanzierung bestimmder öffentlichen Hand kritisiert. Im Falle der ter Dienstleistungen beteiligt. Vielfach ist der SparFlüchtlingsbetreuungen wurde ihr vorgeworfen, kurs der letzten Jahre deutlich spürbar, das trifft dass sie bewusst das gewinnorientierte Unterneh- aber nicht auf alle Organisationen zu. Beispiels-
men bevorzugt hätte, weil dieses kein politisches
weise hat die Bundes-Sportförderung in den letzten Interesse hătte und daher ein rangenehmerer Ver- Jahren kontinuierlich zugenommen. In einer Erhetragspartner sei (ebd.). VertreterInnen von CSOs ha- bung von 53 CSOs war die finanzielle Situation der ben erwähnt, dass es sehr schwierig sei, Informati- Jahre 2008-2012 im Sample überraschend stabil onen von den Anbietern zu erhalten, weil diese (Simsa/More-Hollerweger 2013). In der Summe ist offenbar im Rahmen des Vertrags zur weitreichen- sie für die befragten Organisationen gleich geblieden Verschwiegenheit verpflichtet wurden und da- ben. Je $6 \%$ geben an, dass sich ihre finanzielle Situher tur ene Advocacy-Arbeit wichtige Informatio- ation stark verbessert bzw. stark verschlechtert hat, nen nicht weitergeben dürfen (Interview 5). Im 34\% haben Verbesserungen und 30\% haben VerFalle der Rettungsienste wurde der offentichen schlechterungen erlebt, fur $24 \%$ hat sich die Situawust eins Tusst ensetze, un die Predse Auld dicken (Tinor

Im Rahmen der Interviews wurde angemerkt, den letzten vier Jhren deutlich getiegen $96 \%$ be

Im Rahmen der Interviews wurde angemerkt, den letzten vier Jahren deutlich gestiegen. $96 \%$ besich an Ausschreibungen zu beteiligen, weil dafür mehr a $20 \%$. Fin Großteil der NPOs (80\%) hat das ein großes juristisches Know-How erforderlich sei quantitative Angebot gesteigert, $27 \%$ sogar um und die Beteiligungen an Ausschreibungen sehr ar- mehr als 20\%. Über 80\% bieten eine größereVielfalt beitsintensiv seien (Interview II). In vielen Bereichen in denen CSOs aktiv sind, keiten, Leistungen für neue Zielgruppen, neue Inspielen Förderungen nach wie vor eine bedeu- halte oder Vernetzungsarbeit (ebd.). Gerade im So一

htp://www.falter.at/falter/2011/07/12/profit-mit-not/abge-

hitp:///www.t.com/home/5612768-91/rettungswesen-aus-
schreibung-war-laut-ex-rk-leiter-ein-platter-diktat.
csp?tab=article vom 25.10 .2012 abgerufen am 14.04 .2014 halte oder Vernetzungsarbeit (ebd.). Gerade im Soaufgrund der demografischen Entwicklung massive zusätzliche Mittel erforderlich sein (Interview 8). Im Rahmen einer weiteren Erhebung (IGO ach der Zufriedenheit mit den Förderbedingun- 
gen gefragt. $62,2 \%$ der befragten Organisationen, Liquiditätsproblemen, die vor allem für kleinere die Fördervereinbarungen mit der öffentlichen Hand haben, gaben an, dass sie mit den bestehenden Regelungen nicht zufrieden sind. Als dringlichstes Problem wurde die mangelnde Valorisierung der Leistungen genannt. Knapp $46 \%$ nannte dest drei Jahren) als wünschenswerte Verbesserun (Interview 8). Generell haben vor allem klein sind ganisationen Probleme, Risiken einzugehen auße sie sind hoch spezialisiert (Interview II). Mitunter ist die Nachfrage nach den Dienstleistungen durch die CSOs nicht beeinflussbar, sondern wird durch die öffentliche Hand gesteuert, beispielsweise be Zuweisung von AsylwerberInnen. Die Folge diese Entwicklungen sind ein sukzessiver Konzentrationsprozess und oft ein hohes Maß an Selbstausbeutung seitens der CSOs (Interview 5 und II).

\section{- Einmischung in Details der Leistungserbringung} durch öffentliche Hand; Bürokratie bei der Abrechnung schung der öffentlichen Hand bzw. ihrer nachgeordneten Dienststellen in Details der Leistungserbringung angesprochen. Gemessen wird oft nich die Qualität der Leistungserbringung, sondern es wird kontrolliert, was an Input einfließt. Dies zeig sich insbesondere bei der Abrechnung, die sehr buMitunter wird erst im Nachhinein festgandegt, itur mit auch bestimmt wo förderbar sind (Interview 4). Fin Interviewartne

Quelle: IGO (2013)

In Bezug auf die Förderbedingungen wurde uch im Rahmen der Interviews eine Reihe von Problemen aufgezeigt:

\section{- ökonomische Risiken}

Die bereits oben genannte mangelnde Indexanpassung (Valorisierung) von (mehrjährigen) Förderungen wurde im Rahmen der Interviews ebenfalls bestätigt. Oft entstehen Finanzierungslücken, wen beispielsweise kollektivvertraglich vereinbarte $\mathrm{Ge}-$ haltserhöhungen stagnierenden Forderungen gegenüber stehen. Problematisch stelt sich fur vie Organisationen auch die Tatsache dar, dass zwar die Grundkosten für Dienstleistungen, die Overhea kosten jedoch nicht oder nur unzureichend gedeckt sind (Interview 4). Mitunter können CSO gesetzlich vorgeschriebene Auflagen (z. B. fregestellter Betriebsrat bei bestimmter Betriebsgröße nicht erfullen, weil diese vom Fördergeber nicht ab gedeckt werden - d. h. es müssten dafur alternative Finanzierungswege gefunden werden, was für ma che CSOs schwierig ist. Die Forderbedingungen . iko noch erhöht (Interview 4). Thrifent n fallo erfolgte die Unterzeichnung von Förderverträgen für das laufende Jahr erst Ende Dezember die Auszahlung dann noch später D. h. das Risiko liegt bden CSOs. Zwar handelt es sich dabei um eine Auftragserteilung durch konkludentes Handeln dings müssten CSOs bei einer Klage gegen die lehnung der Förderung das Prozessrisiko tre Außerdem besteht bei mehrfachen Abhängigkeit verhältnissen das Risiko, andere Projekte zu verlieren, wenn die CSO vor Gericht geht (Interview II) Verzögerungen bei Auszahlungen von öffentlichen
Mitteln an CSOs führen häufig zu ernsthaften bezeichnete dies als Ersatzhandlung - es werde geund relevant ist (Interview $\mathrm{I}$ ). sowohl für die öffentliche Hand als auch für die CSOs ein sehr hoherVerwaltungsaufwand. Problematisch ist hier weiters, dass verschiedene Fördegeber unterschiedliche Abrechnungssysteme haben, die nicht kompatibel sind, sodass der Aufwand bei der Abrechnung und damit Overhead-Kosten nochmals erhöht werden (Interview 4, 8, II).

Problematik: Konflikt Advocacy-Funktion

$\leftrightarrow$ Abhängigkeit von ơffentlicher Hand

Durch die hohe Abhängigkeit von der offentlichen Hand in vielen Sektoren können CSOs nicht die die Gefahr der Vereinnahmung durch den Staat (Interview 5, 11). CSOs haben in vielen Bereichen eine gen ihrer Leistungsemptangerlnnen kennen und verhante one Wahichung. Dadurch fehlt eine intensivere verschiedenen durchaus sensiblen Bereichen (Inview I I)

In vielen Bereichen haben sich CSOs eine weitreichende Expertise für die Lösung gesellschaftlicher Probleme angeeignet, die auch in die Entwicklung von Dienstleistungen einfließt, wenn auch nicht immer in dem Maße, in dem es seiten der CSOs notwendig erscheint. Im Rahmen der Interviews wurde beispielweise erwähnt, dass Innovationen im sozialen Dienstleistungsbereich von de offentlichen Hand in Österreich nicht sehr gechâtzt werden, weil sie vorwiegend als Kostenfakentsprechende Advocacy-Arbeit leisten. Es besteh hohe Kompetenz, weil sie die Probleme und Anlietives Beispiel wurde der H. Als gegenteiliges posi-
Hier bestand hoher Handlungsbedarf, der zunächst on CSOs unter Einsatz von Spenden abgedeckt wurde. In weiterer Folge hat die öffentliche Hand jedoch die Finanzierung übernommen (Interview I I). Bei Ausschreibungen ist die Beteiligung im Vorfeld nur begrenzt möglich, weil diese ein Ausschlieren ist. Es gibt hier nur die Möglichkeit Berichtigungsfragen zur Leistungsbeschreibung zu stellen. Bei Dienstleistungen, die vom Auftraggeber nicht a priori beschrieben werden konnen, gibt es das Instrumentarium des Verhandlungsverfahrens, das den Input der Bieter berücksichtigt (Interview 9).

\section{VERBESSERUNGSPOTENZIALE}

Im Rahmen der Interviews wurden folgende Verbesiale genannt:

\section{An die Adresse der öffentlichen Han}

\section{r. Rahmenverträge}

wichtiges Thema ist der Wunsch nach größere Planungssicherheit durch mehrjährige RahmenHörderger Bïn mit CSOs sich da uf einstellen könnw. Es soltte keine Verträge ohne Indexklausel für personalintensive Dienstleistungsunternehmen geben, denn Personal in der Betreuung von anderen Menschen braucht Kompetenzen und muss motiviert seinc (Interview 3,8, II). Overheadkosten sollten bei Fördeingen ebenfalls ausreichend berücksichtigt werden.

\section{Definition von Qualitätsstandards}

Häufig genannt wurde auch der Wunsch nach ofensiverer Definition von Qualitätsstandards mit enhergehender Reduzierung der Regulierungen auf wesentliche Punkte zur Erfüllung von Qualität (Interview I I)

Âhnlich, wie im Gesundheitsbereich Zielsteuerungsvereinbarungen getroffen werden, sollte es auch bei anderen Dienstleistungen eine Definition von Qualität und eine klare Vereinbarung zwischen den unterschiedichen Geldgebern über die Auftei lung der Finanzierung geben (Interview 2). Es sollte . bei der Ubertragung von sozialen Dienstleistungen an private Dienstleister sicherstelt (Interview 8).
Weiters angeregt wurde der Einsatz eines Exertengremiums bei der Beurteilung der Qualität und des Preis-Leistungsverhälttnisses von Projekten (, nicht nur von Ministerialbürokratie) (Interview 5).

\section{Stärkung der Gemeinnützigke}

organisationen sollte bei der Verger ist, dass CSOs keine Überschüsse an Eigentümer ausschütten, sondern diese re-investieren. Weiters erzeugen sie einen gesellschaftlichen Mehrwert also Wirkungen, die über die Erbringung der Dienstleistung weit hinausgehen. Dies liegt im öffentlichen Interesse und sollte entsprechend gefördert und unterstützt werden. (Interview 2, 4, 8
Mögliche Gestaltung des Wettbewerbs/der Vergabekriterien:

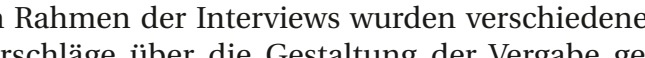
nannt:

Andere Verfahrensgestaltung: I. Feststellungserfahren, ob Organisation die Voraussetzung erfüllt 2. Vereinbarung über Leistungsaustauch 3. Kontrolle und Überwachung (Interview 8). Beauftragung von geeigneten Einrichtungen, die vorher ein Gütesiegel bekommen haben. Zulassungsverfahren - wer bestimmte Kriterie erfüllt, kann zu bestimmten Bedingunge (innerhalb einer gewissen Preisspanne) anbieten, Qualitätskriterien werden vorab definiert.

Wettbewerb im Markt, bei dem es ein Zulassungsverfahren gibt. Wer die Rahmenbedingungen erfüllt, kann in einem bestimmten Preisband anbieten. D.h. es gibt eine Konzentration auf die Regelung der Leistungen und Konditionen fur bestimmte Zielgruppen un pirive sollte möglich sein. Im Gegensatz din dect beim Wettbewerb um den Markt in Anbieter den gesamten Markt ab. Dadurch kommt Monopolstellung die Konkurrenz wird ausehöhlt (Interview I I).

\section{sichtigen}

Angeregt wurde auch die Schaffung von Auffangetzen für kleine Organisationen, beispielsweise ber die Finanzierung eines Sockelbetrags in Kombination mit leistungsbezogener Finanzierung (z. B. 列 政

Ergänzen) (Interview5). nterview 5)

\section{Klärung der Zuständigkei}

vielen Bereichen (z. B. Wohnen und Arbeit in de ehindertenhilfe) ist eine eindeutige Klärung de Zustândigkeiten (Bund/Land/Gemeinden) wünung beteiligt sind, sollte es eine bessere Abstimmung unter ihnen geben (Interview 2, 8).

\section{An die Adresse der CSOs:}

\section{- Interessenvertretung}

sen stärker bündeln un außen tragen, um die gemeinsamen Anliegen in der Öffentlichkeit besser zu vertreten (Interview

-


68 ANALYSE EER RAMMENEDINGWNGEN

8. Transparenz bei der Vergabe von Leistungsverträgen/Förderungen

ie Transparenz bei der Vergabe von LeistungsJahren ein wichtiges Thema geworden. Im Folge den werden Rahmenbedingungen und Themenfeder bei der Umsetzung analysier

\section{RECHTLICHE REGELUNGEN}

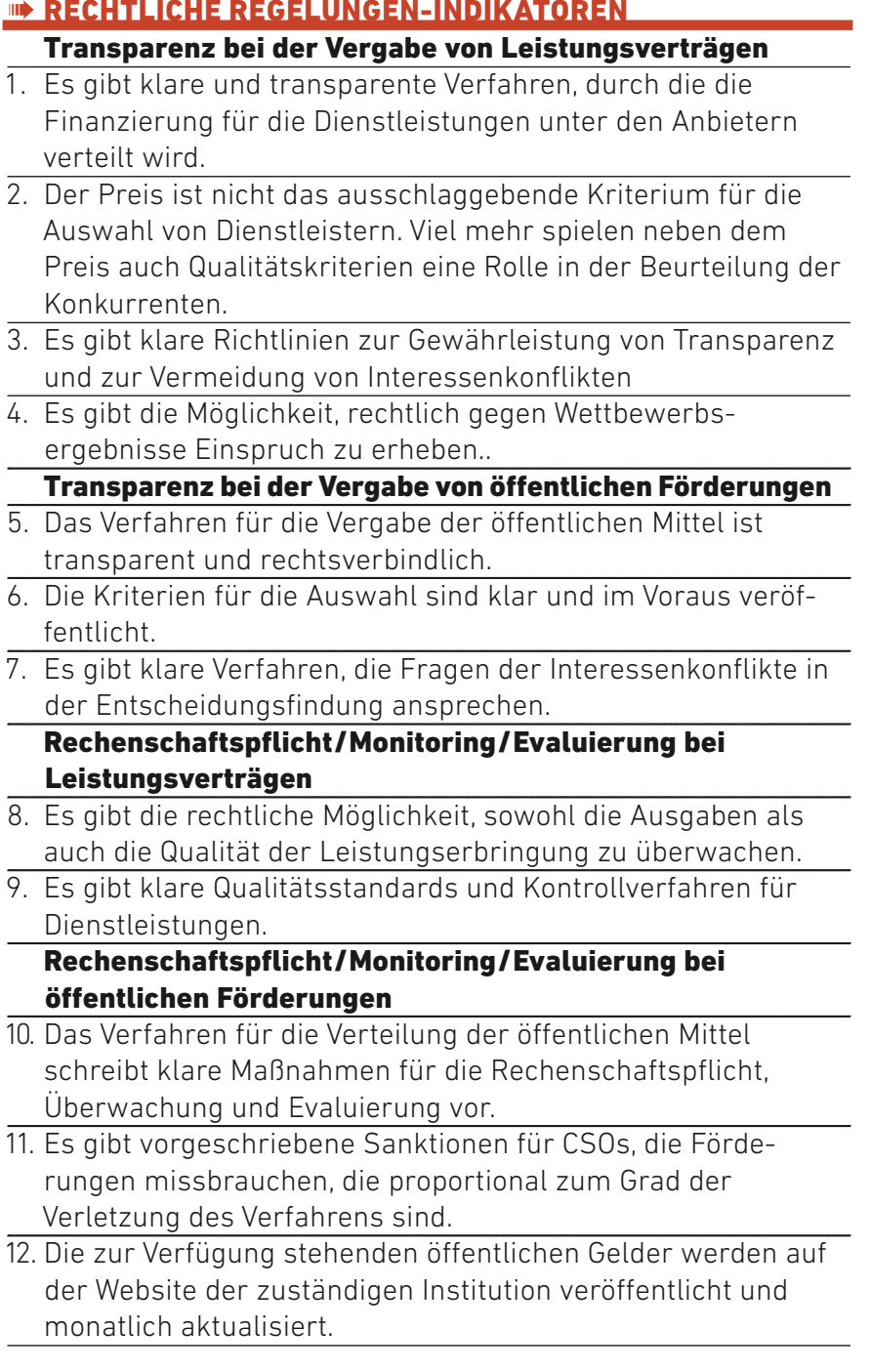

Das Vergaberecht unterscheidet nicht, ob entgeltliche Leistungen von Trägern der öffentlichen Hand, gewinnorientierten Unternehmen oder CSOs angerechtliche (Gewerberecht), technische (Ausstattung, MitarbeiterInnen, Referenzen) und die wirtschaftliche Leistungsfähigkeit (Auftragswert im Verhältnis zum Gesamtumsatz) (Interview 9). Ausschreibungen sind so zu gestalten, dass Interessenlagen weder benachteiligt noch bevorzugt werden, (\$ I9(I) Bundesvergabegesetz - Grundsatz der Gleichbehandlung aller Bieter in einem Vergabeverfahren), d. $h$. laut Gesetz geht es dem öffentlichen Auftraggebe um die bestmögliche Versorgung (Interview 9).

In Österreich gibt es eine Vielzahl an Förderungen von unterschiedlichen Fördergebern. Die Homepage des Bundesministeriums für Finanzen (BMF) ${ }^{\text {102 }}$ beziffert rund 2.600 Förderprogramme au Bundes- und 3.roo Förderprogramme auf Landes-
69

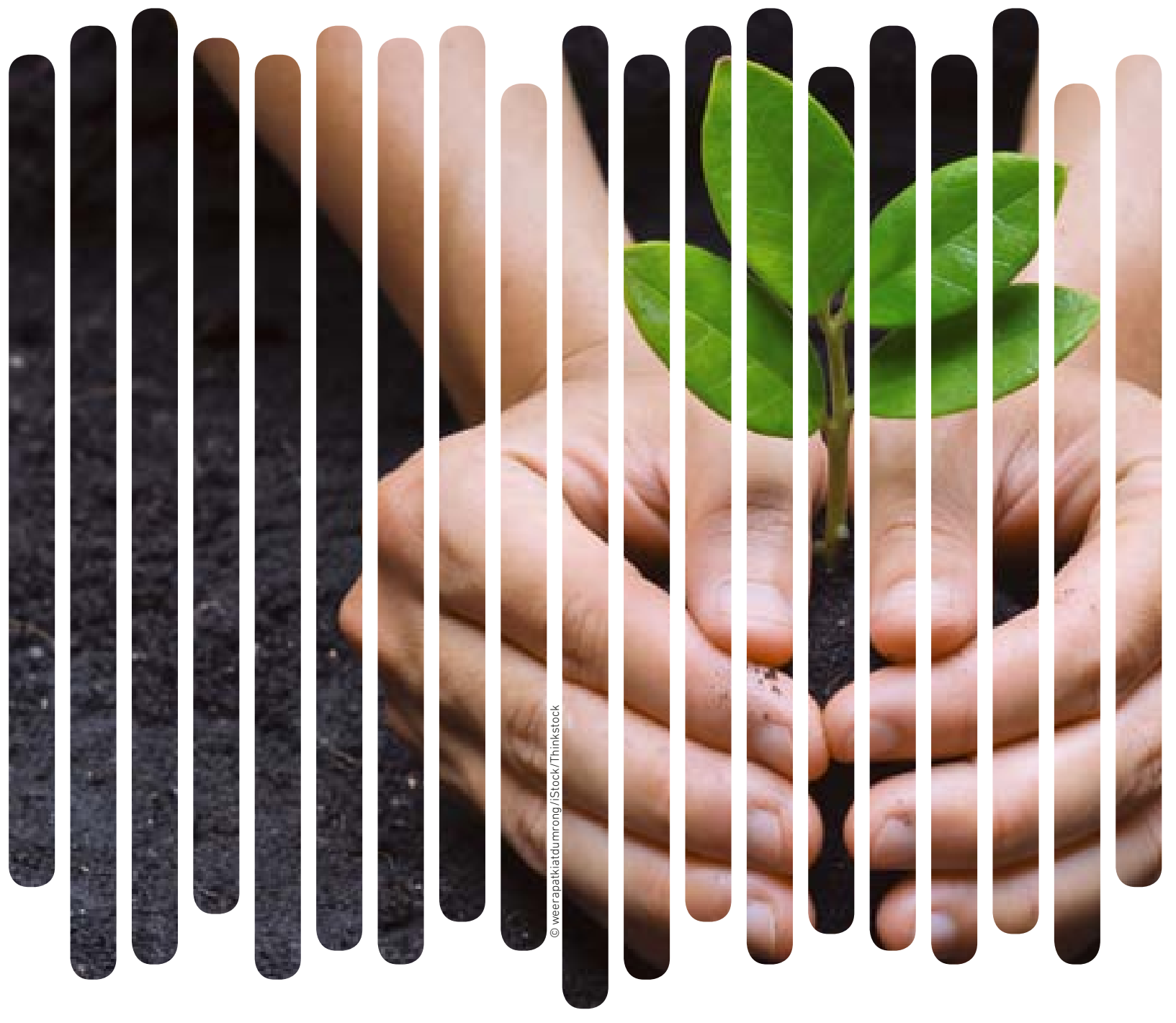

ebene. Die Förderrichtlinien und die Vergabeprozedere (Ausschreibung, Einreichung, Evaluierung) sind - je nach Fordergeber - sehr unterschiedlich gestaltet. Tendenziell erfüllen Förderrichtlinien die im Rahmen der Indikatoren genannten Kriterien (Klarheit der Auswahlkriterien, Nennung von Inte essenskonflikten, Vorgabe zu Maßnahmen der Re chenschaftspflicht, Uberwachung und Evaluation vorgeschrieben Sanktionen), dies ist jedoch nich er gleichermaßen der Fal.

Das Vergaberecht orientiert sich eng an den Richtlinien von Inter und Transpar unz be der verneidung nicht zur Diskriminierung komment, wenneske Nieder Der Tran ten im Vergaberecht - - sas Vergaberecht un tersche det zwischen Billigstbieter- und Bestbieterprinzip. Für die Auswabl von Dienstleistern kommen Preisund Qualitätskriterien zum Einsatz z . B. Erfahrung von erprobten Dienstleistern. Ein Einspruch beim Bundesvergabeamt ist möglich, jedoch sehr zeitintensiv.

Es bestehen klare Systeme der Rechenschaftspflicht, Öberwachung und Bewertung de Leistungserbringung mit Qualitätsstandards, die jedoch nicht unabhängig (von externer Stelle) überprüft werden (Interview 5, 8).
In Bezug auf Förderungen, gibt es in den letzten laut BMF) transparenter zu machen. Dafür wurde im Jahr 2013 ein Transparenzportal ${ }^{103}$ eingerichtet, das künftig in mehreren Stufen ausgebaut werden soll. Ziel ist es, eine einheitliche Darstellung aller bundesweit angebotenen Förderleistungen zu schaffen, Informationen über infrage kommende zukünftige Förderungen zu geben und den Einreichprozess zu unterstützen. Das Portal unterscheidet zwischen Leistungen/Förderungen fur Privatpersonen, Unter nehmen, NPOsund offentliche Einrichtungen. Nicht derungen Da das Transparenzportal inenoch relaivjung Maßnatme dist, sich nochim Aufbau befindet, gilt es abzuwarten inwieweit dis Truberenzhierkünftig bescer gewährleistet sein wird.

Zusätzlich gibt es auch verschiedene ichsweise Bestrebungen, die Transparenz und Klarheit bei Förderungen zu erhöhen. Beispielsweise ist es Ziel des neuen Bundes-Sportförderungsgesetzes, Förderungen klar zu definieren, unter anderem um Mehrfachförderungen zu vermeiden (Interview I).

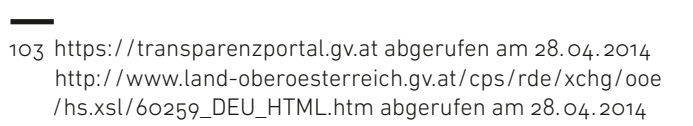

BESONDERHEITEN IN DER PRAXIS

(II) PRAXIS-INDIKATOREN

Transparenz bei der Vergabe von Leistungsverträgen $\frac{1 . \text { Für viele Dienstleistungen werden CSOs beauftragt. }}{2 \text {. Die Wettbewerbe werden als fair betrachtet und Interessen- }}$ konflikte werden vermieden.

Transparenz bei der Vergabe von öffentlichen Mitteln 3. Informationen zu den Verfahren für die Förderungen und Informationen über die geförderten Projekte sind öffentlich verfügbar.

4. Entscheidungen über die Vergabe von Fördermitteln (pro, contra oder nur teilweise) müssen entsprechend den Ausschreibungskriterien transparent und nachvollziehbar

begrundet werden.

作 den verschiedenen Fordergebern in abgestimmter Weise an. 7. Entscheidungen für Angebote werden als fair betrachtet und

Förderungen vorgetegt, der die Summe die ber erfolgten Förderungen vorgelegt, der die Summe, die begünstigte 8. Es gibt die rechtliche Möglichkeit, sowohl die Ausgaben als auch die Qualität der Leistungserbringung zu überwachen. Rechenschaftspflicht/Monitoring/Evaluierung bei Leistungsverträgen 10. CSOs unterliegen nicht der übermäßigen Kontrolle. bekannt gegebenen Verfahren und Kriterien durchgeführt.

Effekte/Wirkungen der Leistungen, die offentlich zugänglich

Rechenschaftspflicht/Monitoring/Evaluierung bei öffentlichen Förderungen

12. Die Überwachung erfolgt kontinuierlich und in Ubereinstim$\frac{\text { mung mit vorher definierten und objektiven Indikatoren. }}{13 . \text { Es wird von staatlichen Stellen eine regelmäßige Bewertung }}$ von Verwendung/Wirkungen der öffentlichen Mittel durchgeführt und diese ist öffentlich zugänglich.

CSOs werden mit einer Vielzahl an Dienstleistun gen beauftragt, einerseits in Form von Leistungsverträgen, andererseits in Form von Förderungen Im Rahmen der Interviews wurde öfters erwähnt, dass die Vergaben nicht immer als fair erachtet wurden, sondern, dass es Hnweise auf die politische Bevorzugung bestimmter Auftraggeber gibt, rels geistun bungskriterien abgewichen wurde (Interview 5 ). Oftmals entstand auch der Eindruck Ausschreibung auf das Prefil ter beck, dass die bieter zugeschnitten war, so dass Mitbieter keine realistische Chance hatten, den Zuschlag zu erhalten, andererseits haben CSOs selbst schon manchmal von einer solchen Vorgehensweise profitiert (Interview 4). Angemerkt wurde weiters, dass es auf nationaler Ebene eindeutige Mechanismen für die Verteilung öffentlicher Mittel an CSOs nach Par- 
teieninteressen (SPÖ und ÖVP) gibt (Interview 5, VERBESSERUNGSPOTENZIALE

8). Es gibt zunehmende Transparenz über die Vergabe von Förderungen und deren Höhe bei den einzelnen Förderprogrammen. Wenig bis kein Transparenz gibt es über die Entscheidungen, zu welchen Konditionen Aufträge letztlich vergeben wurden und warum ein Auftragnehmer den Auftrag erhalten hat (Interview 5). Defizite werden auch bei Absagen geortet. Es wird oft nicht anhand der Ausschreibungskriterien schlüssig begrün

Bei der Finanzierung von Dienstleistunge durch verschiedene Zahlungsgeber (insbesondere Gemeinden/Länder/Gemeinden/Sozialversicherungsträger) treten häufig Abstimmungsprobleme auf. Jeder Zahlungsgeber versucht sein eigenes Subsystem zu optimieren und die CSOs werden bei Ver-
handlungen jeweils auf die anderen verwiesen (Interview 2). Aufwändig ist bei Mehrfachförderunge auch die Abrechnung, da es häufig vorkommt, dass die Fördergeber unterschiedliche Abrechnungssyseme und -logiken haben (Interview 5, 8)

Der Bund veröffentlicht jährlich einen Fö derungsbericht, der dem Nationalrat vorgele wir und in dent alle subventienter Verfügung Das Land Oberösterreich veröffentlich beispielsweise seine ausbezahlten Förderungen in Internet wo - strukturiert nach verschiedenen Tatigkeitsbereichen - der Förderzweck die Höhe der Förderungen (ab 4.000 Euro) sowie die Institutionen, die diese erhalten haben, ausgewiesen sind. In Bezug auf die Rechenschafts- und Evaluierungspflichten wurde vielfach angemerkt, dass diese überzogen sind. Die Rechnungslegung wird als sehr zeitintensiv und mühsam beschrieben. Es besteht zwar Verstandnis dafür, dass kontrollie werden muss, allerdings stehe der Aufwand dafür nicht mehr im Verhâltnis zur Leistung, die erbrach wird: : Der Aufwand fur die Nachweise ist fast intensiver und großser als die Erbringung der Dienstleistung und das kann es nicht sein.، (Interview 8). Es gebe zu viele Kontrollen, auch von extornen Prüfern, die Abrechnungssysteme seien kompliziert und aufwändig (Interview I , 8, 5).

Generell besteht ein starker Legitimationsdruck für CSOs. Lange Zeit lag der Fokus darauf, wie CSOs die ihnen zu Verfügung gestellten finanziellen Mittel verwenden. In jüngerer Zeit finden erzielte Wintzlich privis vist, weil nicht nur due, was grid, wie viele Kosten CSOs vicht narest auch, welche Wirkungen durch sie erzielt werden. Allerdings sind der Wirkungsmessung und dem Vergleich von Wirkungen - abhängig vom Aufgbenfeld - auch Grenzen gesetzt. So lässt sich beispielsweise die Nachhaltigkeit der Schulungsmaßnahmen bei Menschen mit Behinderung nur schwer messen und nicht mit jener von Arbeitslosen vergleichen (Interview 8).

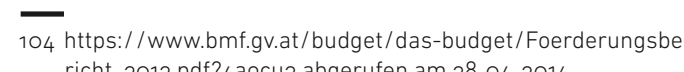

m Rahmen der Interviews wurden folgende Verbesungspotenziale genannt:

Vielfaltige Vorschläge gab es hinsichtlich der Gestaltung der Qualitätskontrolle. Der Grundtenor der Interviews ging dahin, den CSOs wieder meh zu vertrauen, die Rechenschaftspflicht zu vereinfachen und strenge Kontrollen auf Stichproben zu reduzieren, >ohne dass sie an Substanz verliert (Interview 8, II). Durch eine Vereinheitlichung sollte es zu einer Vereinfachung in der Bürokratie kommen daje nach Forderstelle unterschiedliche Vorschriften, Bearbeitungsweisen und Rechnungslegungvorschiften zu berücksichtigen sind, was unnötiAufwand verursacht.

Für die Kontrolle der Leistungserbringungen sollten verstärkt auch unabhängige Stellen eingesetzt werden, die eine entsprechende Expertise aufise unter Einbindung von NPO nterview 5, 8).

Die Transparenz sollte grundsätzlich ausgebaut werden durch

aktuelle Information über die zur Verfügung den Förderberei-

Transparenz bei Entscheidungen und bei den

Entscheidungskriterien

Q

Veröffentlichung von Abschlussberichten

(zumindest in Kurzfassung) um Lerneffekte für

weitere Projekte zu ermöglichen.

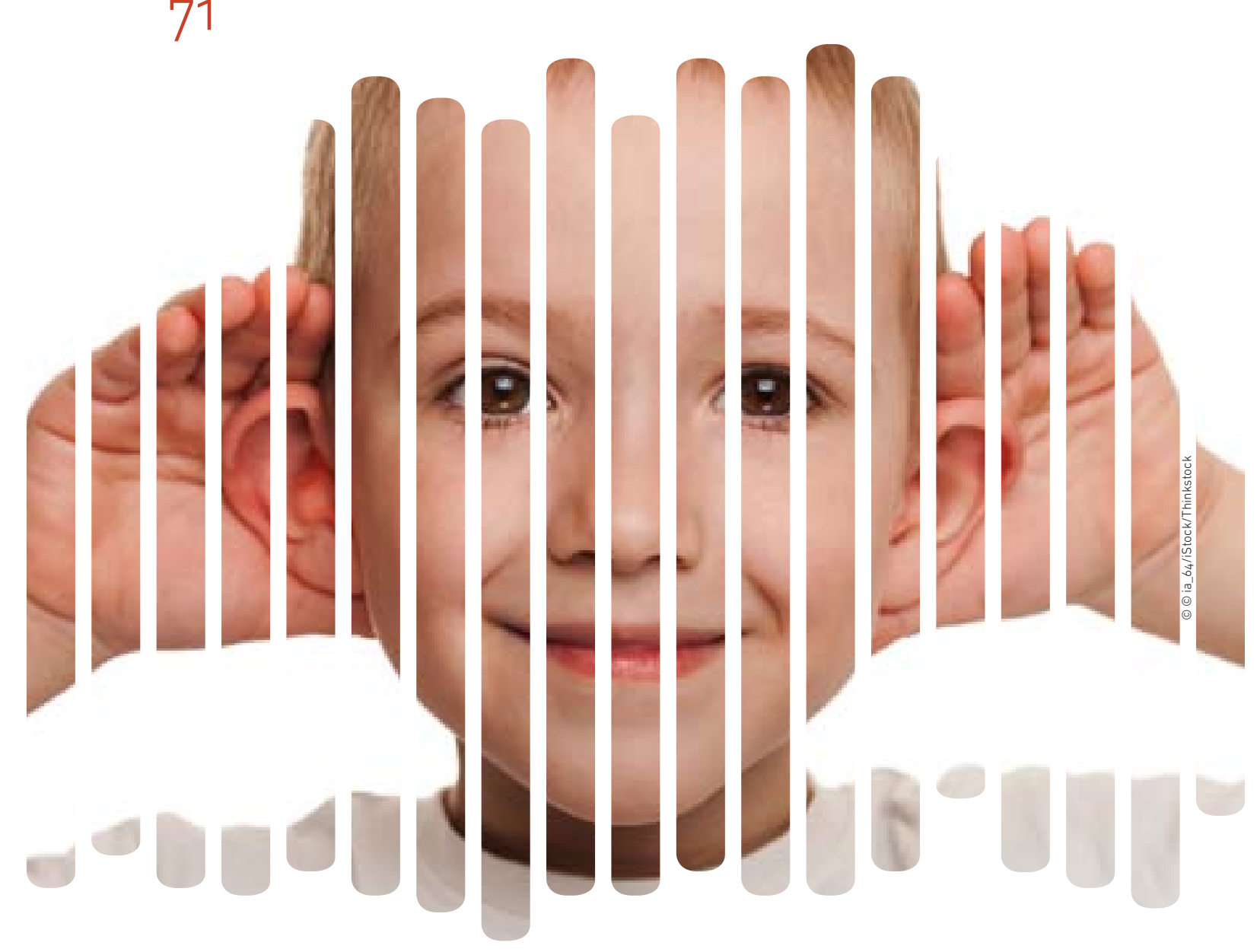

9. Wahrnehmbarkeit zivilgesellschaftlicher Wirkungen

er Wissensstand und die Einstellung der österreichischen Bevölkerung zu NPOs bzw. gemeinnützigen Organisationen stellt eine wichtige Bedingung für die Arbeit dieser Organisationen dar. Sowohl ehrenamtliches Engagement, Spendenbereitschaft als auch sâmtliche andere Formen der Unterstützung werden davon beeinflusst.

Bisher ist jedoch noch sehr wenig über die öfentliche Wahrnehmung des gesamten Sektors in Österreich bekannt. Daher wurde entschieden, im Rahmen des Civil Society Index - Rapid Assessmen dieses Thema genauer zu beleuchten.

In der Erhebung geht es um

die Wahrnehmung von gemeinnützigen bzw. Non-Profit-Organisationen in der österreichischen Bevölkerung

eine Erfassung des Wissensstands über NPO somantionskanäle

,ie NPOs in Österreich entgegengebăzung eine Finschätzung der zukïnftigen Entwik-

lungen für NPOs in Österreich

Potenziell genutzte Informationsquellen über gemeinnützige

\section{WAHRNEHMBARKEIT - INDIKATOREN}

Persönlicher Wissensstand über gemeinnützige bzw.

Zugemessener Stellenwert von gemeinnützigen bzw.

Als wichtig eingeschatzte Wirkungsbereiche von gemein-

bzw. Non-Profit-Organisatios

7. Persönlicher Kontakt mit gemeinnützigen bzw.

Non-Profit-Organisationen und Art dieses Kontakts

8. Entwicklung der Bedeulung von gen

Wahrnehmung möglicher zukünftiger Unterstützungen

für NPOs

Zur Beantwortung der Fragestellungen wurde beim Marktforschungsinstitut market eine repräsentative Telefonumfrage in Auftrag gegeben. Die in der Folge dargestellten Informationen und Ergebnisse wurden
entnommen

Methode: Zielgruppe der Erhebung war die gesamtösterreichische Bevölkerung ab I8 Jahren. Es wurde im Sinne der Repräsentativität der Studie eine Kombination aus Random- und Quotaauswahl angewendet.

Telefonische Interviews mittels Fragebogen wurden als amzielführendsten eingestuft.Zum Ersten, 
weil damit genügend Zeitfür eine Vertiefung des The- I8 - 29 Jahre) genannt. Diese Assoziation ist dort mit mas vorhanden ist. Zum Zweiten, weil wir damit sichergehen konnten, Menschen, die über OnlineUmfragen nicht erreichbar wären (meist die ältere Bevölkerungsgruppe), nicht auszuschließen. DieUmfrage wurde von I6. bis 24. April 2014 unter 40I TeilnehmerInnen durchgeführt und hat eine maximale statistischeSchwankungsbreitevon $+/-4,99$ Prozent.

\section{ZUSAMMENFASSUNG DER ERGEBNISS}

Jensstand úber gemeinnútzige bzw. Non-Profit-Organisationen kann als gut bezeichnet werden. 70 Prozent der Befragten meinen über die Ziele, Aufgaben und Tätigkeiten von gemeinnützigen bzw. Non-Profit-Organisationen 'gut

oder sogar `sehr gut ‘ Bescheid zu wissen.

Genauer betrachtet sind es jedoch nur 29 Prozent, die sich als sehr gut informiert einstufen un 40 Prozent, die meinen 'gut informiert zu sein. Die-
ses Ergebnis lässt weiteren Informationsbedarf erkes Ergebnis lässt weiteren Informationsbedarf erBefragten, das von einem eher geringen Wissen
stand über NPOs spricht. Im urbanen Bereich wird derWissensstand etwas schlechter als in ländlichen Gebieten wahrgenommen. Gleichzeitig lässt sich wenig überraschend - eine positive Korrelation mit dem Bildungsniveau erkennen.

\section{Abbildung}

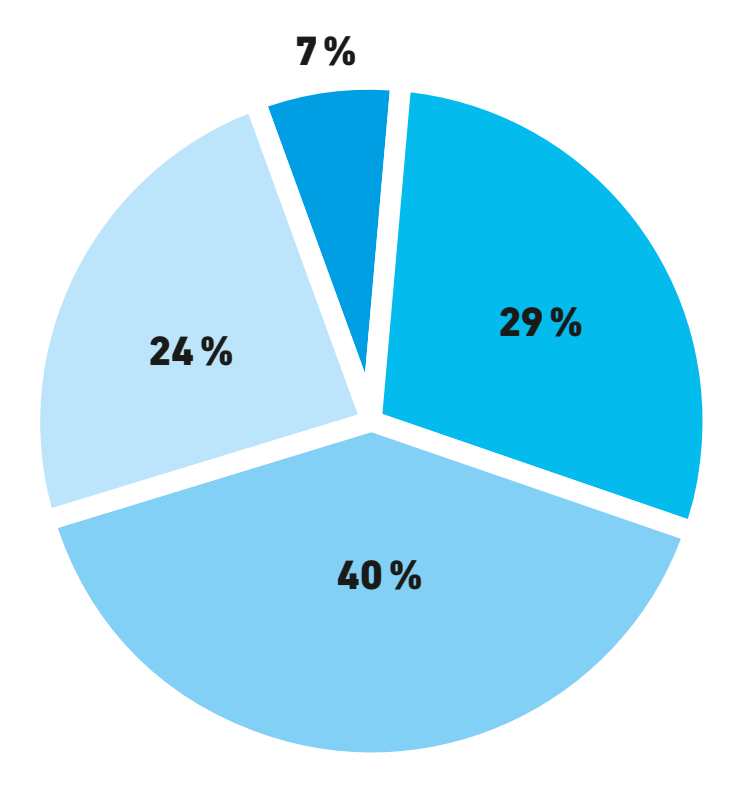

Quelle: market-Umfrage 2014

Spontane Assoziationen zu gemeinnützigen bzw. Non-Profit-Organisationen gibt es vor allem mit unmittelbarer Hilfe. Jeder Dritte verbindet mit gemeinnǘtzigen Organisationen Begriffe wie
,Menschen helfen, , Hilfe für Mitmenschen $`$ oder ,Hilfe für Arme`. Weitere 30 Prozent denken dabei an konkrete Organisationen wie z. B. die Caritas, das Rote Kreuz oder die Freiwillige Feuerwehr. Das Thema /Freiwilligkeit und ehrenamtliches Engagement wird vor allem von jüngeren Menschen (von zent fast doppelt so hoch wie beim Rest de

Grundlegende Informationen über gemein meisten Befrag-Profit-Organisationen holen die mit FreundInnen und Bekannten ein. Dieses Inforternetrecherche (google o. ä.). Fernsehen und Radio kommen als potenzielle Informationskanäle an Aktivitäten auf. Auffällig ist dabei eine größere Neigung zu Zeitungsberichten oder postalischen $\mathrm{Zu}$ sendungen im Osten Osterreichs (Wien nisationen noch nicht durchgesetzt, nur I2 Prozen Dich daruber zu informieren.

Die Bedeutung von gemeinnützigen bzw wird in ÖstOrganis wire (a) und kaum Menschen die die Rolle von NPOs gering schätzen. Die zehn Prozent der Befragten, die den schätzen. Die zehn Prozent der Befragten, die den man unter gekehrt lässt dies den Schluss zu, dass jene, die über mit ihnen vertraut sind, die Wichtigkeit von NPOs als hoch einstufen.

sehr gut

gut

eher weniger mationsverhalten wird gefolgt von allgemeiner Indritter Stelle: 28 Prozent geben an sich darüber zu

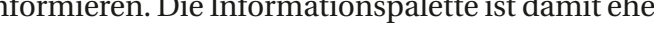
Burgenland, Niederosterreich). Social Media hat sich beim Thema NPOs bzw. gemeinnützige Orgagemeinnüziger Einrichtungen. Man fndetnahezu gemeinnützige Einrichtungen Bescheid wissen und

so gut wie gar nicht
Abbildung 5:

Gesellschaftlicher Stellenwert von NPOs

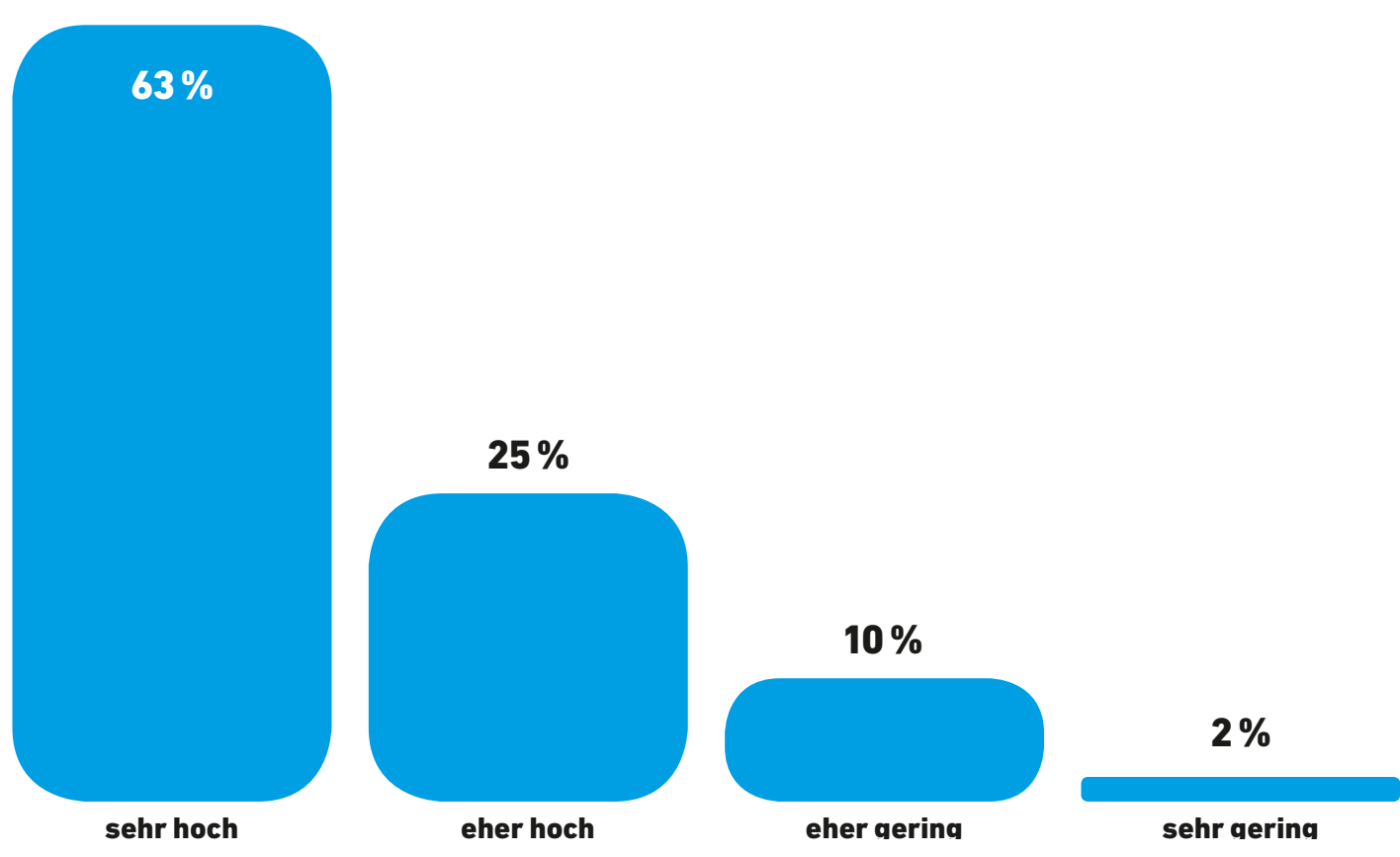

Quelle: market-Umfrage 2014

Außerdem wurde in der Befragung die in der österreichischen Bevölkerung subje dene Bedeutung der einzelnen Wirkungsbereiche von NPOs untersucht. Hier konnten ,Gesundheit und soziale Dienster mit 80 Prozent als führend identifiziert werden. Gefolgt vom Thema ,Familie, Kinder und Jugend $: 73$ Prozent sehen hier eine besonders wichtige Rolle von NPOs. Von jüngeren Menschen (bis 29 Jahre) werden NPOs bei den Themen Menschenrechte (64 Prozent) und Bildung (57 Prozent) als wichtig eingestuft. Vor allem Jüngere bis 50 Jahre messen Einrichtungen im familiären Bereich hohe Relevanz zu.

Beim Thema Menschenrechte und Entwicklungszusammenarbeit lässt sich insofern ein bildungsbedingter Zusammenhang erkennen, als Personen mit höherem Bildungsgrad den Wert von NPOs in diesen Bereichen hơher einschätzen.

Die Wahrnehmung des Nutzens von gemeinnützigen bzw. 64 Prozent der befragten stimmen svoll und ganz die Geslo NPOs ( Enthesungsuntion für den Staat durch NPOs wahr. Weniger präsent ist die Sontellfultion fü Wirtschaft und Stats. Wahrscheinlich weil generell und vor allem bei den Jüngeren an der politischen Unabhängigkeit v vieler Organisationen gezweifelt wird. Einmal mehr bestätigt sich auch, dass besser Informierte den Nutzen von gemeinnützigen Organisationen stärker erkennen als weniger gut Informierte.
85 Prozent der ÖsterreicherInnen hatten in der Vergangenheit in irgendeiner Form persönlichen Kontakt mit gemeinnützigen Organisationen. Organisationen mit einem lokal weit verzweigten Freiwillige Feuerwehr oder auch die Caritas werden hier am häufigsten angeführt. Bei der Altersgruppe zwischen zo und 49 Jahren hatten sogar 92 Prozent bereits direkten Kontakt mit NPOs. Bei durchschnittlich 70-80 Prozent der Kontakte geht es um fenzentersonen, die angeben mit dem Roten Kreuz, der Caritas oder der freiwillgen Feuerwehr Kontakt gehabt zu haen, arbeiteten ca. 30 Prozent bereits aktiv mit. 


\section{ZUKUNFTSENTWICKLUNG:}

Mehr als jeder Zweite erwartet, dass der Stellenwert von NPOs zunehmen wird. Interessanterweise zeig sich das insbesondere im urbanen Bereich, der sich zwar generell über NPOs etwas schwächer informiert zeigt, aber steigenden Bedarf nach deren Leistungen in den Städten erwartet.

Wenn es um mögliche zukünftige Unterstützung für NPOs geht, sprechen sich 67 Prozent voll und ganz für ' steuerliche Begünstigung a aus und 23 Prozent vergeben diesbezüglich die Note 2. Insgesamt stehen damit 9o Prozent dieser Form der Unterstützung positiv gegenüber.

Etwas weniger, nämlich 6I Prozent befürworten , direkte Förderungen`durch den Staat voll und ganz. Die Note 2 wird hier von 27 Prozent vergeben. Insgesant stehen danit auch der Unterstutzung durch direkte Förderungen fast 90 Prozent posit gegenüber.

Man kann hier auf einzelne Alterspräferenzen herunter brechen: Jüngere tendieren ein wenig stärrärker für Ste stärker für

Bezüglich eines stärkeren Mitspracherechts bei politischen Entscheidungen sind die Befragten zurückhaltender. Trotzdem würden immerhin fast

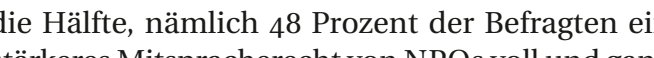
befürworten. Weitere 23 Prozent vergeben die Note 2. Damit signalisieren 7I Prozent auch bei diesem

abbildung 6 :

Wahrnehmung möglicher zukünftiger Unterstützung für NPOs

\section{$23 \%$}

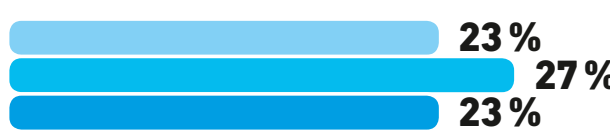$$
17 \%
$$
$4 \%$

überhaup mehr direkte Förderungen

mehr Mitspracherecht im politischen Entscheidungsprozess
IV.

RAHMENBEDINGUNGEN FÜR DIE ZIVILGESELLSCHAFT IN ÖSTERREICH STARKEN UND SCHWÄCHEN

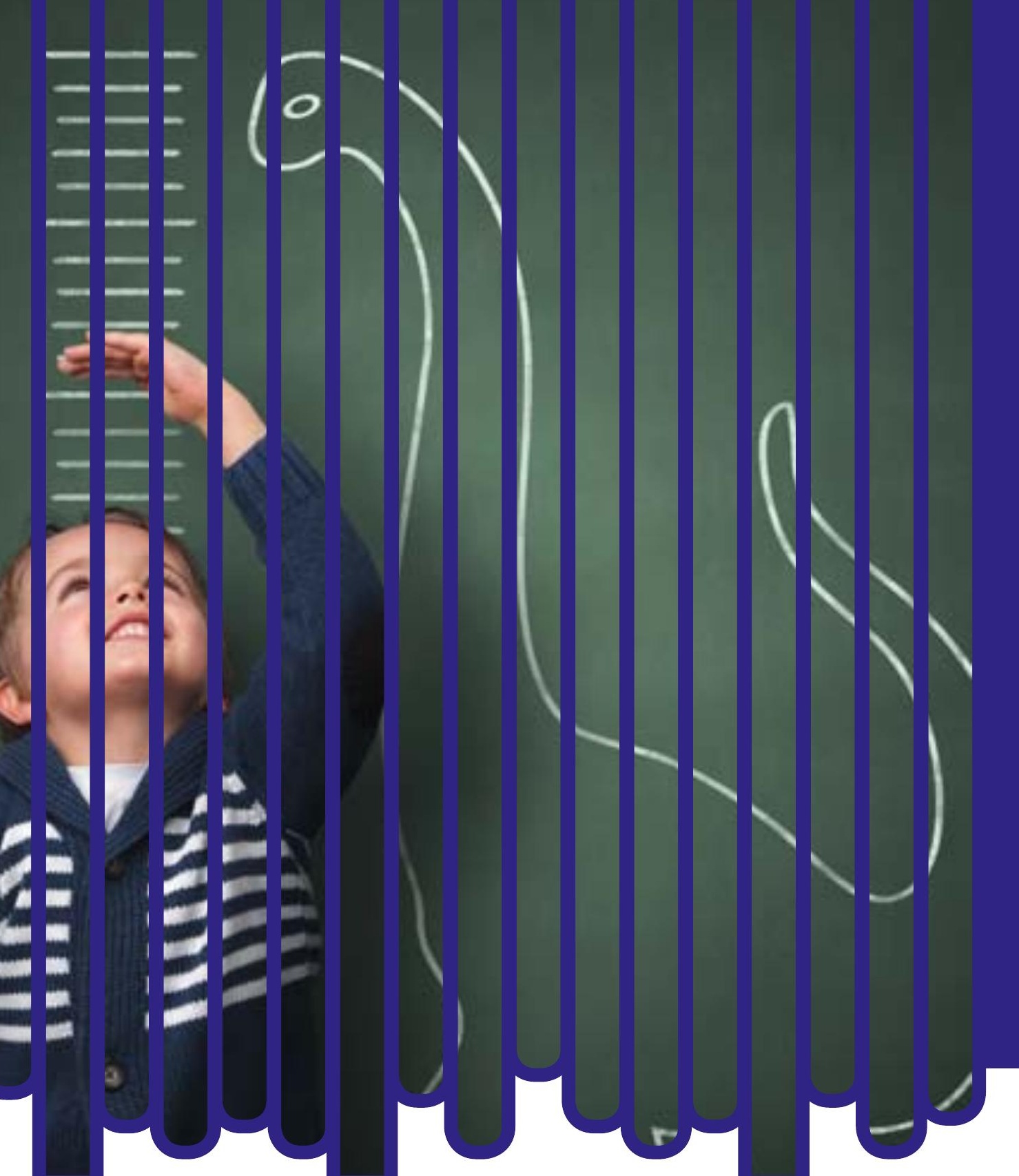




\section{RAHMENBEDINGUNGEN FÜR DIE ZIVILGESELLSCHAFT IN ÖSTERREICH - STÄRKEN UND SCHWÄCHEN}

\begin{tabular}{|c|c|c|}
\hline & STARKEN & SCHWÄCHEN \\
\hline Demokratie & $\begin{array}{l}\text { + Garantie auf Auslebung der } \\
\text { individuellen Freiheiten } \\
\text { + Rechtsstaatlichkeit: effektives } \\
\text { Rechtssystem, Vertrauen in das } \\
\text { Justizsystem, Gleichheit vor dem } \\
\text { Gesetz } \\
\text { + freier Wettbewerb um } \\
\text { Wählerlnnenstimmen } \\
\text { + Kontrolle: gutes System von } \\
\text { checks and balances }\end{array}$ & $\begin{array}{l}\text { - hohe Medienkonzentration } \\
\text { - Exklusivität des Wahlrechts bezogen } \\
\text { auf Staatsbürgerschaft } \\
\text { - geringes Vertrauen in Regierung, } \\
\text { Parteien und Parlament (wenn auch } \\
\text { im internationalen Vergleich über- } \\
\text { durchschnittlich hoch) } \\
\text { - schwache Einbindung von CSOs in } \\
\text { Politikprozesse }\end{array}$ \\
\hline$\overline{\text { Vereinigungsfreiheit }}$ & $\begin{array}{l}\text { + Etablierter Rechtsrahmen zur } \\
\text { Ausübung der Vereinigungsfreiheit } \\
\text { + Vorteilhafte Regelungen für } \\
\text { bestimmte registrierte Vereini- } \\
\text { gungen in Bezug auf Haftung, Steu- } \\
\text { erbegünstigungen und Spendenab- } \\
\text { setzbarkeit }\end{array}$ & $\begin{array}{l}\text { - strafrechtliche Bestimmungen, v. a. } \\
\text { § } 278 \text { a StGB } \text { Kriminelle Vereinigunge } \\
\text { können zivilgesellschaftliches } \\
\text { Engagement einschränken } \\
\text { - wenig Anreize für die Gründung von } \\
\text { gemeinnützigen Privatstiftungen }\end{array}$ \\
\hline Versammlungsfreiheit & $\begin{array}{l}\text { + Das Grundrecht auf } \\
\text { Versammlungsfreiheit basiert auf } \\
\text { internationalen Standards gemäß } \\
\text { der Europäischen Menschenrechts- } \\
\text { konvention } \\
\text { + Das Versammlungsrecht wird durch } \\
\text { die Behörden meist nicht } \\
\text { beschränkt sondern geschützt } \\
+ \text { Versammmlungen müssen nicht } \\
\text { bewilligt werden, es bedarf lediglich } \\
\text { einer Nicht-Untersagung }\end{array}$ & $\begin{array}{l}\text { - Versammlungsgesetz, Staatsgrund- } \\
\text { gesetz und Art. } 11 \text { der EMRK } \\
\text { harmonieren nicht miteinander und } \\
\text { sind z. T. widersprüchlich } \\
\text { - Zeitgerechte Beschwerdemecha- } \\
\text { nismen bei Untersagungen fehlen } \\
\text { - Maßnahmenbeschwerden wegen } \\
\text { unverhältnismäßiger (Gewalt-)Hand- } \\
\text { lungen der Polizei sind mit relativ } \\
\text { hohen Kostenrisiken verbunden } \\
\text { - Strafrechtlicher Vorwurf des Land- } \\
\text { friedensbruchs (§274 StGB) und } \\
\text { Haftungsrisiken für Veranstalte- } \\
\text { rlnnen stellen eine Bedrohung dar }\end{array}$ \\
\hline $\begin{array}{l}\text { Zugang zu finanziellen } \\
\text { Ressourcen }\end{array}$ & $\begin{array}{l}\text { + grundsätzlich offener Zugang zu } \\
\text { vielfältigen Finanzierungsquellen } \\
\text { + Erhalt von Spenden aus In- und Aus- } \\
\text { land von Einzelpersonen und Unter- } \\
\text { nehmen möglich }\end{array}$ & $\begin{array}{l}\text { - Schwierigkeiten bei der Aufnahme } \\
\text { von Fremdkapital durch Vorschriften } \\
\text { zur Bewertung der Kreditwürdigkeit } \\
\text { Social Banking als Alternative noch } \\
\text { wenig etabliert } \\
\text { - teilweise Liquiditätsprobleme durch } \\
\text { späte Zahlungen durch öffentliche } \\
\text { Hand } \\
\text { - fehlender Nachweis der Gemein- } \\
\text { nützigkeit kann zu Problemen bei } \\
\text { Spenden aus dem Ausland führen } \\
\text { - nur wenige gemeinnützige Privat- } \\
\text { stiftungen im Vergleich zu anderen } \\
\text { Ländern }\end{array}$ \\
\hline $\begin{array}{l}\text { Steuerbegünstigung } \\
\text { für CSOs }\end{array}$ & $\begin{array}{l}\text { + Steuerliche Begünstigungen für } \\
\text { CSOs, sofern sie ausschließlich und } \\
\text { unmittelbar gemeinnützige, mild- } \\
\text { tätige oder kirchliche Zwecke } \\
\text { erfüllen } \\
\text { + CSOs dürfen - unter bestimmten } \\
\text { Voraussetzungen - wirtschaftliche } \\
\text { Aktivitäten entfalten } \\
\text { + Steuerliche Abzugsfähigkeit für } \\
\text { Privat- und Unternehmensspenden } \\
\text { an begünstigte CSOs }\end{array}$ & $\begin{array}{l}\text { - Keine behördliche (ex ante) Fest- } \\
\text { stellung der steuerlichen Begüns- } \\
\text { tigung von CSOs } \\
\text { - Mangelnde Rechtssicherheit bei der } \\
\text { (ex post) Beurteilung der steuer- } \\
\text { lichen Begünstigung durch die } \\
\text { Abgabenbehörden } \\
\text { - Steuerliche Abzugsfähigkeit ist auf } \\
\text { einige wenige begünstigte Zwecke } \\
\text { beschränkt } \\
\text { - Kaum steuerliche Anreize für die } \\
\text { Errichtung gemeinnütziger } \\
\text { Stiftungen } \\
\end{array}$ \\
\hline
\end{tabular}

\begin{tabular}{|c|c|c|}
\hline & & SCHWÄCHEN \\
\hline $\begin{array}{l}\text { Beschäftigung und } \\
\text { Freiwilligenarbeit }\end{array}$ & $\begin{array}{l}\text { + CSOs werden grundsätzlich wie } \\
\text { andere Arbeitgeber behandelt, } \\
\text { Steuererleichterungen (Kommunal- } \\
\text { steuer) für ausgewählte gemein- } \\
\text { nützige/mildtätige Vereine } \\
\text { + Kollektivverträge wie BAGS und } \\
\text { Rettungsdienste (aber nicht für alle } \\
\text { CSOs) } \\
\text { + Freiwilligengesetz bietet rechtliche } \\
\text { Grundlage für ausgewählte Formen } \\
\text { des freiwilligen Engagements }\end{array}$ & $\begin{array}{l}\text { - Gefahr der Prekarisierung der } \\
\text { Arbeitsverhältnisse durch unsichere } \\
\text { Finanzierungssituation bei CSOs } \\
\text { - Mangelnde Rechtssicherheit bei } \\
\text { diversen Engagementformen (z. B. } \\
\text { soziales Engagement im Ausland) } \\
\text { - Haftpflicht- und Unfallversicherung } \\
\text { für viele freiwillige Tätigkeiten nicht } \\
\text { gewährleistet }\end{array}$ \\
\hline $\begin{array}{l}\text { Bereitsteller von } \\
\text { Dienstleistungen }\end{array}$ & $\begin{array}{l}\text { + CSO sind grundsätzlich gleich- } \\
\text { berechtige Anbieter von Leistungen } \\
\text { in CSO-relevanten Bereichen, wie } \\
\text { Bildung, Gesundheit und Soziales } \\
\text { + Öffentliche Mittel werden für CSOs } \\
\text { durch Förderungen und } \\
\text { Leistungsverträge vergeben }\end{array}$ & $\begin{array}{l}\text { - Verhandlungen zwischen CSOs und } \\
\text { öffentlicher Hand oft nicht auf } \\
\text { gleicher Augenhöhe, starke Ein- } \\
\text { mischung in den Prozess der } \\
\text { Leistungserstellung } \\
\text { - teilweise hohe ökonomische Risiken } \\
\text { durch fehlende Indexanpassung } \\
\text { - Einschränkungen bei der Finanzie- } \\
\text { rung von Overheadkosten } \\
\text { - fehlende Planungssicherheit durch } \\
\text { einjährige Verträge, langsame } \\
\text { Entscheidungsprozesse, späte } \\
\text { Zahlungen }\end{array}$ \\
\hline $\begin{array}{l}\text { Transparenz bei der } \\
\text { Vergabe von } \\
\text { Leistungsverträgen und } \\
\text { Förderungen }\end{array}$ & $\begin{array}{l}\text { + Vergaberecht baut auf Gleich- } \\
\text { behandlung und Transparenz auf } \\
\text { + zunehmende Bemühungen um } \\
\text { Transparenz bei der Vergabe von } \\
\text { Förderungen }\end{array}$ & $\begin{array}{l}\text { - teilweise politisch bedingte Bevor- } \\
\text { zugung von BieterInnen/Förder- } \\
\text { nehmerlnnen } \\
\text { - nach wie vor wenig Transparenz bei } \\
\text { der Begründung von Absagen }\end{array}$ \\
\hline
\end{tabular}

\section{WAHRNEHMBARKEIT ZIVILGESELLSCHAFTLICHER WIRKUNGEN}

+ORKterreicherlnnen fühlen sich $\quad$ - Keine einheitlichen Statistiken über $\begin{array}{ll}+ \text { ÖsterreicherInnen fühlen sich } & - \text { Keine einheitlichen Statistiken } \\ \text { über CSOs gut informiert. } & \text { Leistungen des CSO Sektors }\end{array}$ + $88 \%$ der Bevölkerung erkennen den - Wenig gesichertes Wissen über die Nutzen von CSOs und schreiben Wirkungen der Zivilgesellschaft bzw. hohen Stellenwert zu. CSOS

+ $90 \%$ der OsterreicherInnen spre- - Primäre Assoziation zu NPOs lautet chelen" - wenig Bewusstsein über

Förderung von NPOs aus

$+70 \%$ der Österreicherlnnen wollen Zivilgesellschaft.

NPOs mehr Mitsprache bei

politischen Entscheidungs-

prozessen einräumen 
V.

HANDLUNGS-

EMPFEHLUNGEN

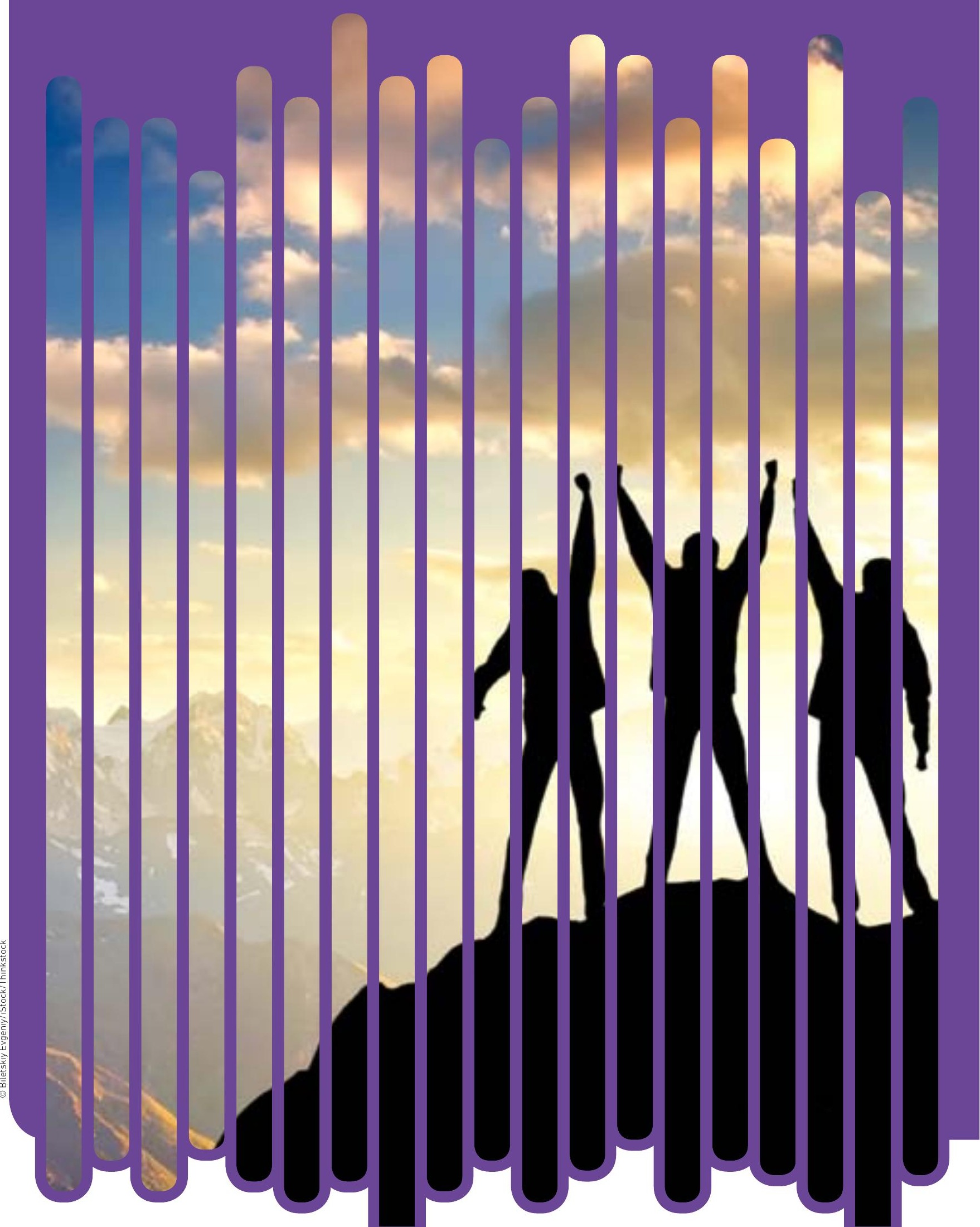




\section{HANDLUNGS- EMPFEHLUNGEN}

Allgemein: Förderung der Gemeinnützigkeit sollte eine Staatszielbestimmung werden

\section{Demokratie}

$\rightarrow$ Stärkere und verbindliche Nutzung der Komtenzen von CSOs und scheidungsprozessen, z.B. durch

$\Rightarrow$ angemessene Frist zur Begutachtung von Gesetzen, die es CSOs und BürgerInnen

erlaubt, Stellungnahmen abzugeben

$\Rightarrow$ Einführen von „Grünbüchern“ (Diskussionspapiere zu einem bestimmten Thema für die offentliche und wissenschaftliche Diskussion) und "Weilsbüchen (Zusammenfassungen der gesammelten Vorschlage) analog zur Praxis der $\Rightarrow$ En

Ensicklung und Ausbau neuer Partizipatiräten

\section{Vereinigungsfreiheit}

$\rightarrow$ Überarbeitung des $\$ 278$ a StGB mit dem Ziel zivilgesellschaftliches Engagement nicht zu gefährden und trotzdem Schutz vor kriminellen Vereinigungen zu gewährleisten

$\rightarrow$ Vereinsregister und damit die Registrierung der Vereine sollte, wie auch das Firmenbuch nicht beim Bundesminister

\section{Versammlungsfreiheit}

Stärkung des Wissens um Relevanz und Bedeutung der Versammlungsfreiheit im zivigesellschaftlichen Sektor, bei den Sicherheitsbehörden sowie in der gesamten Bevolkerung

$\rightarrow$ Explizite Anpassung des Versammlungsgesetzes an die Erfordernisse der EMRK

$\rightarrow$ Modifizierung von Gesetzen die zivilgesellschaftliches Engagement potenziell kriminalisieren: Z.B. Landfriedensbruch $\$ 274$ StGB $\rightarrow$ Zeitgerechte Beschwerdemechanismen be . möglichen Eilverfahren an Verwaltungsgerichten

$\rightarrow$ Risikoarme Beschwerdemechanismen für von unverhältnismäßigen $\mathrm{Ma} ß n$ hahmen ersammlungsteilnehmerInnen

$\rightarrow$ Gewährleistung der Identifikationsmöglichkeit von PolizistInnen durch an Uniformen sichtbare Erkennungsnummern, wie in Deutschland

$\rightarrow$ Systematische Verankerung des Prinzips der Deeskalation bei der Vorbereitung und Durchführung von Einsätzen bei Versammlungen.

$\rightarrow$ Reduktion von Haftungsrisiken für VeranstalerInnen von Versammlungen bei etwaigen Schäden, die ohne Vorsatz und ohne grobe Fahrlässigkeit entstanden sind. $\rightarrow$ Verbesserung der Förderbedingungen - keine Gegenrechnung von Förderungen und Einnahmen aus anderen Quellen (siehe auch Handlungsempfehlungen für die Bereitstellung von Dienstleistungen)

$\rightarrow$ Rechtliche/steuerliche Anreize für gemeinnützige Stiftungen

Förderung der Rahmenbedingungen für die Etablierung von Sozialen Banken

Herstellung der Verfassungskonformität in den jeweillgen Sammlungsgesetzen der Bundesländer, welche die Fördererwerbung durch CSOs derzeit noch potenziell unter Strafe

\section{Steuerrecht}

$\rightarrow$ Bestätigung der steuerlichen Begünstigung mittels enes individullen Rechtsakts (Festoder Frestellungsescintragung in eine Liste)

\section{Abschwächung der Forderung nach einer} wenis dem gleichen begünstigten Zweck dienen, Organisationen weitergegeben werden dorf

$\rightarrow$ Gleichstellung von gemeinnützigen und mildtätigen CSOs mit Sportvereinen bei der steueund sozialversicherungsfreien Auszahlung von Kostenersätzen an ehrenamtliche MitarbeiterInnen

$\rightarrow$ KESt-Befreiung für außerbetriebliches Vetmögen von Spenden sammelnden Organisationen und Stiftungen, das zur Finanzierung der gemeinnützigen Tätigkeit verwendet wird

$\rightarrow$ Ausweitung der Spendenbegünstigung auf alle anerkannten gemeinnützigen Zwecke, insbesondere die Bereiche Gesundheitsvorsorge, Bildung, Jugend, Tierschutz (auch ohne Tier-

heime), Kunst und Kultu

$\rightarrow$ Stärkere steuerliche Begünstigung der Errichtung von gemeinnützigen Stiftungen

Abrucken von der geforderten ausschließlichen Zweckerfüllung, um es insbesonder bereits bestehenden Privatstiftungen zu ig zu engagieren

öhung der maximalen Spendenabsetzbeträge und die Möglichkeit, diese auf mehrere u verteilen

$\rightarrow$ Rückerstattung der im nicht-unternehmerischen Bereich von CSOs anfallenden Vorsteue z.B. in Form einer Investitionsprämie
Beschäftigung und Freiwilligenarbeit

$\rightarrow$ Schaffung von Rechtssicherheit bei Zwischenformen von bezahlten und unbezahlten Tätiskeiten zivilgesellschaftlicher Organisationen $\Rightarrow$ Bereitstellung von Unfall- und Haftpflichtver-
sicherung für alle ehrenamtlichen Tätigkeiten

Bereitstellung von Dienstleistungen

$\rightarrow$ Gemeinnützigkeit in der Wettbewerbspolitik (auch in Bezug auf EU) stärken

$\rightarrow$ Planungssicherheit gewährleisten: Vereinbarung von mehrjährigen Rahmenverträgen mit Valorisierungsklauseln

$\rightarrow$ Qualitätsstandards für die Bereitstellung von Dienstleistungen im Auftrag des Staates definieren $=>$ Fokus der Kontrolle auf die Erfüllung der Qualitätsstandards (Ergebnisse) und weniger Vorgaben/Kontrollen bei der unmittelbaren Leistungserstellung

Vergabe nach dem Bestbieter- statt nach dem Billigstbieterprinzip. Wenn der Preis ausschlaggebend ist, damit Kriterien, damitVergleichbark gewährleistet ist

$\rightarrow$ Realistische budgetäre Vorgaben für die Ove

Zuständigkeiten bei mehreren Auftrag-/Fö

dergebern (zB.B.Bund/Land/Gemeinde/Sozidergebern (z.B. Bund/Land/Gen
versicherung etc.) besser klären

$\rightarrow$ Abrechnung vereinheitlichen und Kontrollen auf ein sinnvolles Maß reduzieren

$\rightarrow$ Aufrechtrechterhaltung der Vielfalt von CSO Unterstützungsmaßnahmen insbesondere für kleinere CSOs, z.B. Abfederung von ökonomischen Risiken, Unterstützung bei Einrei-

chungen

$\rightarrow$ Verhandlungsklima: CSOs als kompetente Partner/professionelle Anbieter anstatt als Bittsteller

\section{Transparenz bei der Vergabe von}

Leistungsverträgen und

Förderungen

$\rightarrow$ Erhöhung der Transparenz in Bezug auf $\Rightarrow$ aktuelle Information über die zur Verfügung stehenden Forder

die Höhe der Fördermittel und auf die Organidionen, die sie erhalten

$\Rightarrow$ die Begründung von $\mathrm{Zu}$-/Absagen
Wahrnehmbarkeit zivilgesellschaftlicher Wirkungen

$\rightarrow$ Zivilgesellschaftlichen und gemeinnützigen Themen in den öffentlich-rechtlichen Medien abseits von Spendenaufrufen mehr Raum CSOs stärker aufzeigen

$\rightarrow$ Stärkere Berücksichtigung der Rolle und der Funktionen von CSOs in der politischen Bildung

Anerkennung der Sprachrohr- und Korrektivfunktion (Advocacy) von CSOs bzw. einer vitalen Zivilgesellschaft.

Erstellung einheitlicher Statistiken über die Beschaffenheit und die Wirkungen des dritten 
APPENDIX

\& LITERATUR

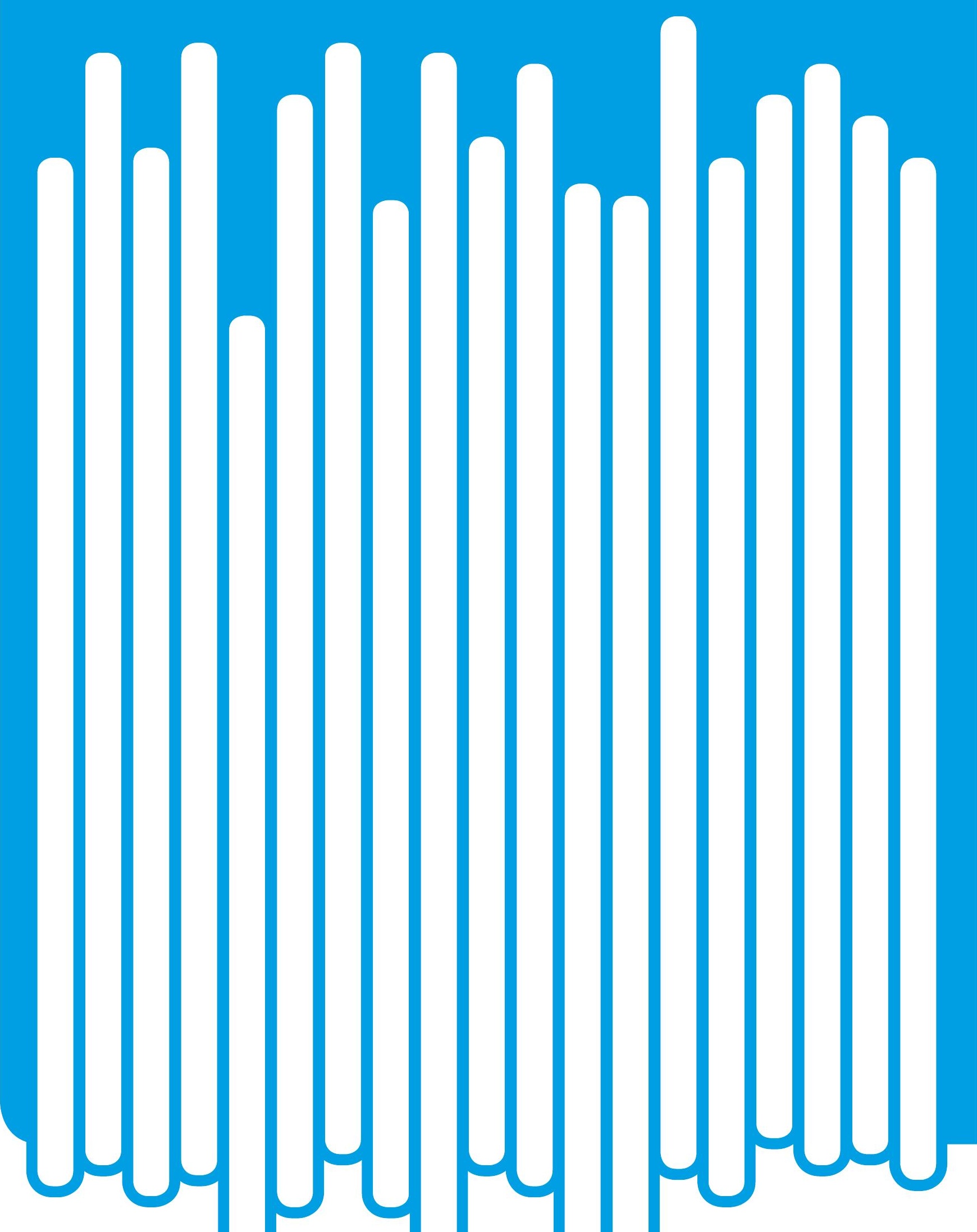




\section{NATIONAL ADVISORY GROUP}

Robert Dempfer

4. Gerald Fitz

5. Gabriele Gerbasits

6. Katharina Meichenitsch

7. Filip Radunovic

8. Hanna Simons

9. Philipp Sonderegger

Io. Barbara Weber

I. Wilhelm Zwirner

\section{INTERVIEW-PARTNERINNEN}

\section{CSO-VertreterIn}

CSO-VertreterIn

3. CSO-VertreterIn

$\begin{array}{ll}\text { 4. } & \text { CSO-VertreterIn } \\ 5 . \quad \text { CSO-VertreterIn }\end{array}$

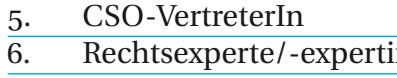

7. Rechtsexperte/-experti

9. Rechtsexperte/-expertin

$\begin{array}{ll}\text { 9. } & \text { Rechtsexperte/-exp } \\ \text { I0. } & \text { CSO-VertreterIn }\end{array}$

1. CSO-VertreterIn

12. Rechtsexperte/-expertin

14. Rechtsexperte/-expertin

15. CSO-VertreterIn

I6. Rechtsexperte/-expertin

I7. CSO-VertreterIn

17. CSO-VertreterIn

I9. CSO-VertreterIn

2I. Rechtsexperte/-expertin

2. Rechtsexperte/-expertin

23. Rechtsexperte/-expertin

24. Rechtsexperte/-expertin

\section{THEMENSCHWERPUNKT}

Forderungen/Vergabe, Freiwillige Orderungen/Vergabe, Freiwillige Oorderungen/Vergabe, Freiwillige Förderungen/Vergabe, Freiwillige Forderungen/Vergabe, Freiwillige Menschen-/BürgerInnenrec Vergaberecht

Freiwillige, Förderungen/Vergabe orderungen/Vergabe

Menschen-/BürgerInnenrecht Steuerrecht Senschen-/BürgerInnenrech Steuerrecht Menschen- / BürgerInnenrecht Menschen- / BürgerInnenrecht Menschen- /BürgerInnenrecht Menschen- / BürgerInnenrech Steuerrecht Steuerrecht Steuerrecht
Steuerrecht
ACHATZ, MARKUS (2006): Verfassungsrechtliche und europarechtliche Aspekte der BesteueRecht, WiR - Studiengesellschaft f. Wirtscha u. (Hrsg.): Das Recht der Non-Profit-Organ sationen, Wien: Linde, 49-96.

ACHATZ, Markus/Oberleitner, Siegfried (2013): Besteuerung und Rechnungslegung der Vere

OLFF, FRANK (2005): Wirtschaft und Zivilgesellschaft. Theoretische und empirische Perspektiven. I., Wiesbaden: VS Verl. für Soziawiss.

Anastasiadis, Maria/Schmid, Tom/EssL, GÜNTER/RIESENFELDER, ANDREAS/ WeTZel, Petra (2003): Der Dritte Sektor in Wien - Zukunftsmarkt der Beschäftigung. unveröff. Projektbericht. Wien.

R, HELMUT K. (2013): Entwicklungen der internationalen Zivilgesellschaft. In: Sims (Hrso): Handbuch der Nonprofit-Organisa(Hog.). Hand Stuttgart: Schäffer-Poeschel, 77-89.

ANSASTASiadis, Maria/Mayrhofer, Marlene/ Sснмid, Том (2005): Finanzielle Situation und Strategien. Toolbox - Weiterentwicklung von Organisationen im Dritten Sektor. Wien: 20-66, http://www.lrsocialresearch.at/files/ Toolbox_fuer_den_Dritter_Sektor.pdf.

ARMINGEON, KLAUS (2012): Interessensgruppen und Interessensvermittlung: Internationale Gemeinsamkeiten und österreichisch Wineroither chische Demokratie im Vergleich. I., neue Ausg Aufl., Baden-Baden: Nomos, 223-248.

BIRKhÖLZER, KARL/KLeIN, ANSGAR/PRILLER, ECKARD/ZIMMER, ANNETTE (2005): Dritter Sektor - Drittes System. Theorie, Funktion wandel und zivilgesellschaftliche Perspektiven. I., Wiesbaden: VS, Verl. für Sozialwis

BÜRO FÜR ZUKUNFTSFRAGEN VORARLBERG (20 Io): Der BürgerInnen-Rat: Eine Informations- und Arbeitsmappe.

DiER, PEIn (2012). Die territoriale Dimension der österreichischen Demokratie in vergleichender Perspektive. In: Helms, Ludger/Winger, David M. (Hrsg.): Die neue Ausg Aufl., Baden-Baden: Nomos, I7 I-

I93.
CAMPBELL, David FJ. (2012): Die österreichische Demokratiequalität in Perspektive. In: Helms, Ludger/Wineroither, David M. (Hrsg): Die österreichische Demokratie im Vergleich. neue Ausg Aufl., Baden-Baden: Nomos, 293315.

Campbell, David FJ./Barth, Thorsten D. (2009): Wie können Demokratie und Demokratiequalität gemessen werden?: Modelle, Demokratie-Indices und Länderbeispiele im globalen Vergleich. In: SWS-Rundschau, 49 209-233
Chambers, Simone/Kopstein, Jeffrey (200 I): Bad Civil Society. In: Political Theory 29, 837-865

Crouch, Collin (2013): Post-Democracy. The challenge for Europe. In: Queries. The European $P$.

Dathe, DietMar/HohendanNer, Christian/ PrILLER, ECKHARD (2009): Wenig Licht, vie Schatten - der Dritte Sektor als arbeitsmark politisches Experimentierfeld. In: WZBrief Abreit.

DEMOCRACY BAROMETER (2013):Austria 1990-2007. IMMEL, NIKOLAUS (20I2): Sozialwirtschaft unt Prekarisierungsdruck. In: WISO (Wirtschaftsund sozialpolitische Zeitschrift) Publikation des Instituts fur Sozial- und Wirtschatswissenschaften, 2012

EDER, EVA MARIA (2009): Freiwilligenarbeit unte arbeitsrechtlichen Gesichtspunkten. In: BMASK (Hrsg.): Freiwilliges Engagement BMASK, I3-I6.

European COMmission (2013): :Standard Eurobarometer 80 Autumn 2013: Pulic Opinon in the European Union.

VS (20I I): European Values Study 2008.: Integrated Dataset (EVS 2008) ZA480o Data file

Fioramonti, LORENZO/THÜMLER, EKKEHARD (2013): Citizens vs. Markets. How Civil Society is Rethinking the Economy in a Tim of Crisis. New York: Routledge.

FREEDOM House (20I4A): Freedom in the Press 2013 Austria.

REEDOM HOUSE (20I4B): Freedom in the World 2013 Austria.

REEDOM House (20I4C): Freedom in the World: Country Ratings 1972-2013.

RIEDRICH, PETER/NALTSCHOTZ, ANITA/NAM, CHANG WOON/PARSCHE, RÜDIGER/WELLISCH, DieTMAR (2005): Die Besteuerung gemeinnütziger Organisationen im internationalen Vergleich. Forschungsvorhaben de Bundesministeriums der Finanzen. München: Institut für Wirtschaftsforschung a der Universität München.

FA (2013A): Spendenbericht 2013. Austria, Fundraising Verband. Wien

VA (2013B): Vorschlag für ein Regierungsprogramm 2013 bis 2018, Kapitel Steuern und Stiftungstandorts Österreich. Austria, Fundraising Verband. Wien. Bachmann Susanne/Baumgartee, Gethard/ Feik, Rudolf/Giese, Karim/Jahnel, Dietmar/ Lienbacher, Georg (Hrsg): Besonderers Verwaltungsrecht. 8 Aufl., Wien: Springer, 7 I-95

HAIDER, ASTRID/SCHNEIDER, ULRIKe (2009): Das Verhältnis von Freiwilligenarbeit und bezahlter Arbeit in Nonprofit Organistationen. In: BMASK (Hrsg.): Freiwilliges Engagement in Österreich. I. Freiwilligenbericht, Wien: BMASK, I48-I6I 
Heidenbauer, Sabine (20I I): Charity Crossing Borders. The Fundamental Freedoms' Influence on Charity and Donor Taxation in Kluwer Law International.

Heimgartner, ARno (2009): Strukturelle Rahmenbedingungen für freiwilliges Engagement. In: BMASK (Hrsg.): Freiwilliges Engagement in Österreich. I. Freiwilligenberich Wien: BMASK, I62-I75.

HELMS, LUDGER/WINEROITHER, DAVID M. (2012) Nationalrat, Bundesregierung und Bundespräsident: Die gouvernementale Arena im internationalen Vergleich. In: Helms, Ludger/ Wineroither, David M. (Hrsg.): Die österreichische Demokratie im Vergleich. I., neue Ausg Aufl., Baden-Baden: Nomos, I 47-I70.

HÖHNE, THOMAS/JöCHL, GERHARD/LUMMERSTORFER, ANDREAS (2009): Das Recht der Vereine: Privatrechtliche, öffentlich-recht-
liche und steuerrechtliche Aspekte 3. Auflage liche und steuerrechtliche Asper

IDEA Voter TURNOUT DATABASE (20I4): Wähle beteiligung an nationalen und EU Parla( from http://epp.eurostat.ec.europa.eu.

IGO, INIERESSENVERTRETUNG GEMEINNÜTZIGER ORGANISATIONEN (2013): Ref Ergebnisse einer Online-Umfrage unter Leitungsverantwotlichen gemeinnütziger Organisationen. Wien.

Klein, ANsGar/Rohde, Markus (2003): Konturen der Zivilgesellschaft - Zur Profilierun eines Begriffs. Editorial zum Themenheft. In Forschungsjournal Neue Soziale Bewegungen, I6, 2-5.

Krabb, Philipp (20 I I): Probleme der Demokratiequalitat Osterreichs. Eine qualitative Analyse. Diplomarbeit an der Universität Wien.

LAUTH, HANS-JOACHIM (2004): Demokratie und Demokratiemessung: Eine konzeptionelle Grundlegung fưr den interkulturellen Vergleich. I. Aufl, Wiesbaden: VS Verl. für Sozialwissenschaften

LIEBIG, REINHARD/KARLA, Esther (2003): Entwicklungsfaktoren und -dynamiken des Dritten Sektors als Arbeitsmarkt. Dortmun Universität Dortmund.

LitTich, Edith/SCHObER, Christian (2013): Finanzierung von NPOs. In: Simsa, Ruth/ Meyer, Much der Nonprofit-Orstoph (Hrsg.) Handbuch deruk Nonprofi-Organisation Schäffer-Poeschel, 289-3I2.

Marshall, Monty G./GurR, Ted Robert (20I I): Polity IV Project: Country Report 2010 Austria.

MARTERBAUER, MARKus (20I I): Zahlen Bitte! Die Kosten der Krise tragen wir alle. Wien: Deutike.

MatZInGER, Johannes (2012): Politische Partizipation von MigrantInnen in Österreich. Eine hintergrunds im European Values Study 2008 Diplomarbeit an der Universität Wien.
Meyer, Michael/Simsa, Ruth (20I3): Entwicklungsperspektiven des Nonprofit-Sektors. In Simsa, Ruth/Meyer, Michael/Badelt Christoph (Hrsg.): Handbuch der Nonprofit-Organisation. Strukturen und Management. 5. Aufl., Stuttgart: Schäffer-Poeschel, 509-525.

More-Hollerweger, Eva/Hora, Katrin (2014): Rahmenbedingungen für freiwilliges Engagement in Österreich. Ergebnisse einer Organisationsbefragung. Wien: NPO \& SE Kompetenzzentrum der WirtschaftsuniversitätWien.

Neumayr, Michaela (2010): Resource dependence. Der Einfluss öffentlicher Finanzierungsformen auf die Funktionen von Nonprofit Organisationen in Osterreich. Wien: Doctoral Thesis Wirtschaftsuniversität Wien.

OBERHUBER, FLORIAN/BREITENFELDER, URSULA/WITTINGER, DANIELA (2012): Die Einbindung der Zivilgesellschaft in das Reformprogramm Europa 2020 in

OECD (2010): Government Social Spending.

Pelinka, Anton (201 I): Was ist demokratische Quact. Ostrum Barbara/Duffek, Karl A. (Hrsg): Die Qualitat der Demokratie, Wien, 55-74.

PENNERSTORFER, Astrid/SCHNEIDER, ULRIKe/ BADELT, CHRISTOPH (20I3): Der Nonprofit Sektor in Österreich. In: Simsa, Ruth/Meye Michael/Badelt, Christoph (Hrso): Handbuch der Nonprofit Organisationen. Strukturen und Management. 5. Aufl., Stuttgart: Schäffer-Poeschel Verlag, 55-75.

PEPIN, JoHN (2005): Venture capitalists and entrepreneurs become venture philanthropists. In: International Journal of Nonprofit \& Voluntary Sector Marketing, I0, I65-I73.

PLASSER, Fritz/SEEBER, GILG (2012): Politische Kultur und Demokratiebewusstsein in der Zweiten Republik im internationalen Vergleich. In: Helms, Ludger/Wineroither, David M. (Hrsg.): Die osterreichische Demokratie im Vergleich. I., neue Ausg Aufl., BadenBaden: Nomos, 269-292.

( (2009): Beteiligung am freiwilligen Engagefür Arbeit, Sozioich. In: Bundesministerium (r) eich. I. Freiwilligenbericht, Wien: BMASK, 49-73. (20I I): Kriminelle Organisation (S278 StGB). Eine dogmatische Evaluierung des Tatbestandes im Auftrag des Bundesministeriums für Justiz. Wien, unveröffentlichter Projektbericht.

Rosenberger, SIEgLINDE/SEeber, GiLg (2007) Volksbegehren in Österreich. In: Der Bürger im Staat.
SALAmon, Lester M./ Anheier, Helmut K. (1992): In Search of the Nonprofit Sector I: The Ques

AnNa/Millner, Reinhard/ MEYER, MiCHAEL (2010): Die Rolle der Gemeinnüzigkeit in Osterreichischen StifWen. Working Paper der Abteilung für sität Wien. Wien: Abteilung für Nonprofit Management Witelung

SCHNEIDER, UlRiKe/HAIDER, Astrid (2009): Nonprofit Organisationen in Osterreich 2006. In: Forschungsbericht des Instituts für Sozialpolitik, WU Wien, FBor / 2009

SCHNEIDER, ULRIKE/OSTERLE, AUGUST/ SCHOBER, DORIS/SCHOBER, CHRISTIAN (2006): Die Kosten der Plege in Osterreich. Ausgabenstrukturen und Finanzierung. Forschungsbericht des Instituts fưr Sozialpolitik, Wirtschaftsuniversitat Wien. Wien: Institut

für Sozialpolitik, Wirsciculs Doris/LiNTSCHINGER, LISA (20II): Die unterschiedlichen Finanzierungsquellen von NPOs. Wien WU Wien NPO-Kompetenzzentrum, unveröffentlichter Projektbericht.

SCHOBER, Christian/More-Hollerweger, Eva/Rauscher, Olivia (20I2): SROI-Anayse für die Feuerwehren in Oberösterreich. Projektbericht. Wien.

SCHOBER, CHRISTIAN/SCHOBER, DoRis (2004): Personalmanagement, Arbeitszufriedenheit und Motivation in Organisationen der Altenpflege und -betreuung. Wien Dissertation Wirtschaftsuniversität

SCHÜtZ, ROBERT/RUNTE, JULIA (20I3): Reform des Gemeinnützigkeitsrechts - Bundesrat stimmt Ehrenamtsstarkungsgesetz zu. compact spezial, Stiftungen und gemeinnützige Organisationen. Hamburg: Esche-Schümann-Commichau.

SIMSA, RUTH (2013A): Gesellschaftliche Restgröße oder treibende Kraft? Soziologische Perspektiven auf NPOs. In: Simsa, Ruth/ Meyer, Michael/Badelt, Christoph (Hrsg.): Handbuch der Nonprofit-Organisation. Strukturen und Management. 5. Aun., Stut gart: Schäffer \& Poeschel, I25-I 45

SIMSA, RUTH (20I3B): Protest ohne Organisation? in. Neue Sozlale Bewegungen 4

A (2013): Dingedingen us NO Wirtschafts- und Sozialpolitische Zetschrift des IWS, 2013, I63-I68.

STATISTIK AuSTRIA (20I2): Statistisches Jahrbuch Österreichs 2012. Verlag Österreich GmbH.

UCAKAR, KARL/GSCHIEGL, STEFAN (2009): Das politische System Österreichs und die EU. Wien: Facultas.wuv.

Verba, Sidney/Lehman SCHLozman, Kay / BRADY, HENRY E. (1995): Voice and Equality: Civic Voluntarism in American Politics. Cambridge, MA: Harvard University Press.
WALter, Florian (2012): Von Teilhabe ausgechlossen? Soziale Ungleichheit und politische Partizipation in Österreich. In Kurswechsel $40-48$

Wineroither, DAVID M. / KitsChelt, Herbert (2012): Die Entwicklung des Parteienwettbewerbs in Österreichs im internationalen Vergleich. In: Helms, Ludger/Wineroither, David M. (Hrsg.): Die österreichische Demokratie m Vergleich. I., neue Ausg Aufl., BadenBaden: Nomos, 193-222.

WINHELLER, STEFAN (2008): Aktuelle Entwicklungen im Gemeinnützigkeitsrecht 2007. In: Deutsche Steuerzeitung, 28I-291.

YAN, WENLI/DENISON, DWIGHT V./BUTLER, J.S (2009): Revenue Structure and Nonprofit Borrowing. In: Public Fhance Review, 37 47-67.

ZENGERLING, MatTHIAS (2008): Änderungen im Gemeinnützigkeitsrecht im Zuge des Gesetzes zur Stärkung des bürgerschaftlchen Engagements. Seminararbeit im

lungen im Recht der Abgabenordnung. Jena Friedrich-Schiller-Universität Jena.

Zimmer, ANNETte/Priller, ECKhard (2007): Gemeinnützige Organisation Sektor-Forschung 2, Wiesbaden:VSVerlag für Sozialwissenschaften. 


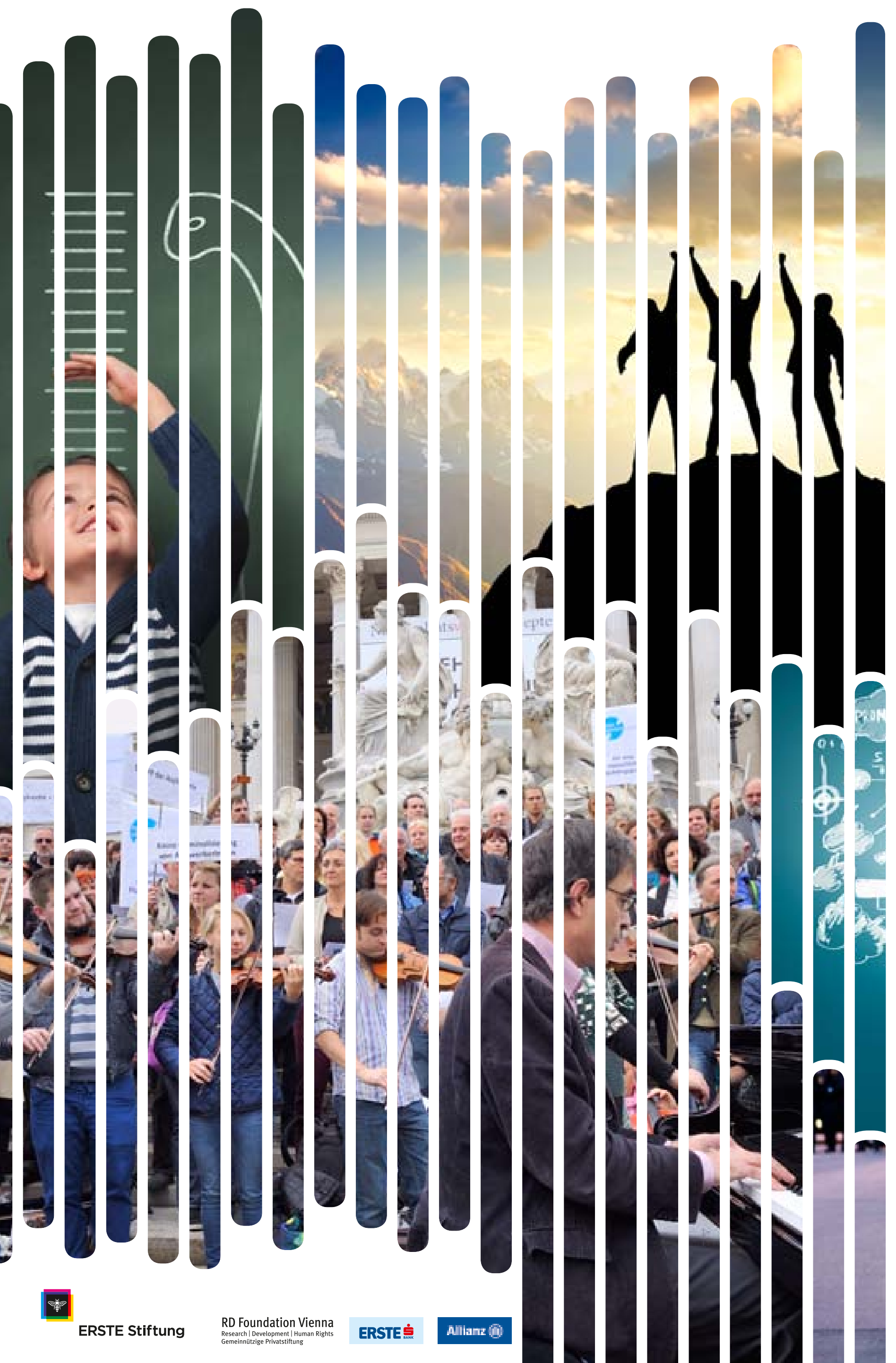

